\title{
Homomorphic signatures with sublinear public keys via asymmetric programmable hash functions
}

\author{
Dari॰ Catalan॰ ${ }^{1}$ - Dariø Fiøre ${ }^{2}$ - Luca Nizzard $\bullet^{3}$
}

\begin{abstract}
We introduce the notion of asymmetric programmable hash functions (APHFs, for short), which adapts Programmable hash functions, introduced by Hofheinz and Kiltz (Crypto 2008, Springer, 2008), with two main differences. First, an APHF works over bilinear groups, and it is asymmetric in the sense that, while only secretly computable, it admits an isomorphic copy which is publicly computable. Second, in addition to the usual programmability, APHFs may have an alternative property that we call programmable pseudorandomness. In a nutshell, this property states that it is possible to embed a pseudorandom value as part of the function's output, akin to a random oracle. In spite of the apparent limitation of being only secretly computable, APHFs turn out to be surprisingly powerful objects. We show that they can be used to generically implement both regular and linearly-homomorphic signature schemes in a simple and elegant way. More importantly, when instantiating these generic construc-tions with our concrete realizations of APHFs, we obtain: (1) the first linearly-homomorphic signature (in the standard model) whose public key is sub-linear in both the dataset size and the dimension of the signed vectors; (2) short signatures (in the standard model) whose public key is shorter than those by Hofheinz-Jager-Kiltz (Asiacrypt 2011, Springer, 2011) and essentially the same as those by Yamada et al. (CT-RSA 2012, Springer, 2012).
\end{abstract}

Luca Nizzardo

luca.nizzardo@imdea.org

Dario Catalano

catalano@dmi.unict.it

Dario Fiore

dario.fiore@imdea.org

1 Dipartimento di Matematica e Informatica, Università di Catania, Catania, Italy

2 IMDEA Software Institute, Madrid, Spain

3 IMDEA Software Institute and Universidad Politécnica de Madrid, Madrid, Spain 


\section{Introduction}

Programmable hash functions Programmable hash functions (PHFs) were introduced by Hofheinz and Kiltz [30] as an information theoretic tool to "mimic" the behavior of a random oracle in finite groups. In a nutshell, a PHF $\mathrm{H}$ is an efficiently computable function that maps suitable inputs (e.g., binary strings) into a group $\mathbb{G}$, and can be generated in two different, indistinguishable, ways. In the standard modality, $\mathrm{H}$ hashes inputs $X$ into group elements $H(X) \in \mathbb{G}$. When generated in trapdoor mode, a trapdoor allows one to express every output in terms of two (user-specified) elements $g, h \in \mathbb{G}$, i.e., one can compute two integers $a_{X}, b_{X}$ such that $\mathrm{H}(X)=g^{a_{X}} h^{b_{X}}$. Finally, $\mathrm{H}$ is programmable in the sense that it is possible to program the behavior of $H$ so that its outputs contain (or not) $g$ with a certain probability. More precisely, $\mathrm{H}$ is said $(m, n)$-programmable if for all disjoint sets of inputs $\left\{X_{1}, \ldots, X_{m}\right\}$ and $\left\{Z_{1}, \ldots, Z_{n}\right\}$, the joint probability that $\forall i, a_{X_{i}}=0$ and $\forall j, a_{Z} \neq 0$ is significant (e.g., $1 /$ poly $(\lambda)$ ). Programmability turns out to be particularly useful in several security proofs. For instance, consider a security proof where a signature on $H(X)$ can be simulated as long as $a_{X}=0$ (i.e., $g$ does not appear) while a forgery on $H(Z)$ can be successfully used if $a_{Z} \neq 0$ (i.e., $g$ does appear). Then one could rely on an $(m, 1)-$ programmability of $\mathrm{H}$ to "hope" that

all the queried messages $X_{1}, \ldots, X_{m}$ are simulatable, i.e., $\forall i, a_{X_{i}}=0$, while the forgery message $Z$ is not, i.e., $a_{Z} \neq 0$. PHFs essentially provide a nice abstraction of the so-called partitioning technique used in many cryptographic proofs.

\subsection{Our contribution}

Asymmetric programmable hash functions We introduce the notion of asymmetric programmable hash functions (APHFs) which modifies the original notion of PHFs [30] in two main ways. First, an APHF $\mathrm{H}$ maps inputs into a bilinear group $\mathbb{G}$ and is only secretly computable. At the same time, an isomorphic copy of $\mathrm{H}$ can be publicly computed in the target group $\mathbb{G}_{T}$, i.e., anyone can compute $e(\mathrm{H}(X), g) .{ }^{1}$ Second, when generated in trapdoor mode, for two given group elements $g, h \in \mathbb{G}$ such that $h=g^{z}$, the trapdoor allows one to write every $H(X)$ as $g^{c_{X}(z)}$ for a degree- $d$ polynomial $c_{X}(z)$.

We define two main programmability properties of APHFs. The first one is an adaptation of the original programmability notion, and it says that $\mathrm{H}$ is $(m, n, d)$-programmable if it is $(m, n)$-programmable as before except that, instead of looking at the probability that $a_{X}=0$, one now looks at whether $c_{X, 0}=0$, where $c_{X, 0}$ is the coefficient of the degree- 0 term of the polynomial $c_{X}(\cdot)$ obtained using the trapdoor. ${ }^{2}$ The second programmability property is new and is called programmable pseudorandomness. Roughly speaking, programmable pseudorandomness says that one can program $\mathrm{H}$ so that the values $g^{c X, 0}$ look random to any polynomially-bounded adversary who observes the public hash key and the outputs of $\mathrm{H}$ on a set of adaptively chosen inputs. This functionality turns out to be useful in security proofs where one needs to cancel some random values for simulation purposes (we explain this in slightly more detail later in the introduction). In other words, programmable pseudorandom-

1 Because of such asymmetric behavior we call these functions "asymmetric".

${ }^{2}$ For $d=1$, this is basically the same form of programmability of [30]. 
ness provides another random-oracle-like property for standard model hash functions, that is to "hide" a PRF inside the hash function. This is crucial in our security proofs, and we believe it can have further applications.

Applications In principle, secretly computable PHFs seem less versatile than regular PHFs. In this work, however, we show that, for applications such as digital signatures, APHFs turn out to be more powerful than their publicly computable counterparts. Specifically, we show how to use APHFs to realize both regular and linearly-homomorphic signatures secure in the standard model. Next, we show efficient realizations of APHFs that, when plugged in our generic constructions, yield new and existing schemes that improve the state-of-the-art in the following way. First, we obtain the first linearly homomorphic signature scheme, secure in the standard model, achieving a public key which is sub-linear in both the dataset size and the dimension of the signed vectors. Second, we obtain regular signature schemes, matching the efficiency of the ones in [39], thus providing the shortest signatures in the standard model with a public key shorter than in [32].

In the following we elaborate more on these solutions.

Linearly-homomorphic signatures with short public key in the standard model Imagine a user Alice stores one or more datasets $D_{1}, D_{2}, \ldots, D_{\ell}$ on a cloud server. Imagine also that some other user, Bob, is allowed to perform queries over Alice's datasets, i.e., to compute one or more functions $F_{1}, \ldots, F_{m}$ over any $D_{i}$. The crucial requirement here is that Bob wants to be ensured about the correctness of the computation's results $F_{j}\left(D_{i}\right)$, even if the server is not trusted. An obvious way to do this (reliably) is to ask Alice to sign all her data $D_{i}=m_{1}^{(i)}, \ldots, m_{N}^{(i)}$. Later, Bob can check the validity of the computation by (1) downloading the full dataset locally, (2) checking all the signatures and (3) redoing the computation from scratch. Efficiency-wise, this solution is clearly undesirable in terms of bandwidth, storage (Bob has to download and store potentially large amount of data) and computation (Bob has to recompute everything on his own).

A much better solution comes from the notion of homomorphic signatures [11]. These allow to overcome the first issue (bandwidth) in a very elegant way. Using such a scheme, Alice can sign $m_{1}, \ldots, m_{N}$, thus producing signatures $\sigma_{1}, \ldots, \sigma_{N}$, which can be verified exactly as ordinary signatures. In addition, the homomorphic property provides the extra feature that, given $\sigma_{1}, \ldots, \sigma_{N}$ and some function $F: \mathcal{M}^{N} \rightarrow \mathcal{M}$, one can compute a signature $\sigma_{F, y}$ on the value $y=F\left(m_{1}, \ldots, m_{N}\right)$ without knowledge of the secret signing key sk. In other words, for a set of signed messages and any function $F$, it is possible to provide $y=F\left(m_{1}, \ldots, m_{N}\right)$ along with a signature $\sigma_{F, y}$ vouching for the correctness of $y$. The security notion of homomorphic signatures guarantees that creating a signature $\sigma_{F, y^{*}}$ for a $y^{*} \neq F\left(m_{1}, \ldots, m_{N}\right)$ is computationally hard, unless one knows sk.

To solve the second issue and allow Bob to verify efficiently such signatures (i.e., by spending less time than that required to compute $F$ ), one can use homomorphic signatures with efficient verification, a notion recently introduced in [20]. The notion of homomorphic signature was first introduced by Johnson et al. [33]. Since then several schemes have been proposed. The first schemes were homomorphic only for linear functions over vector spaces $[3-5,12$, $14,16-18,22,24,35]$ and have nice applications to network coding and proofs of retrievability. More recent works proposed realizations that can support more expressive functionalities such as polynomials $[11,20]$ or general circuits of bounded polynomial depth $[15,26]$.

Despite the significant research work in the area, it is striking that all the existing homomorphic signature schemes that are proven secure in the standard model $[3-5,15-17,20,22,26,35]$ suffer from a public key that is at least linear in the size $N$ of the signed datasets. On one hand, the cost of storing such large public key can be, in principle, amortized since the key 
can be re-used for multiple datasets. On the other hand, this limitation still represents a challenging open question from both a theoretical and a practical point of view. From a practical perspective, a linear public key might be simply unaffordable by a user Bob who has limited storage capacity. From a theoretical point of view, considered the state-of-the-art, it seems unclear whether achieving a standard-model scheme with a key of length $o(N)$ is possible at all. Technically speaking, indeed, all these schemes in the standard model somehow rely on a public key as large as one dataset for simulation purposes. This essentially hints that any solution for this problem would require a novel proof strategy.

Our contribution We solve the above open problem by proposing the first standard-model homomorphic signature scheme that achieves a public key whose size is sub-linear in the maximal size $N$ of the supported datasets; moreover, our scheme is context-hiding secure. Slightly more in detail, we show how to use APHFs in a generic fashion to construct a linearly-homomorphic signature scheme based on bilinear maps that can sign datasets, each consisting of up to $N$ vectors of dimension $T$. The public key of our scheme mainly consists of the public hash keys of two APHFs. By instantiating these using (one of) our concrete realizations we obtain a linearly-homomorphic signature with a public key of length $O(\sqrt{N}+\sqrt{T})$. We stress that ours is also the first linearly-homomorphic scheme where the public key is sub-linear in the dimension $T$ of the signed vectors. Concretely, if one considers applications with datasets of 1 million of elements and a security parameter of 128 bits, previous solutions (e.g., $[4,17])$ require a public key of at least $32 \mathrm{MB}$, whereas our solution simply works with a public key below $100 \mathrm{~KB}$.

On the power of secretly-computable PHFs The main technical idea underlying this result is a new proof technique that builds on asymmetric hash functions with programmable pseudorandomness. We illustrate the technique via a toy example inspired by our linearlyhomomorphic signature scheme. The scheme works over asymmetric bilinear groups $\mathbb{G}_{1}, \mathbb{G}_{2}$, and with an APHF $\mathrm{H}:[N] \rightarrow \mathbb{G}_{1}$ that has programmable pseudorandomness w.r.t. $d=1$. To sign a random message $M \in \mathbb{G}_{1}$ w.r.t. a label $\tau$, one creates the signature

$$
S=(\mathrm{H}(\tau) \cdot M)^{1 / z}
$$

where $z$ is the secret key. The signature is linearly-homomorphic- $S_{1} S_{2}=\left(\mathrm{H}\left(\tau_{1}\right) \mathrm{H}\left(\tau_{2}\right) M\right)^{1 / z}$, for $M=M_{1} M_{2}$ - and it can be efficiently checked using a pairing $-e\left(S, g_{2}^{z}\right)=$ $\prod_{i} e\left(\mathrm{H}\left(\tau_{i}\right), g_{2}\right) e\left(M, g_{2}\right)$ - and by relying on that $e\left(\mathrm{H}(\cdot), g_{2}\right)$ is publicly computable.

The first interesting thing to note is that having $\mathrm{H}$ secretly computable is necessary: if $\mathrm{H}$ is public the scheme could be easily broken, e.g., choose $M^{*}=H(\tau)^{-1}$. Let us now show how to prove its security assuming that we want to do a reduction to the following assumption: given $g_{1}, g_{2}, g_{2}^{z}$, the challenge is to compute $W^{1 / z} \in \mathbb{G}_{1}$ for $W \neq 1$ of adversarial choice. Missing $g_{1}^{z}$ seems to make hard the simulation of signatures since $M, S \in \mathbb{G}_{1}$. However, we can use the trapdoor generation of $\mathrm{H}$ for $d=1$ (that for asymmetric pairings takes $g_{1}, h_{1}=g_{1}^{y_{1}}, g_{2}, h_{2}=g_{2}^{y_{2}}$ and allows to express $\left.\mathrm{H}(X)=g_{1}^{c_{X}\left(y_{1}, y_{2}\right)}\right)$, by plugging $h_{1}=1, h_{2}=g_{2}^{z}$. This allows to write every output as $H(\tau)=g_{1}^{c_{\tau}(z)}=g_{1}^{c_{\tau, 0}+c_{\tau, 1} z}$. Every signing query with label $\tau$ is simulated by setting $M_{\tau}=g_{1}{ }^{-c_{\tau, 0}}$ and $S_{\tau}=\left(g_{1}^{c_{\tau, 1}}\right)$. The signature is correctly distributed since (1) $S_{\tau}=\left(\mathrm{H}(\tau) \cdot M_{\tau}\right)^{1 / z}$, and (2) $M_{\tau}$ looks random thanks to the programmable pseudorandomness of $\mathrm{H}$. To conclude the proof, assume that the adversary comes up with a forgery $M^{*}, S^{*}$ for label $\tau^{*}$ such that $\tau^{*}$ was already queried, and let $\hat{S}, \hat{M}$ be the values in the simulation of the signing query for $\tau^{*}$. Now, $\hat{S}=\left(\mathrm{H}\left(\tau^{*}\right)\right.$. $\hat{M})^{1 / z}$ holds by correctness, while $S^{*}=\left(\mathrm{H}\left(\tau^{*}\right) \cdot M^{*}\right)^{1 / z}$ holds for $M^{*} \neq \hat{M}$ by definition of forgery. Then $\left(M^{*} / \hat{M}, S^{*} / \hat{S}\right)$ is clearly a solution to the above assumption. This essentially shows that we can sign as many $M$ 's as the number of $\tau$ 's, that is $N$. And by using our 
construction $\mathrm{H}=\mathrm{H}_{\text {sqrt }}$ this is achievable with a key of length $O(\sqrt{N})$. Let us stress that the above one is an incomplete proof sketch, that we give only to illustrate the core ideas of using programmable pseudorandomness. Moreover, note that the one presented above is only one of the possible cases of a forgery, but we think that it is the most interesting one to be considered in our example. We defer the reader to Sect. 4 for a precise description of our signature scheme and its security proof.

Short signatures from bilinear maps in the standard model Hofheinz and Kiltz [30] proposed efficient realizations of PHFs, and showed how to use them to obtain black-box proofs of several cryptographic primitives. Among these applications, they use PHFs to build generic, standard-model, signature schemes from the Strong RSA problem and the Strong $q$-Diffie-Hellman problem. Somewhat interestingly, these schemes (in particular the ones over bilinear groups) can enjoy very short signatures. The remarkable contribution of the generic construction in [30] is that signatures can be made short by reducing the size $\rho$ of the randomness used (and included) in the signature so that $\rho$ can go beyond the birthday bound. Precisely, by using an $(m, 1)$-programmable hash function, $m$ can control the size of the randomness so that the larger is $m$, the smaller is the randomness. However, although this would call for $(m, 1)$-PHFs with a large $m$, the original work [30] described PHFs realizations that are only $(2,1)$-programmable. ${ }^{3}$

Later, Hofheinz et al. [32] showed constructions of ( $m, 1)$-PHFs for any $m \geq 1$. By choosing a larger $m$, these new PHFs realizations yield the shortest known signatures in the standard model. On the negative side, however, this also induces much larger public keys. For instance, to obtain a signature of 302 bits from bilinear maps, they need a public key of more than $8 \mathrm{MB}$. The reason of such inefficiency is that their realizations of (deterministic) $(m, 1)$ PHFs have keys of length $O\left(m^{2} \ell\right)$, where $\ell$ is the bit size of the inputs. In a subsequent work, Yamada et al. [39] improved on this aspect by proposing a signature scheme with a public key of length $O(m \sqrt{\ell})$. Their solution followed a different approach: instead of relying on $(m, 1)$-PHFs they obtained the signature by applying the Naor's transformation [8] to a new identity-based key encapsulation mechanism (IBKEM).

Our results Our results are mainly two. First, we revisit the generic signature constructions of $[30,32]$ in order to work with $(m, 1, d)$-APHFs. Our generic construction is very similar to that in [30,32], and, as such, it inherits the same property: the larger is $m$, the shorter can be the randomness.

Second we show the construction of an APHF, $\mathrm{H}_{\text {acfs }}$, that is $(m, 1,2)$-programmable and has a hash key consisting of $O(m \sqrt{\ell})$ group elements. By plugging $\mathrm{H}_{\text {acfs }}$ into our generic construction we immediately obtain standard-model signatures that achieve the same efficiency as the scheme of Yamada et al. [39]. Namely, they are the shortest standard model signature schemes with a public key of length $O(m \sqrt{\ell})$, that concretely allows for signatures of $302 \mathrm{bits}$ and a public key of $50 \mathrm{~KB}$. One of our two schemes recover the one in [39]. In this sense we provide a different conceptual approach to construct such signatures. While Yamada et al. obtained this result by going through an IBKEM, our solution revisits the original Hofheinz-Kiltz's idea of applying programmable functions.

We provide a detailed comparison of the schemes in Table 1.

\subsection{Other related work}

Hanaoka et al. [28] show that there cannot be any black-box construction of a (poly, 1)-PHF. The latter result has been overcome by the recent work of Freire et al. [23] who propose a

${ }^{3}$ [30] gives also a (1, poly)-programmable PHF which allows for different applications. 


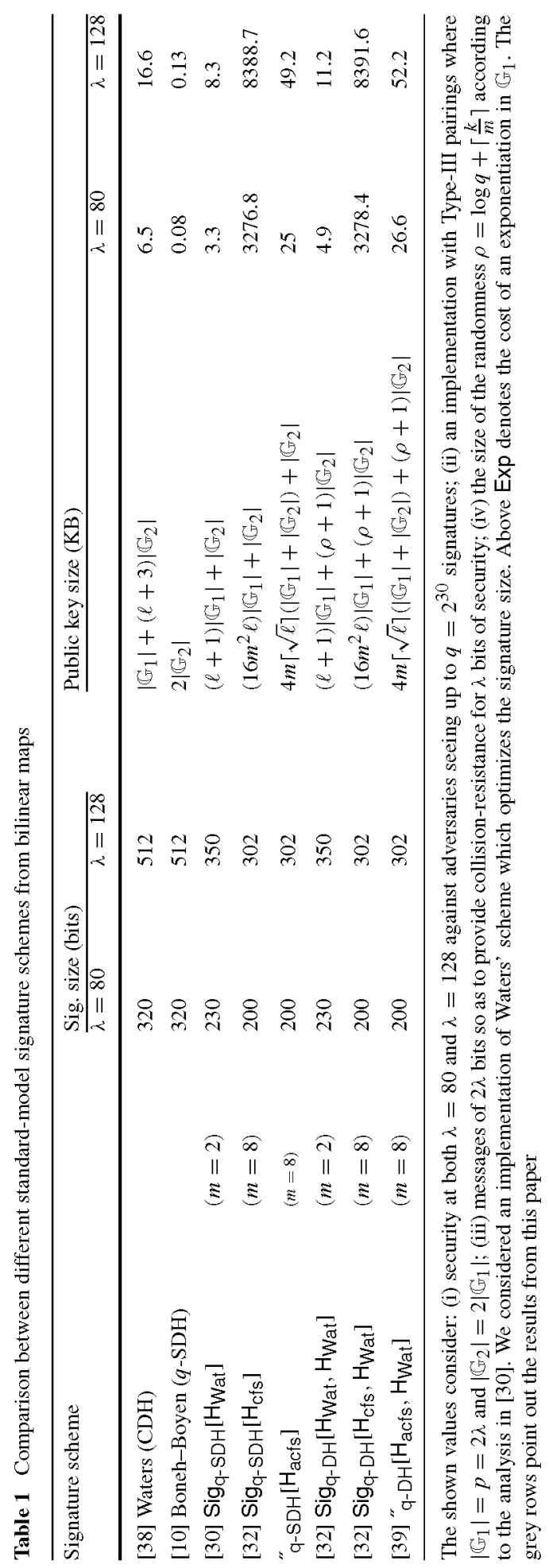


(poly, 1)-PHF based on multilinear maps slightly changing the definition of PHFs in order to work in the multilinear group setting. Their (poly, 1)-PHF leads to several applications, notably standard-model versions (over multilinear groups) of BLS signatures, the BonehFranklin IBE, and identity-based non-interactive key-exchange. While the notion of PHFs in the multilinear setting of [23] is different from our APHFs (with the main difference being that ours are secretly computable), it is worth noting that the two notions have some relation. As we discuss in Sect. 3.1, our APHFs indeed imply PHFs in the bilinear setting (though carrying the same degree of programmability).

The idea of using bilinear maps to reduce the size of public keys was used previously by Haralambiev et al. [29] in the context of public-key encryption, and by Yamada et al. [39] in the context of digital signatures. We note that our solutions use a similar approach in the construction of APHFs, which however also include the important novelty of programmable pseudorandomness, that turned out to be crucial in our proofs for the linearly-homomorphic signature.

With respect to programmable hash functions, it is also worth to mention the recent work of Zhang et al. [41] which proposes PHFs based on lattices and uses them to build short signatures and IBEs with short key size.

Our work is also related to the research line on linearly-homomorphic structure preserving signatures (LHSPS). Structure preserving signatures (SPSs) are a particular kind of cryptographic signatures in which messages, public key elements and signatures are all elements of a group over which there exists an efficiently computable bilinear map. In [35], Libert et al. introduced structure preserving signatures with linearly homomorphic properties: a bit more in detail, these signature schemes act exactly as other linearly homomorphic signatures, with the additional restriction that, as in SPSs, signatures and messages are vectors of group elements and the linearly homomorphic property holds with respect to the group operation. Moreover, the model adopted by works on LHSPSs is slightly different from the one of linearly-homomorphic signatures (as defined in $[11,22]$ and used in this paper). In LHSPSs one signs vectors along with a vector identifier, and security is defined so that an output by an adversary is considered a forgery if it consists of a valid signature on a vector which does not belong to the linear span of originally signed vectors. It is known that a LHSPS (following this model) could be used to construct a scheme that signs messages (which can also be vectors) in a dataset, as in the model considered by this paper: given a vector $\mathbf{v}_{i}$ at position $i$, one uses the LHSPS to sign the vector of group elements $g^{\mathbf{u}_{i}}$ where $\mathbf{u}_{i}=\mathbf{e}_{i} \| \mathbf{v}_{i}$, with $\mathbf{e}_{i}$ the $i$-th column of the identity matrix. However, since in all existing LHSPSs (in the standard model) the size of the public key is linear in the dimension of the signed vectors, using LHSPSs yields solutions with a public key linear in the dataset size. On the other hand, it remains an open problem to design a LHSPS whose public key is sub-linear in the dimension of the signed vectors. Indeed, our techniques for reducing the public key size do not seem to work in the structure-preserving setting due to the fact that messages are group elements whose discrete logarithms are not known.

\subsection{Publication note and organization}

This article is based on an earlier one [19] which appears in the proceedings of CRYPTO 2015. Besides including proofs and details that were missing from [19], in this version we added new results related to the context-hiding security of the proposed linearly-homomorphic signature scheme. More precisely, in this version we add a definition of context-hiding security, we propose a slightly modified version of our linearly-homomorphic scheme in [19], and we show that this scheme is also context-hiding secure. 
The paper is organized as follows. Section 2 provides the preliminary notions which are necessary in order to understand our work, such as bilinear groups and the complexity assumptions that we use to prove the security of our schemes. Section 3 is about the new concept of APHFs: we give definitions and propose two constructions of APHFs. Section 4 contains our results on linearly-homomorphic signatures: we recall their definition and then propose our construction and prove its security (including context hiding). Section 5 includes our results on short standard-model signatures. Finally, we defer the reader to the "Appendix" for the standard definition of digital signatures and an analysis of the proposed constant-size FDHI assumption.

\section{Preliminaries}

In this section, we review the notation and some basic definitions that we use in our work.

Notation We denote with $\lambda \in \mathbb{N}$ a security parameter. We say that a function $\epsilon$ is negligible if it vanishes faster than the inverse of any polynomial. If $S$ is a set, $x \stackrel{\$}{\leftarrow} S$ denotes the process of selecting $x$ uniformly at random in $S$. If $\mathcal{A}$ is a probabilistic algorithm, $x \stackrel{\$}{\leftarrow} \mathcal{A}(\cdot)$ denotes the process of running $\mathcal{A}$ on some appropriate input and assigning its output to $x$. Moreover, for a positive integer $n$, we denote by $[n]$ the set $\{1, \ldots, n\}$. Additionally, sometimes we will use a compact notation $g^{\mathbf{a}}$, for a group element $g \in \mathbb{G}$ and a vector $\mathbf{a}=\left(a_{1}, \ldots, a_{t}\right) \in \mathbb{Z}_{p}^{t}$, meaning the vector of group elements $\left(g^{a_{1}}, \ldots, g^{a_{t}}\right)$.

\subsection{Bilinear groups and complexity assumptions}

Let $\lambda \in \mathbb{N}$ be a security parameter and let $\mathcal{G}\left(1^{\lambda}\right)$ be an algorithm which takes as input the security parameter and outputs the description of (asymmetric) bilinear groups bgp $=$ $\left(p, \mathbb{G}_{1}, \mathbb{G}_{2}, \mathbb{G}_{T}, e, g_{1}, g_{2}\right.$ ) where $\mathbb{G}_{1}, \mathbb{G}_{2}$ and $\mathbb{G}_{T}$ are groups of the same prime order $p>2^{\lambda}$, $g_{1} \in \mathbb{G}_{1}$ and $g_{2} \in \mathbb{G}_{2}$ are two generators, and $e: \mathbb{G}_{1} \times \mathbb{G}_{2} \rightarrow \mathbb{G}_{T}$ is an efficiently computable, non-degenerate, bilinear map, and there is no efficiently computable isomorphism between $\mathbb{G}_{1}$ and $\mathbb{G}_{2}$. We call such an algorithm $\mathcal{G}$ a bilinear group generator. In the case $\mathbb{G}_{1}=\mathbb{G}_{2}$, the groups are said symmetric, else they are said asymmetric.

In our work we rely on specific computational and decisional assumptions in such bilinear groups.

Definition 1 ( $q$-Strong Diffie-Hellman [10]) Let $\mathcal{G}$ be a generator of asymmetric bilinear groups, let bgp $=\left(p, \mathbb{G}_{1}, \mathbb{G}_{2}, \mathbb{G}_{T}, g_{1}, g_{2}, e\right) \stackrel{\$}{\leftarrow} \mathcal{G}\left(1^{\lambda}\right)$ where $g_{1}, g_{2}$ are two random generators, and let $q=$ poly $(\lambda)$. We say that the $q$-Strong Diffie-Hellman assumption ( $q$-SDH) is $\epsilon$-hard for $\mathcal{G}$ if, for every PPT adversary $\mathcal{A}$,

$$
\operatorname{Adv}_{\mathcal{A}}^{q-S D H}(\lambda)=\operatorname{Pr}\left[\mathcal{A}\left(g_{1}, g_{1}^{z}, \ldots, g_{1}^{z^{q}}, g_{2}, g_{2}^{z}\right)=\left(c, g_{1}^{1 /(z+c)}\right) \mid z \stackrel{\$}{\leftarrow} \mathbb{Z}_{p}\right] \leq \epsilon
$$

Definition 2 ( $q$-Diffie-Hellman inversion $[9,36])$ Let $\mathcal{G}$ be a generator of asymmetric bilinear groups, let bgp $=\left(p, \mathbb{G}_{1}, \mathbb{G}_{2}, \mathbb{G}_{T}, g_{1}, g_{2}, e\right) \stackrel{\$}{\leftarrow} \mathcal{G}\left(1^{\lambda}\right)$ where $g_{1}, g_{2}$ are two random generators, and let $q=$ poly $(\lambda)$. We say that the $q$-Diffie-Hellman inversion assumption $(q$-DHI) is $\epsilon$-hard for $\mathcal{G}$ if, for every PPT adversary $\mathcal{A}$,

$$
\operatorname{Adv}_{\mathcal{A}}^{q-D H I}(\lambda)=\operatorname{Pr}\left[\mathcal{A}\left(g_{1}, g_{1}^{z}, g_{2}^{z}, \ldots, g_{1}^{z^{q}}, g_{2}^{z^{q}}\right)=g_{1}^{1 / z} \mid z \stackrel{\$}{\leftarrow} \mathbb{Z}_{p}\right] \leq \epsilon
$$

It is not hard to see that the above problem is equivalent to the one in which the adversary is given the same input and is challenged to compute the "next power" $g_{1}^{z^{q+1}}$. 
A weaker variant of the $q$-DHI assumption that we use in some of our proofs is the one in which the adversary receives only $g_{2}, g_{2}^{z}$ in the group $\mathbb{G}_{2}$. For coherence with [32] we call this assumption $q$-Diffie-Hellman ( $q-\mathrm{DH})$.

Definition 3 (External decisional Diffie-Hellman in $\mathbb{G}_{1}$ ) Let $\mathcal{G}$ be a generator of asymmetric bilinear groups, and let bgp $=\left(p, \mathbb{G}_{1}, \mathbb{G}_{2}, \mathbb{G}_{T}, g_{1}, g_{2}, e\right) \stackrel{\$}{\leftarrow} \mathcal{G}\left(1^{\lambda}\right)$. We say that the External Decisional Diffie-Hellman Assumption (XDDH) is $\epsilon$-hard in $\mathbb{G}_{1}$ if, for every PPT adversary $\mathcal{A}$, it holds

$$
\left|\operatorname{Pr}\left[\mathcal{A}\left(g_{1}, g_{1}^{a}, g_{1}^{b}, g_{1}^{a b}\right)=1 \mid a, b \stackrel{\$}{\leftarrow} \mathbb{Z}_{p}\right]-\operatorname{Pr}\left[\mathcal{A}\left(g_{1}, g_{1}^{a}, g_{1}^{b}, g_{1}^{c}\right)=1 \mid a, b, c \stackrel{\$}{\leftarrow} \mathbb{Z}_{p}\right]\right| \leq \epsilon
$$

Finally, we introduce the following static assumption over asymmetric bilinear groups, that we call "Flexible Diffie-Hellman Inversion" (FDHI) for its similarity to Flexible DiffieHellman [27]. As we discuss in "Appendix B", FDHI is hard in the generic bilinear group model.

Definition 4 (Flexible Diffie-Hellman Inversion Assumption) Let $\mathcal{G}$ be a generator of asymmetric bilinear groups, and let bgp $=\left(p, \mathbb{G}_{1}, \mathbb{G}_{2}, \mathbb{G}_{T}, g_{1}, g_{2}, e\right) \stackrel{\$}{\leftarrow} \mathcal{G}\left(1^{\lambda}\right)$. We say that the Flexible Diffie-Hellman Inversion (FDHI) Assumption is $\epsilon$-hard for $\mathcal{G}$ if for every PPT adversary $\mathcal{A}$ :

$$
\begin{aligned}
\operatorname{Adv}_{\mathcal{A}}^{F D H I}(\lambda) & =\operatorname{Pr}\left[W \in \mathbb{G}_{1} \backslash\left\{1_{\mathbb{G}_{1}}\right\} \wedge W^{\prime}\right. \\
& \left.=W^{\frac{1}{z}}:\left(W, W^{\prime}\right) \leftarrow \mathcal{A}\left(g_{1}, g_{2}, g_{2}^{z}, g_{2}^{v}, g_{1}^{\frac{z}{v}}, g_{1}^{r}, g_{1}^{\frac{r}{v}}\right) \mid z, r, v \stackrel{\$}{\leftarrow} \mathbb{Z}_{p}\right] \leq \epsilon .
\end{aligned}
$$

\section{Asymmetric programmable hash functions}

In this section we present our new notion of asymmetric programmable hash functions.

Let bgp $=\left(p, \mathbb{G}_{1}, \mathbb{G}_{2}, \mathbb{G}_{T}, g_{1}, g_{2}, e\right)$ be a family of asymmetric bilinear groups induced by a bilinear group generator $\mathcal{G}\left(1^{\lambda}\right)$ for a security parameter $\lambda \in \mathbb{N}^{4}$ An asymmetric group hash function $\mathrm{H}: \mathcal{X} \rightarrow \mathbb{G}_{1}$ consists of three PPT algorithms (H.Gen, H.PriEval, H.PubEval) working as follows:

H.Gen $\left(1^{\lambda}\right.$, bgp $) \rightarrow$ (sek, pek): on input the security parameter $\lambda \in \mathbb{N}$ and a bilinear group description bgp, the PPT key generation algorithm outputs a (secret) evaluation key sek and a (public) evaluation key pek.

H.PriEval(sek, $X) \rightarrow Y \in \mathbb{G}_{1}$ : given the secret evaluation key sek and an input $X \in \mathcal{X}$, the deterministic evaluation algorithm returns an output $Y=\mathrm{H}(X) \in \mathbb{G}_{1}$.

H.PubEval(pek, $X) \rightarrow \hat{Y} \in \mathbb{G}_{T}$ : on input the public evaluation key pek and an input $X \in$ $\mathcal{X}$, the public evaluation algorithm outputs a value $\hat{Y} \in$ $\mathbb{G}_{T}$ such that $\hat{Y}=e\left(\mathrm{H}(X), g_{2}\right)$.

For asymmetric hash functions satisfying the syntax described above, we define two different properties that model their possible programmability.

The first property is a generalization of the notion of programmable hash functions of $[30,31]$ to our asymmetric setting (i.e., where the function is only secretly-computatble), and

4 Our definition can be easily adapted to work in symmetric bilinear groups where $\mathbb{G}_{1}=\mathbb{G}_{2}$. 
to the more specific setting of bilinear groups. The basic idea is that it is possible to generate the function in a trapdoor-mode that allows one to express every output of $H$ in relation to some specified group elements. In particular, the most useful fact of programmability is that for two arbitrary disjoint sets of inputs $\bar{X}, \bar{Z} \subset \mathcal{X}$, the joint probability that some of these group elements appear in $H(Z), \forall Z \in \bar{Z}$ and do not appear in $H(X), \forall X \in \bar{X}$ is significant.

Definition 5 (Asymmetric programmable hash functions) An asymmetric group hash function $\mathrm{H}=(\mathrm{H}$. Gen, H.PriEval, H.PubEval) is $(m, n, d, \gamma, \delta)$-programmable if there exist an efficient trapdoor generation algorithm $\mathrm{H}$. TrapGen and an efficient trapdoor evaluation algorithm $\mathrm{H}$. TrapEval such that:

Syntax:

Correctness:

Statistically-close trapdoor keys:

Well distributed logarithms:

\begin{abstract}
H.TrapGen $\left(1^{\lambda}\right.$, bgp, $\left.\hat{g}_{1}, \hat{h}_{1}, \hat{g}_{2}, \hat{h}_{2}\right) \rightarrow(\mathrm{td}$, pek $)$ takes as input the security parameter $\lambda$, bilinear group description bgp and group elements $\hat{g}_{1}, \hat{h}_{1} \in$ $\mathbb{G}_{1}, \hat{g}_{2}, \hat{h}_{2} \in \mathbb{G}_{2}$, and it generates a public hash key pek along with a trapdoor td. H.TrapEval (td, $X) \rightarrow$ $\mathbf{c}_{X}$ takes as input the trapdoor information $t d$ and an input $X \in \mathcal{X}$, and outputs a vector of integer coefficients $\mathbf{c}_{X}=\left(c_{0}, \ldots, c_{d^{\prime}}\right) \in \mathbb{Z}^{d^{\prime}}$ of a 2-variate polynomial $c_{X}\left(y_{1}, y_{2}\right)$ of degree $\leq d$.

For all group elements $\hat{g}_{1}, \hat{h}_{1} \in \mathbb{G}_{1}, \hat{g}_{2}, \hat{h}_{2} \in \mathbb{G}_{2}$ such that $\hat{h}_{1}=\hat{g}_{1}^{y_{1}}$ and $\hat{h}_{2}=\hat{g}_{2}^{y_{2}}$ for some $y_{1}, y_{2} \in \mathbb{Z}_{p}$, for all trapdoor keys (td, pek) H.TrapGen $\left(1^{\lambda}, \hat{g}_{1}, \hat{h}_{1}, \hat{g}_{2}, \hat{h}_{2}\right)$, and for all inputs $X \in$ $\mathcal{X}$, if $\mathbf{c}_{X} \leftarrow \mathrm{H}$. TrapEval (td, $\left.X\right)$, then

$$
H(X)=\hat{g}_{1}^{c_{X}\left(y_{1}, y_{2}\right)}
$$
\end{abstract}

For all generators $\hat{g}_{1}, \hat{h}_{1} \in \mathbb{G}_{1}, \hat{g}_{2}, \hat{h}_{2} \in \mathbb{G}_{2}$ and for all (sek, pek) $\stackrel{\$}{\leftarrow}$ H.Gen $\left(1^{\lambda}\right)$, (td, pek') $\stackrel{\$}{\leftarrow}$ H.TrapGen $\left(1^{\lambda}, \hat{g}_{1}, \hat{h}_{1}, \hat{g}_{2}, \hat{h}_{2}\right)$, the distribution of the public keys pek and pek' is within statistical distance $\gamma$. For all $\hat{g}_{1}, \hat{h}_{1} \in \mathbb{G}_{1}, \hat{g}_{2}, \hat{h}_{2} \in \mathbb{G}_{2}$, all keys (td, pek) $\stackrel{\$}{\leftarrow}$ H.TrapGen $\left(1^{\lambda}, \hat{g}_{1}, \hat{h}_{1}, \hat{g}_{2}, \hat{h}_{2}\right)$, and all inputs $X_{1}, \ldots, X_{m} \in \mathcal{X}$ and $Z_{1}, \ldots, Z_{n} \in \mathcal{X}$ such that $X_{i} \neq Z_{j}$ for all $i, j$, we have

$$
\begin{aligned}
\operatorname{Pr}\left[c_{X_{1}, 0}\right. & =\cdots=c_{X_{m}, 0} \\
& \left.=0 \wedge c_{Z_{1}, 0}, \ldots, c_{Z_{n}, 0} \neq 0\right] \geq \delta
\end{aligned}
$$

where $\quad \mathbf{c}_{X_{i}} \leftarrow \mathrm{H}$. TrapEval $\left(\mathrm{td}, X_{i}\right) \quad$ and $\quad \mathbf{c}_{Z_{j}} \leftarrow \mathrm{H}$. TrapEval(td, $Z_{j}$ ), and $c_{X_{i}, 0}$ (resp. $c_{Z_{j}, 0}$ ) is the coefficient of the term of degree 0 . The probability is over the trapdoor $t d$ that was produced along with pek.

If $\gamma$ is negligible and $\delta$ is noticeable we simply say that $\mathrm{H}$ is $(m, n, d)$-programmable. Furthermore, if $m$ (resp. $n$ ) is an arbitrary polynomial in $\lambda$, then we say that $\mathrm{H}$ is (poly, $n, d$ )programmable (resp. ( $m$, poly, $d$ )-programmable). Finally, if $\mathrm{H}$ admits trapdoor algorithms that satisfy only the first three properties, then $\mathrm{H}$ is said simply $(d, \gamma)$-programmable. Note that any $\mathrm{H}$ that is $(m, n, d, \gamma, \delta)$-programmable is also $(d, \gamma)$-programmable. 
Programmable pseudorandomness The second main programmability property that we define for asymmetric hash functions is quite different from the previous one. It is called programmable pseudorandomness, and very intuitively it says that, when using the hash function in trapdoor mode, it is possible to "embed" a PRF into it. More precisely, the trapdoor algorithms satisfy programmable pseudorandomness if they allow to generate keys such that even by observing pek and $H(X)$ for a bunch of inputs $X$, then the elements $g_{1}^{c X, 0}$ look random. The formal definition follows:

Definition 6 (Asymmetric hash functions with programmable pseudorandomness) An asymmetric hash function $\mathrm{H}=(\mathrm{H}$. Gen, H.PriEval, H.PubEval) has $(d, \gamma, \epsilon)$-programmable pseudorandomness if there exist efficient trapdoor algorithms $\mathrm{H}$. TrapGen, $\mathrm{H}$. TrapEval that satisfy the properties of syntax, correctness, and $\gamma$-statistically-close trapdoor keys as in Definition 5 , and additionally satisfy the following property with parameter $\epsilon$ :

Pseudorandomness: Let $b \in\{0,1\}$ and let $\operatorname{Exp}_{\mathcal{A}, \mathrm{H}}^{P R-b}(\lambda)$ be the following experiment between an adversary $\mathcal{A}$ and a challenger.

1. Generate bgp $\stackrel{\$}{\leftarrow} \mathcal{G}\left(1^{\lambda}\right)$, and run $\mathcal{A}$ (bgp), that outputs two generators $h_{1} \in \mathbb{G}_{1}, h_{2} \in \mathbb{G}_{2}$.

2. Compute (td, pek) $\stackrel{s}{\leftarrow} \mathrm{H}$. TrapGen $\left(1^{\lambda}, g_{1}, h_{1}, g_{2}, h_{2}\right)$ and run $\mathcal{A}$ (pek) with access to the following oracle:

- If $b=0, \mathcal{A}$ is given $\mathcal{O}(\cdot)$ that on input $X \in \mathcal{X}$ returns $\mathrm{H}(X)=g_{1}^{c_{X}\left(y_{1}, y_{2}\right)}$ and $g_{1}^{c_{X, 0}}$, where $\mathbf{c}_{X} \leftarrow \mathrm{H}$. TrapEval (td, $\left.X\right)$;

- If $b=1, \mathcal{A}$ is given $\mathcal{R}(\cdot)$ that on input $X \in \mathcal{X}$ returns $\mathrm{H}(X)=g_{1}^{c_{X}\left(y_{1}, y_{2}\right)}$ and $g_{1}^{r_{X}}$, for a randomly chosen $r_{X} \stackrel{\$}{\leftarrow} \mathbb{Z}_{p}$ (which is unique for every $X \in \mathcal{X}$ ).

3. At the end the adversary outputs a bit $b^{\prime}$, and $b^{\prime}$ is returned as the output of the experiment. Then we say that $\mathrm{H}$. TrapGen, $\mathrm{H}$. TrapEval satisfy pseudorandomness for $\epsilon$, if for all PPT $\mathcal{A}$

$$
\left|\operatorname{Pr}\left[\operatorname{Exp}_{\mathcal{A}, H}^{P R H-0}(\lambda)=1\right]-\operatorname{Pr}\left[\operatorname{Exp}_{\mathcal{A}, H}^{P R H-1}(\lambda)=1\right]\right| \leq \epsilon
$$

where the probabilities are taken over all the random choices of TrapGen, the oracle $\mathcal{R}$ and the adversary $\mathcal{A}$.

Remark 1 (On the mutual existence of programmability and programmable pseudorandomness) We stress that the two properties of programmability and programmable pseudorandomness defined above are mutually exclusive. Precisely, an APHF can have a pair of trapdoor algorithms (TrapGen, TrapEval) that admits either $(m, n, d, \gamma, \delta)$-programmability (for non-negligible $\delta$ ), or $(d, \gamma, \epsilon)$-programmable pseudorandomness (for negligible $\epsilon$ ). Intuitively, the reason why the same trapdoor algorithms cannot satisfy both properties is that $(m, n, \delta, \gamma)$-programmability implies that for any elements $X_{1}, \ldots, X_{m} \in \mathcal{X}$ it holds $c_{X_{i, 0}}=0$ with non negligible probability $\delta$. However, if this holds then programmable pseudorandomness can be trivially broken, since $g_{1}^{C_{X}, 0}=1$ with non negligible probability $\delta$.

On the other hand, it is quite interesting to observe that the same function can enjoy both properties through different, appropriate, pairs of trapdoor algorithms. In fact, an asymmetric group hash function can have a pair of trapdoor algorithms (TrapGen, TrapEval) for which $(m, n, \delta, \gamma)$-programmability holds, and another pair of trapdoor algorithms (TrapGen', TrapEval') for which $(d, \gamma, \delta)$-programmable pseudorandomness holds. Then, since all trapdoor generations produce keys that are statistically indistinguishable from the real ones it follows that also the two trapdoor modes are statistically indistinguishable. In a 
nutshell, this means that the same function can be programmed in different modes in different steps of a security proof, a property which turns out to be very useful, for example, in our proofs of Sect. 4.4 .

Other variants of programmability Here we define two other variants of the programmability notion given in Definition 5 .

Weak programmability We consider a weak version of the above programmability property in which one fixes at key generation time the $n$ inputs $Z_{j}$ on which $c_{Z_{j}, 0} \neq 0$.

Definition 7 (Asymmetric weakly-programmable hash functions) An asymmetric group hash function $\mathrm{H}=(\mathrm{H}$. Gen, H.PriEval, H.PubEval) is weakly $(m, n, d, \gamma, \delta)$-programmable if there exist efficient trapdoor generation $H$. TrapGen and trapdoor evaluation $H$. TrapEval algorithms such that:

- Syntax: H.TrapGen( $1^{\lambda}$, bgp, $\left.\hat{g}_{1}, \hat{h}_{1}, \hat{g}_{2}, \hat{h}_{2}, Z_{1}, \ldots, Z_{n}\right) \rightarrow$ (td, pek) takes as input the security parameter $\lambda$, bilinear group description bgp, group elements $\hat{g}_{1}, \hat{h}_{1} \in$ $\mathbb{G}_{1}, \hat{g}_{2}, \hat{h}_{2} \in \mathbb{G}_{2}$, and a set of $n$ inputs $Z_{1}, \ldots, Z_{n} \in \mathcal{X}$. It generates a public hash key pek along with a trapdoor td. H.TrapEval(td, $X) \rightarrow \mathbf{c}_{X}$ works exactly as in Definition 5.

- The properties of correctness and statistically-close trapdoor keys hold as in Definition 5 . The property of well-distributed logarithms is also the same except that the inputs $Z_{1}, \ldots, Z_{n}$ are the ones fixed as input to $H$.TrapGen.

Degree-d programmability In our work we also consider a variant of the above definition in which the property of well distributed logarithms is stated with respect to the degree$d$ coefficients of the polynomials generated by $\mathrm{H}$. TrapEval. In this case, we say that $\mathrm{H}$ is $(m, n, d, \gamma, \delta)$-degree-d-programmable.

\subsection{Relation with existing notions}

Before describing our realizations of APHFs, we discuss here the relation between our new notion and two existing notions of programmable hash functions: the original one by Hofheinz and Kiltz [30], recalled in "Appendix C", and its adaptation to the multilinear setting recently proposed by Freire et al. [23].

When working over bilinear groups, the notion of programmable hash functions of [30] is essentially a special case of ours. The main differences are: (1) PHFs are publicly computable, (2) the trapdoor algorithms work with only two generators $\hat{g}, \hat{h}$ and every output of the function can be expressed as a linear function $\hat{g}^{a} \hat{h}^{b}$ of these two generators. As we formally state in the following theorem, a standard PHF is an APHF for $d=1$ :

Theorem 1 Let $\hat{\mathrm{H}}=$ (PHF.Gen, PHF.Eval) be an ( $m, n, \gamma, \delta)$-programmable hash function such that $\mathrm{H}: \mathcal{X} \rightarrow \mathbb{G}_{1}$. Define $\mathrm{H} . \mathrm{Gen}=$ PHF.Gen, H.PriEval = PHF.Eval and (informally) H.PubEval $=e\left(\right.$ PHF.Eval, $\left.g_{2}\right)$. Then $\mathrm{H}=(\mathrm{H}$. Gen, H.PriEval, H.PubEval) is an asymmetric $(m, n, 1, \gamma, \delta)$-programmable hash function.

The proof is straightforward and is omitted.

Second, we analyze the relation between asymmetric hash functions and the PHFs in the multilinear setting introduced in [23]. Informally, for a setting of leveled multilinear groups $\mathbb{G}_{1}, \ldots, \mathbb{G}_{\ell},[23]$ considers a group hash function $\hat{\mathrm{H}}: \mathcal{X} \rightarrow \mathbb{G}_{\ell}$. Then, $\hat{\mathrm{H}}$ is said $(m, n)$ programmable if there exist two trapdoor algorithms PHF.TrapGen, PHF. TrapEval such that: PHF.TrapGen $\left(1^{\lambda}, g_{1}, \ldots, g_{\ell}, h\right)$ takes as input $g_{i}, h \in \mathbb{G}_{1}$ with $h \neq 1$ and outputs a 
trapdoor td and hash key hk; PHF. TrapEval(td, $X)$ on input $X$ outputs an integer $a_{X}$ and an element $B_{X} \in \mathbb{G}_{\ell-1}$ such that $\mathrm{H}(X)=e\left(g_{1}, \ldots, g_{\ell}\right)^{a_{X}} e\left(B_{X}, h\right) \in \mathbb{G}_{\ell}$. If we consider leveled bilinear groups where $\mathbb{G}=\mathbb{G}_{1}$ and $\mathbb{G}_{T}=\mathbb{G}_{2}$, then asymmetric programmable hash functions (for $d \leq 2$ ) imply PHFs in the (symmetric) bilinear group setting:

Theorem 2 Let $\mathbb{G}, \mathbb{G}_{T}$ be symmetric bilinear groups, and let $\mathrm{H}=(\mathrm{H} . \mathrm{Gen}, \mathrm{H}$.PriEval, $\mathrm{H}$. PubEval) be an asymmetric ( $m, n, 2, \gamma, \delta$ )-programmable hash function such that $\mathrm{H}$ : $\mathcal{X} \rightarrow \mathbb{G}$. Define PHF.Gen $=$ H.Gen and PHF.Eval $=$ H.PubEval. Then $\hat{\mathrm{H}}=$ (PHF.Gen, PHF.Eval) is an $(m, n, \gamma, \delta)$-programmable hash function in the bilinear setting.

The proof is fairly easy. Here we provide a sketch. Basically, by assuming that $H$ is programmable, we have to show two algorithms PHF. TrapGen, PHF. TrapEval that satisfy the programmability of $\hat{H}$ in the bilinear setting:

PHF.TrapGen $\left(1^{\lambda}, g, h\right)$ : run (td, pek) $\stackrel{\$}{\leftarrow}$. TrapGen $\left(1^{\lambda}, g, h\right)$ and output (td, pek).

PHF. TrapEval(td, $X)$ : run $\mathbf{c}_{X} \leftarrow H$. TrapEval(td, $\left.X\right)$ to generate the coefficient of a degree-2 polynomial $c_{X}(y)$ where $y=D \log _{g}(h)$. Then output $a_{X}=c_{X, 0}$, and $B_{X}=g^{c_{X, 1}} h^{c_{X, 2}}$.

It is easy to see that if $\mathbf{c}_{X}$ is such that $H(X)=g^{c_{X, 0}+c_{X, 1} y+c_{X, 2} y^{2}}$ then

$$
\hat{\mathrm{H}}(X)=e(\mathrm{H}(X), g)=e(g, g)^{c_{X, 0}} e\left(g^{c_{X, 1}+c_{X, 2} y}, g^{y}\right)=e(g, g)^{a_{X}} e\left(B_{X}, h\right)
$$

Finally, the $(m, n, \gamma, \delta)$-programmability of $\hat{\mathrm{H}}$ is immediately implied by the well distribution of the discrete logarithms in $\mathrm{H}$ for parameters $(m, n, 2, \gamma, \delta)$.

\subsection{An asymmetric programmable hash function based on cover-free sets}

In this section we present the construction of an asymmetric hash function, $\mathrm{H}_{\text {acts }}$, based on cover-free sets. Our construction uses ideas similar to the ones used by Hofheinz, Jager and Kiltz [32] to design a (regular) programmable hash function. Our construction extends these ideas with a technique that allows us to obtain a much shorter public key. Concretely, for binary inputs of size $\ell$, the programmable hash function $\mathrm{H}_{\mathrm{cfs}}$ in [32] is $(m, 1)$-programmable with a hash key of length $O\left(\ell m^{2}\right)$. In contrast, our new construction $\mathrm{H}_{\text {acfs }}$ is $(m, 1)$-programmable with a hash key of length $O(m \sqrt{\ell})$. While such improvement is obtained the price of obtaining the function in the secret-key model, our results of Sect. 5 show that asymmetric programmable hash are still useful to build short bilinear-map signatures, whose efficiency, in terms of signature's and key's length matches that of state-of-the-art schemes [39].

Before proceeding with describing our function, below we recall the notion of cover-free sets.

Cover-free families If $S, V$ are sets, we say that $S$ does not cover $V$ if $S \nsupseteq V$. Let $T, m, s$ be positive integers, and let $F=\left\{F_{i}\right\}_{i \in[s]}$ be a family of subsets of [T]. A family $F$ is said to be $m$-cover-free over [T], if for any subset $I \subseteq[s]$ of cardinality at most $m$, then the union $\cup_{i \in I} F_{i}$ does not cover $F_{j}$ for all $j \notin I$. More formally, for any $I \subseteq[s]$ such that $|I| \leq m$, and any $j \notin I, \cup_{i \in I} F_{i} \nsupseteq F_{j}$. Furthermore, we say that $F$ is $w$-uniform if every subset $F_{i}$ in the family have size $w$. In our construction, we use the following fact from [21,34]:

Lemma $1[21,34]$ There is a deterministic polynomial time algorithm that, on input integers $s=2^{\ell}$ and $m$, returns $w, T, F$ where $F=\left\{F_{i}\right\}_{i \in[s]}$ is a $w$-uniform, $m$-cover-free family over $[T]$, for $w=T / 4 m$ and $T \leq 16 m^{2} \ell$. 
The construction of $\mathrm{H}_{\text {acts }}$ Let $\mathcal{G}\left(1^{\lambda}\right)$ be a bilinear group generator, let bgp $=\left(p, \mathbb{G}_{1}, \mathbb{G}_{2}\right.$, $\left.\mathbb{G}_{T}, g_{1}, g_{2}, e\right)$ be an instance of bilinear group parameters generated by $\mathcal{G}$. Let $\ell=\ell(\lambda)$ and $m=m(\lambda)$ be two polynomials in the security parameter. We set $s=2^{\ell}, T=16 m^{2} \ell$, and $w=T / 4 m$ as for Lemma 1 , and define $t=\lceil\sqrt{T}\rceil$. Note that every integer $k \in[T]$ can be written as a pair of integers $(i, j) \in[t] \times[t]$ using some canonical mapping. For the sake of simplicity, sometimes we abuse notation and write $(i, j) \in[T]$ where $i, j \in[t]$.

In the following we describe the asymmetric hash function $\mathrm{H}_{\text {acfs }}=(H$. Gen, H.PriEval, H.PubEval) that maps $\mathrm{H}_{\text {acts }}: \mathcal{X} \rightarrow \mathbb{G}_{1}$ where $\mathcal{X}=\{0,1\}^{\ell}$. In particular, every input $X \in\{0,1\}^{\ell}$ is associated to a set $F_{i}, i \in\left[2^{\ell}\right]$, by interpreting $X$ as an integer in $\left\{0, \ldots, 2^{\ell}-1\right\}$ and by setting $i=X+1$. We call $F_{X}$ such subset associated to $X$.

H.Gen $\left(1^{\lambda}, \operatorname{bgp}\right)$ :

H.PriEval(sek, $X)$ :

H.PubEval(pek, $X)$ : for $i=1$ to $t$, sample $\alpha_{i}, \beta_{i} \stackrel{\$}{\leftarrow} \mathbb{Z}_{p}$ and compute $A_{i}=$ $g_{1}^{\alpha_{i}}, B_{i}=g_{2}^{\beta_{i}}$. Finally, set sek $=\left\{\alpha_{i}, \beta_{i}\right\}_{i=1}^{t}$, pek $=$ $\left\{A_{i}, B_{i}\right\}_{i=1}^{t}$, and return (sek, pek).

first, compute the subset $F_{X} \subseteq[T]$ associated to $X \in\{0,1\}^{\ell}$, and then return

$$
Y=g_{1}^{\sum_{(i, j) \in F_{X}} \alpha_{i} \beta_{j}} \in \mathbb{G}_{1}
$$

let $F_{X} \subseteq[T]$ be the subset associated to $X$, and compute

$$
\hat{Y}=\prod_{(i, j) \in F_{X}} e\left(A_{i}, B_{j}\right)=e\left(\mathrm{H}(X), g_{2}\right)
$$

Theorem 3 Let $\mathcal{G}$ be a bilinear group generator. The hash function $\mathrm{H}_{\text {acts }}$ described above is an asymmetric $(m, n, d, \gamma, \delta)$-programmable hash function with $n=1, d=2, \gamma=0$ and $\delta=1 / T$.

Proof First, we describe the trapdoor algorithms:

H.TrapGen $\left(1^{\lambda}\right.$, bgp, $\left.\hat{g}_{1}, \hat{h}_{1}, \hat{g}_{2}, \hat{h}_{2}\right): \quad$ first, sample $a_{i}, b_{i} \stackrel{\$}{\leftarrow} \mathbb{Z}_{p}$ for all $i \in[t]$, and pick a random index $\tau \stackrel{\$}{\leftarrow}[T]$. Parse $\tau=\left(i^{*}, j^{*}\right) \in$ $[t] \times[t]$. Next, set $A_{i^{*}}=\hat{g}_{1} \hat{h}_{1}^{a_{i}{ }^{*}}, B_{j^{*}}=\hat{g}_{2} \hat{h}_{2}^{b_{j^{*}}}$, $A_{i}=\hat{h}_{1}^{a_{i}}, \forall i \neq i^{*}$, and $B_{j}=\hat{h}_{2}^{b_{j}}, \forall j \neq j^{*}$. Finally, set $\mathrm{td}=\left(\tau,\left\{a_{i}, b_{i}\right\}_{i=1}^{t}\right)$, pek $=\left\{A_{i}, B_{i}\right\}_{i=1}^{t}$, and output (td, pek).

H. TrapEval(td, $X)$ :

first, compute the subset $F_{X} \subseteq[T]$ associated to $X \in\{0,1\}^{\ell}$, and then return the coefficients of the degree-2 polynomial

$$
c_{X}\left(y_{1}, y_{2}\right)=\sum_{(i, j) \in F_{X}} \alpha_{i}\left(y_{1}\right) \cdot \beta_{j}\left(y_{2}\right)
$$

where every $\alpha_{i}\left(y_{1}\right)$ (resp. $\left.\beta_{j}\left(y_{2}\right)\right)$ is the discrete logarithm of $A_{i}$ (resp. $B_{j}$ ) in base $\hat{g}_{1}$ (resp. $\hat{g}_{2}$ ), viewed as a degree- 1 polynomial in the unknown $y_{1}$ (resp. $\left.y_{2}\right)$.

Now, we show that the two trapdoor algorithms described above satisfy the four properties of Definition 5. First, syntax and correctness immediately follow by construction. Second, observe that each element $A_{i}$ (resp. $B_{j}$ ) in pek is a uniformly distributed group element in 
$\mathbb{G}_{1}$ (resp. $\mathbb{G}_{2}$ ), exactly as in the output of H.Gen, hence $\gamma=0$. Third, we show that the algorithms allow for well distributed logarithms for the case $n=1$. Let $X_{1}, \ldots, X_{m}, Z \in \mathcal{X}$ such that $Z \neq X_{i}$ for all $i$. From the $m$-cover-free property of $F$ we have that there exist an index $\tau^{\prime} \in F_{Z}$ such that $\tau^{\prime} \notin \cup_{i=1}^{m} F_{X_{i}}$. Since $\tau$ is chosen uniformly at random in $[T]$, we have that $\tau=\tau^{\prime}$ with probability $\delta=1 / T$. Now, assume that $\tau^{\prime}=\tau=\left(i^{*}, j^{*}\right) \in[t] \times[t]$. Then for all $(i, j) \neq\left(i^{*}, j^{*}\right)$ it holds that the degree-0 coefficient of $c\left(y_{1}, y_{2}\right)=\alpha_{i}\left(y_{1}\right) \beta_{j}\left(y_{2}\right)$ is $c_{0}=0$, whereas for $\left(i^{*}, j^{*}\right)$ the degree-0 coefficient of $c^{*}\left(y_{1}, y_{2}\right)=\alpha_{i}\left(y_{1}\right) \beta_{j^{*}}\left(y_{2}\right)=$ $\left(a_{i} y_{1}+1\right)\left(b_{j^{*}} y_{2}+1\right)$, is $c_{0}^{*}=1$. Therefore, we have that $c_{X_{i}, 0}=0, \forall i \in[m]$ and $c_{Z, 0}=1$ holds with probability $\delta$.

\subsection{An asymmetric programmable hash function with small domain}

In this section, we present the construction of an asymmetric hash function, $\mathrm{H}_{\text {sqrt }}$, whose domain is of polynomial size $T$. $\mathrm{H}_{\text {sqrt }}$ has a public key of length $O(\sqrt{T})$, and it turns out to be very important for obtaining our linearly-homomorphic signature scheme with short public key presented in Sect. 4. Somewhat interestingly, we show that this new function $\mathrm{H}_{\text {sqrt }}$ satisfies several programmability properties, that make it useful in the context of various security proofs.

Let $\mathcal{G}\left(1^{\lambda}\right)$ be a bilinear group generator, let $T=$ poly $(\lambda)$ and $t=\lceil\sqrt{T}\rceil$. The hash function $\mathrm{H}_{\text {sqrt }}=\left(\mathrm{H}\right.$. Gen, H.PriEval, H.PubEval) that maps $\mathrm{H}_{\text {sqrt }}: \mathcal{X} \rightarrow \mathbb{G}_{1}$ with $\mathcal{X}=[T]$ is defined as follows.

H.Gen $\left(1^{\lambda}\right.$, bgp $): \quad$ for $i=1$ to $t$, sample $\alpha_{i}, \beta_{i} \stackrel{\$}{\leftarrow} \mathbb{Z}_{p}$ and compute $A_{i}=g_{1}^{\alpha_{i}}, B_{i}=$ $g_{2}^{\beta_{i}}$. Finally, set sek $=\left\{\alpha_{i}, \beta_{i}\right\}_{i=1}^{t}$, pek $=\left\{A_{i}, B_{i}\right\}_{i=1}^{t}$, and return(sek, pek).

H.PriEval(sek, $X)$ : $\quad$ first, write $X \in[T]$ as a pair of integer $(i, j) \in[t] \times[t]$, and then return

$$
Y=g_{1}^{\alpha_{i} \beta_{j}} \in \mathbb{G}_{1}
$$

H.PubEval(pek, $X)$ : $\quad$ let $X=(i, j)$. The public evaluation algorithm returns

$$
\hat{Y}=e\left(A_{i}, B_{j}\right)=e\left(\mathrm{H}(X), g_{2}\right)
$$

Programmable pseudorandomness of $\mathrm{H}_{\text {sqrt }}$ Here we show that $\mathrm{H}_{\text {sqrt }}$ satisfies the programmable pseudorandomness property of Definition 6.

Theorem 4 Let $\mathbb{G}_{1}$ be a bilinear group of order $p$ over which the XDDH assumption is $\epsilon^{\prime}$-hard. Then the asymmetric hash function $\mathrm{H}_{\mathrm{sqr}}$ described above satisfies $(2,0, \epsilon)$ programmable pseudorandomness with $\epsilon=T \cdot \epsilon^{\prime}$. Furthermore, in the case when $h_{1}=$ $1 \in \mathbb{G}_{1}$ or $h_{1}=g_{1}, \mathrm{H}_{\text {sqrt }}$ has $(1,0, \epsilon)$-programmable pseudorandomness.

Proof First, we describe the trapdoor algorithms:

H. TrapGen $\left(1^{\lambda}, g_{1}, h_{1}, g_{2}, h_{2}\right): \quad$ first, sample $a_{i}, r_{i}, s_{i}, b_{i} \stackrel{\$}{\leftarrow} \mathbb{Z}_{p}$ for all $i \in[t]$ and then set $A_{i}=h_{1}^{r_{i}} g_{1}^{a_{i}}, B_{i}=h_{2}^{s_{i}} g_{2}^{b_{i}}$. Finally, set td $=$ $\left(\left\{a_{i}, r_{i}, s_{i}, b_{i}\right\}_{i=1}^{t}\right)$, pek $=\left\{A_{i}, B_{i}\right\}_{i=1}^{t}$, and output (td, pek).

H. TrapEval(td, $X)$ :

let $X=(i, j)$, and then return the coefficients of the degree-2 polynomial

$$
c_{X}\left(y_{1}, y_{2}\right)=\left(y_{1} r_{i}+a_{i}\right)\left(y_{2} s_{j}+b_{j}\right)
$$


First, it is easy to see that the two algorithms satisfy the syntax and correctness properties. Also, in the case $h_{1}=1$ (i.e., $y_{1}=0$ ) or $h_{1}=g_{1}$ (i.e., $y_{1}=1$ ), we obtain a degree- 1 polynomial $c_{X}\left(y_{2}\right)$. Second, observe that each element $A_{i}$ (resp. $B_{i}$ ) in pek is a uniformly distributed group element in $\mathbb{G}_{1}$ (resp. $\mathbb{G}_{2}$ ), as in H.Gen, hence $\gamma=0$. Third, we show that the function satisfies the pseudorandomness property under the assumption that XDDH holds in $\mathbb{G}_{1}$. The main observation is that for every $X=(i, j)$, we have $c_{X, 0}=a_{i} b_{j}$ where all the values $b_{i}$ are uniformly distributed and information-theoretically hidden to an adversary who only sees pek. In particular, this holds even if $h_{1}=1$.

To prove the pseudorandomness we make use of Lemma 2 below, which shows that for a uniformly random choice of $\mathbf{a}, \mathbf{b} \stackrel{\$}{\leftarrow} \mathbb{Z}_{p}^{t}, c \stackrel{\$}{\leftarrow} \mathbb{Z}_{p}^{t \times t}$ the distributions $\left(g_{1}^{\mathbf{a}}, g_{1}^{\mathbf{a} \cdot \mathbf{b}^{\top}}\right) \in \mathbb{G}_{1}^{t \times(t+\mathbf{1})}$ and $\left(g_{1}^{\mathbf{a}}, g_{1}^{c}\right) \in \mathbb{G}_{1}^{t \times(t+1)}$ are computationally indistinguishable.

Lemma 2 Let $\mathbf{a}, \mathbf{b} \stackrel{\$}{\leftarrow} \mathbb{Z}_{p}^{t}, c \stackrel{\$}{\leftarrow} \mathbb{Z}_{p}^{t \times t}$ be chosen uniformly at random. If the XDDH assumption is $\epsilon^{\prime}$-hard in $\mathbb{G}_{1}$, then for any PPT B it holds $\mid \operatorname{Pr}\left[\mathcal{B}\left(g_{1}^{\mathbf{a}}, g_{1}^{\mathbf{a} \cdot \mathbf{b}^{\top}}\right)=1\right]-\operatorname{Pr}\left[\mathcal{B}\left(g_{1}^{\mathbf{a}}, g_{1}^{c}\right)=\right.$ $1] \mid \leq T \cdot \epsilon^{\prime}$.

We first show how to use Lemma 2 to prove that $\mathrm{H}_{\text {sqrt }}$ has programmable pseudorandomness. The proof of Lemma 2 appears slightly below.

Let $\mathcal{A}$ be an adversary that breaks the $\epsilon$-programmable pseudorandomness of $\mathrm{H}_{\text {sqrt }}$. We construct a simulator $\mathcal{B}$ that can distinguish the two distributions $\left(g_{1}^{\mathbf{a}}, g_{1}^{\mathbf{a} \cdot \mathbf{b}^{\top}}\right)$ and $\left(g_{1}^{\mathbf{a}}, g_{1}^{c}\right)$ described above with advantage greater than $\epsilon$.

$\mathcal{B}$ 's input is a tuple $\left(\mathbf{A}^{\prime}, C\right) \in \mathbb{G}_{1}^{t} \times \mathbb{G}_{1}^{t \times t}$ and its goal is to decide about the distribution of $C$. First, $\mathcal{B}$ runs $\mathcal{A}(\mathrm{bgp})$ which outputs the generators $h_{1}, h_{2} . \mathcal{B}$ then samples two random vectors $\mathbf{r}, \boldsymbol{\beta} \stackrel{\$}{\leftarrow} \mathbb{Z}_{p}^{t}$, computes $\mathbf{B}=g_{2}^{\boldsymbol{\beta}} \in \mathbb{G}_{2}^{t}, \mathbf{A}=h_{1}^{\mathbf{r}} \cdot \mathbf{A}^{\prime} \in \mathbb{G}_{1}^{t}$, sets pek $=(\mathbf{A}, \mathbf{B})$, and runs $\mathcal{A}$ (pek) Next, for every oracle query $(i, j)$ made by $\mathcal{A}, \mathcal{B}$ simulates the answer by returning to $\mathcal{A}: \mathrm{H}(i, j)=A_{i}^{\beta_{j}}$ and $C_{i, j}$. It is easy to see that if $C=g_{1}^{\mathbf{a} \cdot \mathbf{b}^{\top}}$ then $\mathcal{B}$ is perfectly simulating $\operatorname{Exp}_{\mathcal{A}, \mathrm{H}_{\text {sqrt }}}^{P R H-0}$, otherwise, if $C$ is random and independent, then $\mathcal{B}$ is simulating $\operatorname{Exp}_{\mathcal{A}, H_{\text {sqrt }}}^{P R H-1}$. As a final note, we observe that the above proof works even in the case $h_{1}=1$.

Proof (Proof of Lemma 2) To prove the above lemma, we define $T+1$ hybrid distributions as follows. Let $\mathbf{a}, \mathbf{b} \stackrel{\$}{\leftarrow} \mathbb{Z}_{p}^{t}$ and $c \stackrel{\$}{\leftarrow} \mathbb{Z}_{p}^{t \times t}$ be randomly chosen. For every $0 \leq k \leq T$ we define the matrix $\mathcal{M}_{k} \in \mathbb{G}_{1}^{t \times t}$ by specifying the value $\mathcal{M}_{k}[i, j]$ of each entry $(i, j) \in[t] \times[t]$ of the matrix. For every $k^{\prime} \in[T]$, let $(i, j) \in[t] \times[t]$ be such that $k^{\prime}=j+(i-1) t$. Then:

- If $k^{\prime} \leq k, \mathcal{M}_{k}[i, j]=g_{1}^{c_{i, j}}$,

- If $k^{\prime}>k, \mathcal{M}_{k}[i, j]=g_{1}^{a_{i} b_{j}}$.

Notice that $\mathcal{M}_{0}=g_{1}^{\mathbf{a} \cdot \mathbf{b}^{\top}}$ while $\mathcal{M}_{T}=g_{1}^{c}$. Moreover,

$$
\begin{aligned}
& \left|\operatorname{Pr}\left[\mathcal{B}\left(g_{1}^{\mathbf{a}}, \mathcal{M}_{0}\right)=1\right]-\operatorname{Pr}\left[\mathcal{B}\left(g_{1}^{\mathbf{a}}, \mathcal{M}_{T}\right)=1\right]\right| \\
& \quad \leq \sum_{k=1}^{T}\left|\operatorname{Pr}\left[\mathcal{B}\left(g_{1}^{\mathbf{a}}, \mathcal{M}_{k-1}\right)=1\right]-\operatorname{Pr}\left[\mathcal{B}\left(g_{1}^{\mathbf{a}}, \mathcal{M}_{k}\right)=1\right]\right| \\
& \quad \leq T \cdot\left|\operatorname{Pr}\left[\mathcal{B}\left(g_{1}^{\mathbf{a}}, \mathcal{M}_{k-1}\right)=1\right]-\operatorname{Pr}\left[\mathcal{B}\left(g_{1}^{\mathbf{a}}, \mathcal{M}_{k}\right)=1\right]\right|
\end{aligned}
$$

We complete the proof of Lemma 2 by showing the following claim:

Claim 1 For every $1 \leq k \leq T$, if XDDH is $\epsilon^{\prime}$-hard in $\mathbb{G}_{1}$, then

$$
\mid \operatorname{Pr}\left[\mathcal{B}\left(g_{1}^{\mathbf{a}}, \mathcal{M}_{k-1}=1\right]-\operatorname{Pr}\left[\mathcal{B}\left(g_{1}^{\mathbf{a}}, \mathcal{M}_{k}\right)=1\right] \mid \leq \epsilon^{\prime}\right.
$$


Assume by contradiction that $\mid \operatorname{Pr}\left[\mathcal{B}\left(g_{1}^{\mathbf{a}}, \mathcal{M}_{k-1}=1\right]-\operatorname{Pr}\left[\mathcal{B}\left(g_{1}^{\mathbf{a}}, \mathcal{M}_{k}\right)=1\right] \mid \geq \epsilon^{\prime}\right.$. Then it is possible to build a simulator $\mathcal{B}^{\prime}$ which breaks the XDDH assumption in $\mathbb{G}_{1}$ with advantage greater than $\epsilon^{\prime} . \mathcal{B}^{\prime}$ gets an XDDH instance $\left(g_{1}, g_{1}^{\alpha}, g_{1}^{\beta}, g_{1}^{\gamma}\right)$ and proceeds as follows:

- It samples $c_{1}, \ldots, c_{k-1} \stackrel{\$}{\leftarrow} \mathbb{Z}_{p}$.

- It samples $a_{1}, \ldots, a_{\hat{i}-1}, a_{\hat{i}+1}, \ldots, a_{t}, b_{1}, \ldots, b_{\hat{j}-1}, b_{\hat{j}+1}, \ldots, b_{t} \stackrel{\$}{\leftarrow} \mathbb{Z}_{p}$, where $(\hat{i}, \hat{j})$ correspond to $k$, i.e., $k=\hat{j}+(\hat{i}-1) t$.

- It implicitly sets $\tilde{\mathbf{a}}=\left(a_{1}, \ldots, a_{\hat{i}-1}, \alpha, a_{\hat{i}+1}, \ldots a_{t}\right)$ and $\tilde{\mathbf{b}}=\left(b_{1}, \ldots, b_{\hat{j}-1}, \beta\right.$, $\left.b_{\hat{j}+1}, \ldots b_{t}\right)$

- $\mathcal{B}^{\prime}$ builds a matrix $\mathcal{M} \in \mathbb{G}_{1}^{t \times t}$ where:

- If $k^{\prime} \leq k-1, \mathcal{M}[i, j]=g_{1}^{c_{i, j}}$,

- If $k^{\prime}=k, \mathcal{M}[i, j]=g_{1}^{\gamma}$,

- If $k^{\prime}>k, \mathcal{M}[i, j]=g_{1}^{\tilde{a}_{i} \cdot \tilde{b}_{j}}$. Notice that such value can be efficiently computed by $\mathcal{B}^{\prime}$ as it knows $g_{1}^{\tilde{a}_{\hat{i}}}=g_{1}^{\alpha}, g_{1}^{\tilde{b}_{\hat{j}}}=g_{1}^{\beta}, \tilde{a}_{i}, \forall i \neq \hat{i}, \tilde{b}_{j}, \forall j \neq \hat{j}$, and $k^{\prime}>k$ implies $(i, j) \neq(\hat{i}, \hat{j})$.

- $\mathcal{B}^{\prime}$ runs $b^{\prime} \leftarrow \mathcal{B}\left(g_{1}^{\tilde{\mathbf{a}}}, \mathcal{M}\right)$ and returns the same bit $b^{\prime}$.

As one can check, if $\gamma=\alpha \beta$, then $\mathcal{M}^{\prime}$ is distributed as $\mathcal{M}_{k-1}$. Otherwise, if $\gamma$ is random and independent, $\mathcal{M}^{\prime}$ is distributed as $\mathcal{M}_{k}$. Therefore,

$$
\begin{gathered}
\left|\operatorname{Pr}\left[\mathcal{B}^{\prime}\left(g_{1}, g_{1}^{\alpha}, g_{1}^{\beta}, g_{1}^{\alpha \beta}\right)=1\right]-\operatorname{Pr}\left[\mathcal{B}^{\prime}\left(g_{1}, g_{1}^{\alpha}, g_{1}^{\beta}, g_{1}^{\gamma}\right)=1\right]\right| \\
=\left|\operatorname{Pr}\left[\mathcal{B}\left(g_{1}^{\tilde{\mathbf{a}}}, \mathcal{M}_{k-1}\right)=1\right]-\operatorname{Pr}\left[\mathcal{B}\left(g_{1}^{\tilde{\mathbf{a}}}, \tilde{\mathcal{M}}_{k}\right)=1\right]\right| \geq \epsilon^{\prime}
\end{gathered}
$$

(poly, 0, 2)-programmability of $\mathrm{H}_{\text {sqrt }}$ Below we show that $\mathrm{H}_{\text {sqrt }}$ is (poly, 0, 2, $\gamma, \delta$ )programmable for $\gamma=0$ and $\delta=1$. While such (poly, 0 )-programmability might look uninteresting at first, this property turns out to be useful in various security proofs, as shown in our application to homomorphic signatures of Sect. 4.

Theorem 5 The asymmetric hash function $\mathrm{H}_{\mathrm{sqrt}}$ described above is (poly, 0,d, $\gamma, \delta$ )programmable with $d=2, \gamma=0$ and $\delta=1$. Furthermore, in the case when either $\hat{h}_{1}=\hat{g}_{1}$ or $\hat{h}_{2}=\hat{g}_{2}, \mathrm{H}_{\mathrm{sqr}}$ is (poly, $0, d, \gamma, \delta$ )-programmable with $d=1, \gamma=0$ and $\delta=1$.

Proof The trapdoor algorithms are defined as follows:

H.TrapGen $\left(1^{\lambda}, \hat{g}_{1}, \hat{h}_{1}, \hat{g}_{2}, \hat{h}_{2}\right)$ : first, sample $r_{i}, s_{i} \stackrel{\$}{\leftarrow} \mathbb{Z}_{p}$ for all $i \in[t]$ and then set $A_{i}=\hat{h}_{1}^{r_{i}}, B_{i}=\hat{h}_{2}^{s_{i}}$. Finally, set td $=\left(\left\{r_{i}, s_{i}\right\}_{i=1}^{t}\right)$, pek $=\left\{A_{i}, B_{i}\right\}_{i=1}^{t}$, and output (td, pek).

H. TrapEval(td, $X)$ : let $X=(i, j)$, and then return the coefficients of the degree-2 polynomial

$$
c_{X}\left(y_{1}, y_{2}\right)=\left(y_{1} y_{2}\right) r_{i} s_{j}
$$

Syntax and correctness are easily seen by inspection. The public key generated by $\mathrm{H}$. TrapGen is distributed identically to the one generated by H.Gen, from which $\gamma=0$. Also, it is clear that for any $X \in \mathcal{X}$, the degree- 0 term of the polynomial $c_{X}$ computed by $\mathrm{H}$. TrapEval is always 0 . It is straightforward to see that in the case $y_{1}=1$ (or $y_{2}=1$ ) the function satisfies the programmability with $d=1$. 
Weak (poly, 1,2)-programmability of $\mathrm{H}_{\text {sqrt }}$ Here we prove that $\mathrm{H}_{\text {sqrt }}$ is weakly (poly, 1,2, $\gamma, \delta$ )-programmable for $\gamma=0$ and $\delta=1$.

Theorem 6 The asymmetric hash function $\mathrm{H}_{\text {sqrt }}$ described above is weakly (poly, $1, d, \gamma, \delta$ )programmable with $d=2, \gamma=0$ and $\delta=1$.

Proof The trapdoor algorithms are defined as follows:

H.TrapGen $\left(1^{\lambda}, \hat{g}_{1}, \hat{h}_{1}, \hat{g}_{2}, \hat{h}_{2}, Z\right): \quad$ let $Z=\left(i^{*}, j^{*}\right) \in[t] \times[t]$. First, sample $r_{i}, s_{i} \stackrel{\$}{\leftarrow} \mathbb{Z}_{p}$ for all $i \in[t]$. Next, compute $A_{i^{*}}=\hat{g}_{1} \hat{h}_{1}^{r_{i}}, B_{j^{*}}=$ $\hat{g}_{2} \hat{h}_{2}^{S^{*}}, A_{i}=\hat{h}_{1}^{r_{i}}, \forall i \neq i^{*}$ and $B_{j}=\hat{h}_{2}^{S_{j}}, \forall j \neq j^{*}$. Finally, set td $=\left(\left\{r_{i}, s_{i}\right\}_{i=1}^{t}\right)$, pek $=\left\{A_{i}, B_{i}\right\}_{i=1}^{t}$, and output (td, pek).

H. TrapEval (td, $X)$ : given $X=(i, j)$, return the coefficients of the degree2 polynomial

$$
c_{X}\left(y_{1}, y_{2}\right)=\alpha_{i}\left(y_{1}\right) \cdot \beta_{j}\left(y_{2}\right)
$$

where $\alpha_{i}\left(y_{1}\right)$ (resp. $\beta_{j}\left(y_{2}\right)$ ) is the discrete logarithm of $A_{i}$ (resp. $B_{j}$ ) in base $\hat{g}_{1}$ (resp. $\hat{g}_{2}$ ), viewed as a degree-1 polynomial in the unknown $y_{1}$ (resp. $y_{2}$ ).

Syntax and correctness are easily seen by inspection. The public key generated by $\mathrm{H}$. TrapGen is distributed identically to the one generated by H.Gen, from which $\gamma=0$. Also, it is clear from the construction that for $Z=\left(i^{*}, j^{*}\right)$ we have $c_{Z}\left(y_{1}, y_{2}\right)=\left(y_{1} r_{1}+1\right)\left(y_{2} s_{j}+1\right)$, and thus $c_{Z, 0}=1$, whereas for every $X \neq Z$ the degree- 0 term of the polynomial $c_{Z}\left(y_{1}, y_{2}\right)$ computed by $\mathrm{H}$. TrapEval is always 0 . And this holds with probability $\delta=1$.

Weak (poly, 1, 2)-degree-2-programmability of $\mathrm{H}_{\text {sqrt }}$ Finally, we prove that $\mathrm{H}_{\text {sqrt }}$ is also weakly (poly, 1, 2, $\gamma, \delta$ )-degree-2-programmable for $\gamma=0$ and $\delta=1$.

Theorem 7 The asymmetric hash function $\mathrm{H}_{\text {sqrt }}$ described above is weakly (poly, $1, d, \gamma, \delta$ )degree-2 programmable with $d=2, \gamma=0$ and $\delta=1$.

Proof The proof of this theorem can be seen as the "dual" version of the one of Theorem 6. Instead of setting the simulated keys so that $Z$ is the only input for which $c_{Z, 0}=1$, here the keys are simulated in such a way that $Z$ is the only input in which the term $y_{1} y_{2}$ appears. More precisely, the trapdoor algorithms work as follows:

H.TrapGen $\left(1^{\lambda}, \hat{g}_{1}, \hat{h}_{1}, \hat{g}_{2}, \hat{h}_{2}, Z\right): \quad$ let $Z=\left(i^{*}, j^{*}\right) \in[t] \times[t]$. First, sample $r_{i}, s_{i} \stackrel{\$}{\leftarrow}$ $\mathbb{Z}_{p}$ for all $i \in[t]$ and then set $A_{i^{*}}=\hat{h}_{1} \hat{g}_{1}^{r_{i}}, B_{j^{*}}=$ $\hat{h}_{2} \hat{g}_{2}^{S_{j^{*}}}, A_{i}=\hat{g}_{1}^{r_{i}}, \forall i \neq i^{*}$ and $B_{j}=\hat{g}_{2}^{s_{j}}, \forall j \neq j^{*}$. Finally, set td $=\left(\left\{r_{i}, s_{i}\right\}_{i=1}^{t}\right)$, pek $=\left\{A_{i}, B_{i}\right\}_{i=1}^{t}$, and output (td, pek).

H.TrapEval (td, $X)$ : let $X=(i, j)$, and then return the coefficients of the degree-2 polynomial

$$
c_{X}\left(y_{1}, y_{2}\right)=\alpha_{i}\left(y_{1}\right) \cdot \beta_{j}\left(y_{2}\right)
$$

where $\alpha_{i}\left(y_{1}\right)$ (resp. $\beta_{j}\left(y_{2}\right)$ ) is the discrete logarithm of $A_{i}$ (resp. $B_{j}$ ) in base $\hat{g}_{1}$ (resp. $\hat{g}_{2}$ ), viewed as a degree-1 polynomial in the unknown $y_{1}$ (resp. $y_{2}$ ). 
Syntax and correctness are easily seen by inspection. The public key generated by $\mathrm{H}$. TrapGen is distributed identically to the one generated by $\mathrm{H}$.Gen, from which $\gamma=0$. By construction, we have that for $Z=\left(i^{*}, j^{*}\right), c_{Z}\left(y_{1}, y_{2}\right)=\left(r_{1}+y_{1}\right)\left(s_{j}+y_{2}\right)$, and thus $c_{Z, 2}=1$, whereas for every $X \neq Z$ the polynomial $c_{X}\left(y_{1}, y_{2}\right)$ has degree $\leq 1$, and thus $c_{X, 2}=0$. This property holds with probability $\delta=1$.

\section{Linearly-homomorphic signatures with short public keys}

In this section, we show a new linearly-homomorphic signature scheme that uses APHFs in a generic way. By instantiating the APHFs with our construction $\mathrm{H}_{\text {sqrt }}$ given in Sect. 3, we obtain the first linearly-homomorphic signature scheme that is secure in the standard model, and whose public key has a size that is sub-linear in both the dataset size and the dimension of the signed vectors. Precisely, if the signature scheme supports datasets of maximal size $N$ and can sign vectors of dimension $T$, then the public key of our scheme is of size $O(\sqrt{N}+\sqrt{T})$. All previously existing constructions in the standard model achieved only public keys of length $O(N+T)$. Furthermore, our scheme is adaptive secure and achieves the interesting property of efficient verification that allows to use the scheme for verifiable delegation of computation in the preprocessing model [20].

Before describing our scheme, in the next section we recall the definition of homomorphic signatures.

\subsection{Homomorphic signatures for multi-labeled programs}

In this section we recall the definition of homomorphic signatures as presented in [20]. This definition extends the one by Freeman in [22] in order to work with the general notion of multi-labeled programs [6,25].

Multi-labeled programs A labeled program $\mathcal{P}$ is a tuple $\left(f, \tau_{1}, \ldots, \tau_{n}\right)$ such that $f$ : $\mathcal{M}^{n} \rightarrow \mathcal{M}$ is a function of $n$ variables (e.g., a circuit) and $\tau_{i} \in\{0,1\}^{*}$ is a label of the $i$-th input of $f$. Labeled programs can be composed as follows: given $\mathcal{P}_{1}, \ldots, \mathcal{P}_{t}$ and a function $g: \mathcal{M}^{t} \rightarrow \mathcal{M}$, the composed program $\mathcal{P}^{*}$ is the one obtained by evaluating $g$ on the outputs of $\mathcal{P}_{1}, \ldots, \mathcal{P}_{t}$, and it is denoted as $\mathcal{P}^{*}=g\left(\mathcal{P}_{1}, \ldots, \mathcal{P}_{t}\right)$. The labeled inputs of $\mathcal{P}^{*}$ are all the distinct labeled inputs of $\mathcal{P}_{1}, \ldots \mathcal{P}_{t}$ (all the inputs with the same label are grouped together and considered as a unique input of $\mathcal{P}^{*}$ ).

Let $f_{i d}: \mathcal{M} \rightarrow \mathcal{M}$ be the identity function and $\tau \in\{0,1\}^{*}$ be any label. We refer to $\mathcal{I}_{\tau}=\left(f_{i d}, \tau\right)$ as the identity program with label $\tau$. Note that a program $\mathcal{P}=\left(f, \tau_{1}, \ldots, \tau_{n}\right)$ can be expressed as the composition of $n$ identity programs $\mathcal{P}=f\left(\mathcal{I}_{\tau_{1}}, \ldots, \mathcal{I}_{\tau_{n}}\right)$.

A multi-labeled program $\mathcal{P}_{\Delta}$ is a pair $(\mathcal{P}, \Delta)$ in which $\mathcal{P}=\left(f, \tau_{1}, \ldots, \tau_{n}\right)$ is a labeled program while $\Delta \in\{0,1\}^{*}$ is a data set identifier. Multi-labeled programs can be composed within the same data set in the most natural way: given $\left(\mathcal{P}_{1}, \Delta\right), \ldots,\left(\mathcal{P}_{t}, \Delta\right)$ which has the same data set identifier $\Delta$, and given a function $g: \mathcal{M}^{t} \rightarrow \mathcal{M}$, the composed multi-labeled program $\mathcal{P}_{\Delta}^{*}$ is the pair $\left(\mathcal{P}^{*}, \Delta\right)$ where $\mathcal{P}^{*}$ is the composed program $g\left(\mathcal{P}_{1}, \ldots, \mathcal{P}_{t}\right)$, and $\Delta$ is the common data set identifier for all the $\mathcal{P}_{i}$. As for labeled programs, one can define the notion of a multi-labeled identity program as $\mathcal{I}_{(\Delta, \tau)}=\left(\left(f_{i d}, \tau\right), \Delta\right)$.

Definition 8 (Homomorphic signatures) A homomorphic signature scheme HSig consists of a tuple of PPT algorithms (KeyGen, Sign, Ver, Eval) satisfying the following four properties: authentication correctness, evaluation correctness, succinctness and security. The four algorithms work as follows: 
the key generation algorithm takes as input a security parameter $\lambda$, the description of the label space $\mathcal{L}$ (which fixes the maximum data set size $N$ ), and outputs a public key vk and a secret key sk. The public key vk defines implicitly a message space $\mathcal{M}$ and a set $\mathcal{F}$ of admissible functions.

Sign(sk, $\Delta, \tau, m)$ the signing algorithm takes as input a secret key sk, a data set identifier $\Delta$, a label $\tau \in \mathcal{L}$ a message $m \in \mathcal{M}$, and it outputs a signature $\sigma$.

$\operatorname{Ver}\left(\mathrm{vk}, \mathcal{P}_{\Delta}, m, \sigma\right)$ the verification algorithm takes as input a public key vk, a multilabeled program $\mathcal{P}_{\Delta}=\left(\left(f, \tau_{1}, \ldots, \tau_{n}\right), \Delta\right)$ with $f \in \mathcal{F}$, a message $m \in \mathcal{M}$, and a signature $\sigma$. It outputs either 0 (reject) or 1 (accept).

Eval $(\mathrm{vk}, f, \sigma) \quad$ the evaluation algorithm takes as input a public $\mathrm{vk}$, a function $f \in \mathcal{F}$ and a tuple of signatures $\left\{\sigma_{i}\right\}_{i=1}^{n}$ (assuming that $f$ takes $n$ inputs). It outputs a new signature $\sigma$.

Below we describe the four properties mentioned above:

Authentication correctness Intuitively, a homomorphic signature scheme has authentication correctness if the signature generated by $\operatorname{Sign}(\mathrm{sk}, \Delta, \tau, m$ ) verify correctly for $m$ as the output of the identity program $\mathcal{I}_{\Delta, \tau}$. More formally, the scheme HSig satisfies the authentication correctness property if for a given label space $\mathcal{L}$, all key pairs (sk, vk) $\leftarrow \operatorname{KeyGen}\left(1^{\lambda}, \mathcal{L}\right)$, any label $\tau \in \mathcal{L}$, data identifier $\Delta \in\{0,1\}^{*}$, and any signature $\sigma \leftarrow \operatorname{Sign}(\mathrm{sk}, \Delta, \tau, m)$, Ver(vk, $\left.\mathcal{I}_{\Delta, \tau}, m, \sigma\right)$ outputs 1 with all but negligible probability.

Evaluation correctness Intuitively, this property says that running the evaluation algorithm on signatures $\left(\sigma_{1}, \ldots, \sigma_{n}\right)$ such that each $\sigma_{i}$ verifies for $m_{i}$ as the output of a multi-labeled program $\left(P_{i}, \Delta\right)$, produces a signature $\sigma$ which verifies for $f\left(m_{1}, \ldots, m_{t}\right)$ as the output of the composed program $\left(f\left(\mathcal{P}_{1}, \ldots, \mathcal{P}_{n}\right), \Delta\right)$. More formally, fix a key pair (vk, sk $) \stackrel{\$}{\leftarrow}$ KeyGen $\left(1^{\lambda}, \mathcal{L}\right)$, a function $g: \mathcal{M}^{t} \rightarrow \mathcal{M}$, and any set of program/message/signature triples $\left\{\left(\mathcal{P}_{i}, m_{i}, \sigma_{i}\right)\right\}_{i=1}^{t}$ such that $\operatorname{Ver}\left(\mathrm{Vk}, \mathcal{P}_{i}, m_{i}, \sigma_{i}\right)=1$. If $m^{*}=g\left(m_{1}, \ldots, m_{t}\right), \mathcal{P}^{*}=$ $g\left(\mathcal{P}_{1}, \ldots, \mathcal{P}_{t}\right)$, and $\sigma^{*}=\operatorname{Eval}\left(\mathrm{vk}, g,\left(\sigma_{1}, \ldots, \sigma_{t}\right)\right)$, then $\operatorname{Ver}\left(\mathrm{vk}, \mathcal{P}^{*}, m^{*}, \sigma^{*}\right)=1$ holds with all but negligible probability.

Succintness A homomorphic signature scheme is said to be succint if, for a fixed security parameter $\lambda$, the size of signatures depends at most logarithmically on the data set size $N$.

Security To define the security notion of homomorphic signatures we define the following experiment HomUF-CMA ${ }_{\mathcal{A}, \text { HomSign }}(\lambda)$ between an adversary $\mathcal{A}$ and a challenger $\mathcal{C}$ :

Key generation $\mathcal{C}$ runs $(\mathrm{vk}, \mathrm{sk}) \stackrel{\$}{\leftarrow} \operatorname{KeyGen}\left(1^{\lambda}, \mathcal{L}\right)$ and gives vk to $\mathcal{A}$.

Signing queries $\mathcal{A}$ can adaptively submit queries of the form $(\Delta, \tau, m)$, where $\Delta$ is a data set identifier, $\tau \in \mathcal{L}$, and $m \in \mathcal{M}$. The challenger $\mathcal{C}$ proceeds as follows: if $(\Delta, \tau, m)$ is the first query with the data set identifier $\Delta$, the challenger initializes an empty list $T_{\Delta}=\emptyset$ for $\Delta$. If $T_{\Delta}$ does not already contain a tuple $(\tau, \cdot)$ (which means that $\mathcal{A}$ never asked for a query $(\Delta, \tau, \cdot))$, the challenger $\mathcal{C}$ computes $\sigma \stackrel{S}{\leftarrow} \operatorname{Sign}(\mathrm{sk}, \Delta, \tau, m)$, returns $\sigma$ to $\mathcal{A}$ and updates the list $T_{\Delta} \leftarrow T_{\Delta} \cup(\tau, m)$. If $(\tau, m) \in$ $T_{\Delta}$ (which means that the adversary had already queried the tuple $(\Delta, \tau, m))$, then $\mathcal{C}$ replies with the same signature generated before. If $T_{\Delta}$ contains a tuple $\left(\tau, m^{\prime}\right)$ for some message $m^{\prime} \neq m$, then the challenger ignores the query.

Forgery At the end $\mathcal{A}$ outputs a tuple $\left(\mathcal{P}_{\Delta^{*}}^{*}, m^{*}, \sigma^{*}\right)$.

The experiment HomUF-CMA $A_{\mathcal{A}, \text { HomSign }}(\lambda)$ outputs 1 if the tuple returned by $\mathcal{A}$ is a forgery, and 0 otherwise. 
To define what is a forgery in such a game we recall the notion of well defined program with respect to a list $T_{\Delta}[20]$.

Definition 9 A labeled program $\mathcal{P}^{*}=\left(f^{*}, \tau_{1}^{*}, \ldots, \tau_{n}^{*}\right)$ is well defined with respect to $T_{\Delta^{*}}$ if one of the two following cases holds:

- $\exists m_{1}, \ldots, m_{n}$ s.t. $\left(\tau_{i}^{*}, m_{i}\right) \in T_{\Delta^{*}} \forall i=1, \ldots, n$.

$-\exists i \in\{1, \ldots, n\}$ s.t. $\left(\tau_{i}, \cdot\right) \notin T_{\Delta^{*}}$ and $f^{*}\left(\left\{m_{j}\right\}_{\left(\tau_{j}, m_{j}\right) \in T_{\Delta^{*}}} \cup\left\{\tilde{m}_{\left(\tau_{j},\right) \notin T_{\Delta^{*}}}\right\}\right)$ does not change for all possible choices of $\tilde{m}_{j} \in \mathcal{M}$.

Intuitively, the first case says that the challenger has generated signatures for the entire input space of $f$ for the data set $\Delta^{*}$, while the second one means that the inputs that were not signed during the experiment do not contribute to the result of $f$.

Using this notion, it is then possible to define the three different types of forgeries that can occur in the experiment HomUF-CMA:

Type 1: $\operatorname{Ver}\left(\mathrm{vk}, \mathcal{P}_{\Delta^{*}}^{*}, m^{*}, \sigma^{*}\right)=1$ and the list $T_{\Delta^{*}}$ was not initialized during the game (i.e., no message was ever signed w.r.t. data set identifier $\Delta^{*}$ ).

Type 2: $\operatorname{Ver}\left(\mathrm{vk}, \mathcal{P}_{\Delta^{*}}^{*}, m^{*}, \sigma^{*}\right)=1, \mathcal{P}^{*}$ is well defined with respect to $T_{\Delta^{*}}$ and $m^{*} \neq$ $f^{*}\left(\left\{m_{j}\right\}_{\left(\tau_{j}, m_{j}\right) \in T_{\Delta^{*}}}\right)$ (i.e., $m^{*}$ is not the correct output of $\mathcal{P}^{*}$ when executed over previously signed messages).

Type 3: $\operatorname{Ver}\left(\mathrm{vk}, \mathcal{P}_{\Delta^{*}}^{*}, m^{*}, \sigma^{*}\right)=1$ and $\mathcal{P}^{*}$ is not well defined with respect to $T_{\Delta^{*}}$.

Then we say that HSig is a secure homomorphic signature if for any PPT adversary $\mathcal{A}$, we have that $\operatorname{Pr}\left[\operatorname{Hom} U F-C M A_{\mathcal{A}, \operatorname{HomSign}}(\lambda)=1\right] \leq \epsilon(\lambda)$ where $\epsilon(\lambda)$ is a negligible function.

We recall that, as proved by Freeman in [22], in a linearly-homomorphic signature scheme any adversary who outputs a Type 3 forgery can be converted into one that outputs a Type 2 one.

Proposition 1 ([22]) Let $\mathrm{HSig}$ be a linearly homomorphic signature scheme with message space $\mathcal{M} \subset \mathcal{R}^{n}$ for some ring $\mathcal{R}$. If $\mathrm{HSig}$ is secure against Type 2 forgeries, then $\mathrm{HSig}$ is secure against Type 3 forgeries.

Homomorphic signatures with efficient verification We recall the notion of homomorphic signatures with efficient verification introduced in [20]. The property states that the verification algorithm can be split in two phases: an offline phase where, given the verification key $v k$ and a labeled program $\mathcal{P}$, one precomputes a concise key $\mathrm{vk}_{\mathcal{P}}$; an online phase in which $v k_{\mathcal{P}}$ can be used to verify signatures w.r.t. $\mathcal{P}$ and any dataset $\Delta$. To achieve (amortized) efficiency, the idea is that $\mathbf{v} \mathbf{k}_{\mathcal{P}}$ can be reused an unbounded number of times, and the online verification is cheaper than running $\mathcal{P}$. Below is the formal definition.

Definition 10 Let HSig = (KeyGen, Sign, Ver, Eval) be a homomorphic signature scheme for multi-labeled programs. HSig satisfies efficient verification if there exist two additional algorithms (VerPrep, EffVer) such that:

VerPrep $(\mathbf{v k}, \mathcal{P})$ :

on input the verification key $\mathrm{vk}$ and a labeled program $\mathcal{P}=$ $\left(f, \tau_{1}, \ldots, \tau_{n}\right)$, this algorithm generates a concise verification key $\mathrm{vk}_{\mathcal{P}}$. We stress that this verification key does not depend on any data set identifier $\Delta$.

$\operatorname{EffVer}\left(\mathrm{vk}_{\mathcal{P}}, \Delta, m, \sigma\right)$ : given a verification key $\mathrm{vk}_{\mathcal{P}}$, a data set identifier $\Delta$, a message $m \in \mathcal{M}$ and a signature $\sigma$, the efficient verification algorithm outputs 0 (reject) or 1 (accept). 
The above algorithms are required to satisfy the following two properties:

Correctness Let (sk, vk) $\stackrel{\$}{\leftarrow}$ KeyGen $\left(1^{\lambda}\right)$ be honestly generated keys, and $\left(\mathcal{P}_{\Delta}, m, \sigma\right)$ be any program/message/signature tuple with $\mathcal{P}_{\Delta}=(\mathcal{P}, \Delta)$ such that $\operatorname{Ver}\left(\mathrm{vk}, \mathcal{P}_{\Delta}, m, \sigma\right)=1$. Then, for every $\mathrm{vk}_{\mathcal{P}} \stackrel{\$}{\leftarrow} \operatorname{VerPrep}(\mathrm{vk}, \mathcal{P})$, EffVer $\left(\mathrm{vk}_{\mathcal{P}}, \Delta, m, \sigma\right)=1$ holds with all but negligible probability.

Amortized efficiency Let $\mathcal{P}_{\Delta}=(\mathcal{P}, \Delta)$ be a program, let $\left(m_{1}, \ldots, m_{n}\right) \in \mathcal{M}^{n}$ be any vector of inputs, and let $t(n)$ be the time required to compute $\mathcal{P}\left(m_{1}, \ldots, m_{n}\right)$. If $\mathrm{vk}_{\mathcal{P}} \leftarrow$ $\operatorname{VerPrep}(\mathrm{vk}, \mathcal{P})$, then the time required for $\operatorname{EffVer}\left(\mathrm{vk}_{\mathcal{P}}, \Delta, m, \tau\right)$ is $t^{\prime}=o(t(n))$.

Context-hiding secure homomorphic signatures We recall and formalize the notion of context-hiding homomorphic signature. Intuitively, it states that a signature which certifies $m$ as the output of a multi-labeled program $\mathcal{P}_{\Delta}=\left(\left(f, \tau_{1}, \ldots, \tau_{n}\right), \Delta\right)$ does not reveal anything about the underlying data beyond the result of the computation. We give a simulation-based notion of security, requiring that a signature $\sigma$ can be simulated given knowledge of only the labeled program $\mathcal{P}_{\Delta}$, its output $m$ and the secret key sk, but without $\mathcal{P}_{\Lambda}$ 's input. The simulated signature is required to be indistinguishable from one obtained by running the Eval algorithm to any distinguisher $\mathcal{D}$ that is also given a key pair (sk, vk), the label program $\mathcal{P}_{\Delta}$ and the signatures on the messages on which the program $\mathcal{P}_{\Delta}$ is evaluated. Essentially, this property says that a verifier, even with the knowledge of the secret key, cannot gain any information beyond what can be trivially inferred from the input of the verification algorithm.

Definition 11 An homomorphic signature scheme for multi-labeled programs supports context hiding if there exist additional PPT procedures $\tilde{\sigma} \leftarrow \operatorname{Hide}(\mathrm{vk}, m, \sigma)$ and HVerify $\left(\mathrm{vk}, \mathcal{P}_{\Delta}, m, \sigma\right)$ such that:

- Correctness: For any $(\mathrm{vk}, \mathrm{sk}) \leftarrow \operatorname{KeyGen}\left(1^{\lambda}, \mathcal{L}\right)$ and any tuple $\left(\mathcal{P}_{\Delta}, m, \sigma\right)$ such that $\operatorname{Ver}\left(\mathrm{vk}, \mathcal{P}_{\Delta}, m, \sigma\right)=1$, we have that if $\tilde{\sigma} \leftarrow \operatorname{Hide}(\mathrm{vk}, m, \sigma)$ then $\operatorname{HVerify}\left(\mathrm{vk}, \mathcal{P}_{\Delta}, m\right.$, $\tilde{\sigma})=1$.

- Unforgeability: The signature scheme is secure when we replace the original verification algorithm Ver with HVerify in the security game.

- Context-Hiding Security: There is a simulator Sim such that, for any fixed (worst-case) choice of $(\mathrm{sk}, \mathrm{vk}) \in \operatorname{KeyGen}\left(1^{\lambda}, \mathcal{L}\right)$, any labeled program $\mathcal{P}_{\Delta}=\left(f, \tau_{1}, \ldots, \tau_{\ell}, \Delta\right)$ and messages $m_{1}, \ldots, m_{\ell}$, there exists a function $\epsilon(\lambda)$ such that for any distinguisher $\mathcal{D}$ it holds

$$
\mid \operatorname{Pr}[\mathcal{D}(I, \operatorname{Hide}(\mathrm{vk}, m, \sigma))=1]-\operatorname{Pr}\left[\mathcal{D}\left(I, \operatorname{Sim}\left(\mathrm{sk}, \mathcal{P}_{\Delta}, m\right)=1\right] \mid=\epsilon(\lambda)\right.
$$

where $I=\left(\mathrm{sk}, \mathrm{vk}, \mathcal{P}_{\Delta},\left\{m_{i}, \sigma_{i}=\operatorname{Sign}\left(\mathrm{sk}, \Delta, \tau_{i}, m_{i}\right)\right\}_{i=1}^{\ell}\right), m=f\left(m_{1}, \ldots, m_{\ell}\right)$, $\sigma \leftarrow \operatorname{Eval}\left(\mathrm{vk}, \sigma_{1}, \ldots, \sigma_{\ell}\right)$, and the probabilities are taken over the randomness of Sign, Hide and Sim. If $\epsilon(\lambda)$ is negligible then the scheme is said to have statistical context-hiding, otherwise, if $\epsilon(\lambda)=0$, the scheme has perfect context-hiding.

Remark 2 (On the hiding algorithms) We would like to remark that the Hide procedure is introduced to aim for generality of the above definition. However an explicit Hide procedure may not be necessary, i.e., there may be schemes where the evaluation algorithm already produces context-hiding signatures. In these cases, the above definition still applies as the Hide procedure can be simply the identity function, and HVerify can be the regular verification algorithm Ver.

Remark 3 (Relation with existing definitions) As a second remark, we note that contexthiding security for homomorphic signatures has been considered in earlier works $[2,11,26]$ 
with (slightly) different definitions. Compared to the weakly context-hiding notion of Boneh and Freeman [11], ours is stronger in that it considers indistinguishability even when one knows the original signatures. The notion of Ahn et al. [2] is made for $P$-homomorphic signatures, where $P$ are predicates. Although $P$-homomorphic signatures and the homomorphic signatures considered in this work are equivalent (cf. [26, footnote 1]), with respect to context-hiding our notion is slightly weaker as it requires context-hiding to be satisfied with the help of specific hiding algorithms. However, it is not hard to see that when a scheme satisfies context-hiding with trivial hiding algorithms (i.e., Hide is the identity function and HVerify $=$ Ver), then it also satisfies the notion of [2]. Our definition above is inspired by that of [26] except for two main differences. First, in our case the simulator is explicitly given the circuit for which the signature is supposed to verify. This is in contrast to the definition in [26] where the simulator receives a value $\alpha$ output of a Process procedure, which can be seen as the equivalent of our VerPrep algorithm. With respect to this difference our definition is more general, and we stress that the circuit is not hidden in either of the two context-hiding notions. Second, our definition considers indistinguishability in the presence of the original signatures $\sigma_{i}$. Although including this information may not be necessary for schemes with a "powerful" hiding procedure, it allows for more generality. Finally, let us note that a disadvantage of using hiding algorithms is that after the application of Hide signatures may no longer be used in further homomorphic computation.

\subsection{Our construction}

Let $\Sigma^{\prime}=\left(\right.$ KeyGen', Sign', Ver') be a regular signature scheme, and $F: \mathcal{K} \times\{0,1\}^{*} \rightarrow$ $\mathbb{Z}_{p}$ be a pseudorandom function with key space $\mathcal{K}$. Our linearly-homomorphic signature scheme signs $T$-dimensional vectors of messages in $\mathbb{Z}_{p}$, supports datasets of size $N$, with both $N=\operatorname{poly}(\lambda)$ and $T=\operatorname{poly}(\lambda)$ and is context-hiding secure. Let $\mathrm{H}=(\mathrm{H}$.Gen, H.PriEval, H.PubEval) and $H^{\prime}=(H . G e n ', H . P r i E v a l ', H . P u b E v a l ')$ be two asymmetric programmable hash functions such that $\mathrm{H}:[N] \rightarrow \mathbb{G}_{1}$ and $\mathrm{H}^{\prime}:[T] \rightarrow \mathbb{G}_{1}$.

We construct a homomorphic signature HSig $=($ KeyGen, Sign, Ver, Eval) as follows:

KeyGen $\left(1^{\lambda}, \mathcal{L}, T\right)$. Let $\lambda$ be the security parameter, $\mathcal{L}$ be a set of admissible labels where $\mathcal{L}=\{1, \ldots, N\}$, and $T$ be an integer representing the dimension of the vectors to be signed. The key generation algorithm works as follows.

- Generate a key pair $\left(\mathbf{v k}^{\prime}, \mathbf{s k}^{\prime}\right) \stackrel{\$}{\leftarrow} \operatorname{KeyGen}\left(1^{\lambda}\right)$ for the regular scheme.

- Run bgp $\stackrel{\$}{\leftarrow} \mathcal{G}\left(1^{\lambda}\right)$ to generate the bilinear groups parameters bgp $=\left(p, \mathbb{G}_{1}, \mathbb{G}_{2}, \mathbb{G}_{T}\right.$, $\left.g_{1}, g_{2}, e\right)$ where $\mathbb{G}_{1}, \mathbb{G}_{2}$ and $\mathbb{G}_{T}$ are groups of prime order $p \approx 2^{\lambda}, g_{1} \in \mathbb{G}_{1}, g_{2} \in \mathbb{G}_{2}$ are generators and $e: \mathbb{G}_{1} \times \mathbb{G}_{2} \rightarrow \mathbb{G}_{T}$ is an efficiently computable, non-degenerate bilinear map.

- Choose two random seeds $K, \hat{K} \stackrel{\$}{\leftarrow} \mathcal{K}$ for the PRF $F: \mathcal{K} \times\{0,1\}^{*} \rightarrow \mathbb{Z}_{p}$.

- Run (sek, pek) $\stackrel{\$}{\leftarrow}$ H.Gen $\left(1^{\lambda}\right.$, bgp) and $\left(\right.$ sek $^{\prime}$, pek $\left.^{\prime}\right) \stackrel{\$}{\leftarrow}$ H.Gen $\left(1^{\lambda}\right.$, bgp) to generate the keys of the asymmetric hash functions.

- Return vk $=\left(\mathrm{vk}^{\prime}\right.$, bgp, pek, pek') and sk $=\left(\mathrm{sk}^{\prime}, K, \hat{K}\right.$, sek, sek' $)$.

$\operatorname{Sign}(\mathbf{s k}, \Delta, \tau, \mathbf{m})$. The signing algorithm takes as input the secret key sk, a data set identifier $\Delta \in\{0,1\}^{*}$, a label $\tau \in[N]$ and a message vector $\mathbf{m} \in \mathbb{Z}_{p}^{T}$, and proceeds as follows:

1. Derive the integer $z \leftarrow F_{K}(\Delta)$ using the PRF, and compute $Z=g_{2}^{z}$.

2. Compute $\sigma_{\Delta} \leftarrow \operatorname{Sign}^{\prime}\left(\mathrm{sk}^{\prime}, \Delta \mid Z\right)$ to bind $Z$ to the dataset identifier $\Delta$. 
3. Derive $r \leftarrow F_{\hat{K}}(\Delta \mid \tau)$, set $R=g_{1}^{r}$ and compute

$$
S=(\text { H.PriEval (sek, } \tau) \cdot R \cdot \prod_{j=1}^{T}{\text { H.PriEval' }\left(\text { sek }^{\prime}, j\right)^{m_{j}}}^{1 / z}
$$

4. Return a signature $\sigma=\left(\sigma_{\Delta}, Z, R, S\right)$.

Essentially, the algorithm consists of two main steps. First, it uses the PRF $F_{K}$ to derive a common parameter $z$ which is related to the data set $\Delta$, and it signs the public part, $Z=g_{2}^{z}$, of this parameter using the signature scheme $\Sigma^{\prime}$. Then it uses the same PRF $F_{\hat{K}}$ with a different seed $\hat{K}$ to create the pseudorandom element $R=g_{1} F_{\hat{K}}(\Delta \mid \tau)$. Second, it uses $z$ to create the homomorphic component $S$ of the signature, such that $S$ is now related to all $(\Delta, \tau, \mathbf{m})$.

$\operatorname{Eval}(\mathrm{vk}, f, \sigma)$.

The public evaluation algorithm takes as input the public key $\mathrm{vk}$, a linear function $f: \mathbb{Z}_{p}^{\ell} \rightarrow \mathbb{Z}_{p}$ described by its vector of coefficients $\mathbf{f}=\left(f_{1}, \ldots, f_{\ell}\right)$, and a vector $\sigma$ of $\ell$ signatures $\sigma_{1}, \ldots, \sigma_{\ell}$ where $\sigma_{i}=\left(\sigma_{\Delta, i}, Z_{i}, R_{i}, S_{i}\right)$ for $i=1, \ldots, \ell$. Eval returns a signature $\sigma=\left(\sigma_{\Delta}, Z, R, S\right)$ that is obtained by setting $Z=Z_{1}, \sigma_{\Delta}=\sigma_{\Delta, 1}$, and by computing

$$
R=\prod_{i=1}^{\ell} R_{i}^{f_{i}}, \quad S=\prod_{i=1}^{\ell} S_{i}^{f_{i}}
$$

$\operatorname{Ver}\left(\mathrm{vk}, \mathcal{P}_{\Delta}, \mathbf{m}, \sigma\right)$. Let $\mathcal{P}_{\Delta}=\left(\left(f, \tau_{1}, \ldots, \tau_{\ell}\right), \Delta\right)$ be a multi-labeled program such that $f: \mathbb{Z}_{p}^{\ell} \rightarrow \mathbb{Z}_{p}$ is a linear function described by coefficients $\mathbf{f}=$ $\left(f_{1}, \ldots, f_{\ell}\right)$. Let $\mathbf{m} \in \mathbb{Z}_{p}^{T}$ be a message-vector and $\sigma=\left(\sigma_{\Delta}, Z, R, S\right)$ be a signature.

First, run $\operatorname{Ver}^{\prime}\left(\mathrm{vk}^{\prime}, \Delta \mid Z, \sigma_{\Delta}\right)$ to check that $\sigma_{\Delta}$ is a valid signature for $Z$ and the dataset identifier $\Delta$ taken as input by the verification algorithm. If $\sigma_{\Delta}$ is not valid, stop and return 0 (reject).

Otherwise, output 1 if and only if the following equation is satisfied

$$
\begin{aligned}
e(S, Z)= & \left(\prod_{i=1}^{\ell} \text { H.PubEval }\left(\text { pek }, \tau_{i}\right)^{f_{i}}\right) \cdot e\left(R, g_{2}\right) \\
& \times\left(\prod_{j=1}^{T} \text { H.PubEval' }\left(\text { pek }^{\prime}, j\right)^{m_{j}}\right)
\end{aligned}
$$

Finally, we describe the algorithms for efficient verification:

$\operatorname{VerPrep}(\mathrm{vk}, \mathcal{P})$. Let $\mathcal{P}=\left(f, \tau_{1}, \ldots, \tau_{\ell}\right)$ be a labeled program for a linear function $f: \mathbb{Z}_{p}^{\ell} \rightarrow \mathbb{Z}_{p}$. The algorithm computes $H=$ $\prod_{i=1}^{\ell}$ H.PubEval (pek, $\left.\tau_{i}\right)^{f_{i}}$, and returns the concise verification key $\mathrm{vk}_{\mathcal{P}}=\left(\mathrm{vk}^{\prime}, \mathrm{bgp}, H\right.$, pek').

EffVer $\left(\operatorname{vk}_{\mathcal{P}}, \Delta, \mathbf{m}, \sigma\right)$. The online verification is the same as Ver except that in the verification equation the value $H$ has been already computed in the off-line phase (and is included in $\mathbf{v k}_{\mathcal{P}}$ ).

Clearly, running the combination of VerPrep and EffVer gives the same result as running Ver, and EffVer's running time is independent of $f$ 's complexity $\ell$. 
We formally show the correctness of our homomorphic signature scheme in Sect. 4.3. The following theorems state the security and context-hiding of our scheme. Their proofs appear in Sects. 4.4 and 4.5 respectively.

Theorem 8 (Unforgeability of HSig) Assume that $\Sigma^{\prime}$ is an unforgeable signature scheme, $F$ is a pseudorandom function, and $\mathcal{G}$ is a bilinear group generator such that: $\mathrm{H}$ has $(1, \gamma, \epsilon)$-programmable pseudorandomness; $H^{\prime}$ is weakly (poly, $1,2, \gamma^{\prime}, \delta^{\prime}$ )-programmable and (poly, 0, 1, $\gamma^{\prime}, \delta^{\prime}$ )-programmable; the 2-DHI and the FDHI assumptions hold. Then HSig is a secure linearly-homomorphic signature scheme.

We note that our scheme HSig can be instantiated by instantiating both $\mathrm{H}$ and $\mathrm{H}^{\prime}$ with two different instances of our programmable hash $\mathrm{H}_{\text {sqrt }}$ described in Sect. 3.3. As one can check in Sect. 3.3, $\mathrm{H}_{\text {sqrt }}$ allows for the multiple programmability modes required in our Theorem 8 . Let us stress that requiring the same function to have multiple programmability modes is not contradictory, as such modes do not have to hold simultaneously. It simply means that for the same function there exist different pairs of trapdoor algorithms each satisfying programmability with different parameters. ${ }^{5}$

Theorem 9 (Context-hiding of $\mathrm{HSig}$ ) Assume that $\Sigma^{\prime}$ is an unforgeable signature scheme, $F$ is a pseudorandom function, and $\mathrm{H}=(\mathrm{H} . \mathrm{Gen}, \mathrm{H}$.PriEval, $\mathrm{H} . P u b E v a l)$ and $\mathrm{H}^{\prime}=(\mathrm{H} . \mathrm{Gen}$, $\mathrm{H}$.PriEval', H.PubEval') be two asymmetric programmable hash functions such that $\mathrm{H}$ : $[N] \rightarrow \mathbb{G}_{1}$ and $\mathrm{H}^{\prime}:[T] \rightarrow \mathbb{G}_{1}$. If $\Sigma^{\prime}$ is deterministic, then the scheme $\mathrm{HSig}$ satisfies perfect context hiding in the sense of Definition 11.

We note that for the sake of context-hiding in our scheme there are no specific hiding algorithms (i.e., one can see Hide as the identity function and HVerify as Ver). In other words the signing and the evaluation algorithms already produce signatures with context-hiding.

Remark 4 (Alternative requirement for context-hiding) To prove context-hiding of our linearly-homomorphic signature scheme, in Theorem 9 we require the scheme $\Sigma^{\prime}$ to be deterministic. It is worth mentioning that alternatively we could also prove context-hiding by requiring $\Sigma^{\prime}$ to be re-randomizable and by giving an explicit Hide algorithm. In a few words, ${ }^{6}$ a signature scheme is re-randomizable if it comes with an additional algorithm $\mathcal{R}$ that on input a message $m$ and a signature $\sigma$ outputs a new signature $\tilde{\sigma}$ which is indistinguishable from a fresh signature on $m$. If $\Sigma^{\prime}$ is re-randomizable, then context-hiding can be achieved by letting Hide work as follows: instead of simply being the identity function, Hide takes a signature $\sigma=\left(\sigma_{\Delta}, Z, R, S\right)$, applies the re-randomization algorithm on $\sigma_{\Delta}$ to obtain another signature $\tilde{\sigma}_{\Delta}$ on the message $(\Delta \mid Z)$, and outputs $\tilde{\sigma}=\left(\tilde{\sigma}_{\Delta}, Z, R, S\right)$. This way the fresh signature $\sigma_{\Delta}$ created by the simulator will be indistinguishable from the one $\tilde{\sigma}_{\Delta}$ in the output of Hide.

\subsection{Proof of correctness}

Theorem 10 If $\Sigma^{\prime}$ is a correct signature scheme, and $\mathrm{H}, \mathrm{H}^{\prime}$ are asymmetric hash functions for bilinear groups, then the scheme HSig satisfies the authentication correctness property.

Proof Let (sk, vk) be a pair of honestly generated keys and let $\sigma \leftarrow \operatorname{Sign}($ sk, $\Delta, \tau, \mathbf{m}$ ) be a honestly generated signature, with $\sigma=\left(\sigma_{A}, Z, R_{\tau}, S_{\tau}\right)$. In order to prove that the

5 We also stress that, by definition, the outputs of these trapdoor algorithms are statistically indistinguishable.

6 A formal definition of re-randomizable signatures can be found in [1]. 
verification algorithm $\operatorname{Ver}\left(\mathrm{vk}, \mathcal{I}_{(\Delta, \tau)}, \mathbf{m}, \sigma\right)$ outputs 1 with all but negligible probability, the first observation to do is that by the correctness of $\Sigma^{\prime}$ the signature $\sigma_{\Delta}$ verifies correctly for $Z$ and $\Delta$. Then, by construction of HSig, we can see that

$$
S_{\tau}=\left(\text { H.PriEval }(\text { sek }, \tau) \cdot R_{\tau} \cdot \prod_{j=1}^{T} \mathrm{H}_{\text {PriEval }}\left(\text { sek }^{\prime}, j\right)^{m_{j}}\right)^{1 / z}
$$

Hence, we have that

$$
\begin{aligned}
& e\left(S_{\tau}, Z\right)=e\left(\left(\text { H.PriEval }(\text { sek }, \tau) \cdot R_{\tau} \cdot \prod_{j=1}^{T} \text { H.PriEval }^{\prime}\left(\text { sek }^{\prime}, j\right)^{m_{j}}\right)^{1 / z}, Z\right) \\
& =e\left(\operatorname{H.PriEval}(\mathrm{sek}, \tau) \cdot R_{\tau} \cdot \prod_{j=1}^{T} \mathrm{H} . \text { PriEval }^{\prime}\left(\mathbf{s e k}^{\prime}, j\right)^{m_{j}}, g_{2}\right) \\
& =e\left(\mathrm{H} . \text { PriEval }(\mathrm{sek}, \tau), g_{2}\right) \cdot e\left(R_{\tau}, g_{2}\right) \cdot e\left(\prod_{j=1}^{T} \mathrm{H} . \text { PriEval }^{\prime}\left(\text { sek }^{\prime}, j\right)^{m_{j}}, g_{2}\right) \\
& =\text { H.PubEval(pek, } \tau) \cdot e\left(R_{\tau}, g_{2}\right) \cdot \prod_{j=1}^{T} \mathrm{H} . \text { PubEval }^{\prime}\left(\text { pek }^{\prime}, j\right)^{m_{j}}
\end{aligned}
$$

where the last equation holds by definition of H.PubEval and H.PubEval'.

Theorem 11 If $\Sigma^{\prime}$ is a correct signature scheme, and $\mathrm{H}, \mathrm{H}^{\prime}$ are asymmetric hash functions for bilinear groups, then the scheme HSig satisfies the evaluation correctness property.

Proof Let (sk, vk) be a pair of honestly generated keys, and let $\left\{\mathbf{m}^{(i)}, \mathcal{P}_{i, \Delta}, \sigma_{i}=\right.$ $\left.\left(\sigma_{\Delta}, Z, R_{i}, S_{i}\right)\right\}_{i=1}^{\ell}$ be messages, labeled programs and signatures such that $\operatorname{Ver}\left(\mathrm{vk}, \mathcal{P}_{i, \Delta}\right.$, $\left.\mathbf{m}^{(i)}, \sigma_{i}\right)=1$, for all $i=1$ to $\ell$. Let $\sigma \leftarrow$ Eval $\left(\mathrm{vk}, f, \boldsymbol{\sigma}=\left(\sigma_{1}, \ldots, \sigma_{\ell}\right)\right)$ be a a signature obtained by running Eval on signatures $\left(\sigma_{1}, \ldots, \sigma_{\ell}\right)$, where $\sigma=\left(\sigma_{\Delta}, Z, R, S\right)$. By construction of Eval, we have $R=\prod_{i=1}^{\ell} R_{i}^{f_{i}}$ and $S=\prod_{i=1}^{\ell} S_{i}^{f_{i}}$. So, if we let $\mathbf{m}=f\left(\mathbf{m}^{(1)}, \ldots, \mathbf{m}^{(\ell)}\right)=\sum_{i=1}^{\ell} f_{i} \cdot \mathbf{m}^{(i)}$, for evaluation correctness we want to prove that the verification algorithm $\operatorname{Ver}\left(\mathrm{vk}, \mathcal{P}_{\Delta}, \mathbf{m}, \sigma\right)$ outputs 1 .

The fact that $\sigma_{\Delta}$ verifies correctly for $Z$ and $\Delta$ is immediate by correctness of $\Sigma^{\prime}$ and by construction of Eval (which simply copies one of these honestly-generated signatures).

Since each $\sigma_{i}$ verifies correctly, for every $i=1, \ldots, \ell$ we have

$$
e\left(S_{i}, Z\right)=\text { H.PubEval }\left(\text { pek, } \tau_{i}\right) \cdot e\left(R_{i}, g_{2}\right) \cdot \prod_{j=1}^{T} \mathrm{H}_{\mathrm{PubEval}}^{\prime}\left(\text { pek }^{\prime}, j\right)^{m_{j}^{(i)}}
$$

Then, by the previous equations and the fact that $\mathrm{H}, \mathrm{H}^{\prime}$ are asymmetric hash functions for bilinear groups, we obtain the desired equation:

$e(S, Z)=e\left(\prod_{i=1}^{\ell} S_{i}^{f_{i}}, Z\right)$ 


$$
\begin{aligned}
& =e\left(\prod_{i=1}^{\ell}\left(\text { H.PriEval }(\text { sek }, \tau)^{f_{i}} \cdot R_{i}^{f_{i}} \cdot \prod_{j=1}^{T} \mathrm{H} \text { PriEval }^{\prime}\left(\text { sek }^{\prime}, j\right)^{f_{i} m_{j}^{(i)}}\right)^{1 / z}, Z\right) \\
& =\left(\prod_{i=1}^{\ell} \mathrm{H} . \mathrm{PubEval}(\text { pek }, \tau)^{f_{i}}\right) \cdot e\left(R, g_{2}\right) \cdot\left(\prod_{j=1}^{T} \mathrm{H} . \mathrm{PubEval}^{\prime}\left(\text { pek }^{\prime}, j\right)^{\sum_{i=1}^{\ell} f_{i} m_{j}^{(i)}}\right) \\
& =\left(\prod_{i=1}^{\ell} \mathrm{H} . \text { PubEval }(\text { pek }, \tau)^{f_{i}}\right) \cdot e\left(R, g_{2}\right) \cdot\left(\prod_{j=1}^{T} \mathrm{H} \mathrm{PubEval}^{\prime}\left(\text { pek }^{\prime}, j\right)^{m_{j}}\right) \text {. }
\end{aligned}
$$

\subsection{Proof of security}

To prove Theorem 8 , we show that for every PPT adversary $\mathcal{A}$ running in the security experiment HomUF-CMA ${ }_{\mathcal{A}, \mathrm{HSig}}$, the probability that the experiment outputs 1 is negligible. We do the proof by describing a series of hybrid games. We write $G_{i}(\mathcal{A})$ to denote the event that a run of Game $i$ with adversary $\mathcal{A}$ returns 1 . Some of the games use some flag values $\operatorname{bad}_{i}$ that are initially set to false. If at the end of a game any of these values is set to true, the game simply outputs 0 . We call $\mathrm{Bad}_{i}$ the event that $\mathrm{bad}_{i}$ is set to true during the run of an experiment. Essentially, whenever an event $\operatorname{Bad}_{i}$ occurs in Game $i$, the game may deviate its outcome.

Finally, we note that in the following proof we directly use the result of Proposition 1 so that we only have to deal with Type- 1 and Type- 2 forgeries, since Type- 3 ones can be converted in Type-2.

Game 0 This game is the security experiment HomUF-CMA $A_{\mathcal{A}, \mathrm{HSig}}$ (where $\mathcal{A}$ only outputs Type- 1 or Type- 2 forgeries).

Game 1 This game is defined as Game 0 apart from the fact that whenever $\mathcal{A}$ returns a forgery $\sigma^{*}=\left(\sigma_{\Delta}^{*}, Z^{*}, R^{*}, S^{*}\right)$ such that $Z^{*}$ was not generated by the challenger in the signing query phase, then Game 1 sets bad $_{1} \leftarrow$ true. As we show in Lemma 3 , any noticeable difference between Game 0 and Game 1 can be reduced to producing a forgery for the regular signature scheme $\Sigma^{\prime}$. Furthermore, it is worth noting that after this change, the game never outputs 1 if the adversary returns a Type- 1 forgery.

Game 2 This game is defined as Game 1 except that the pseudorandomfunction $F$ is replaced by a random function $\mathcal{R}:\{0,1\}^{*} \rightarrow \mathbb{Z}_{p}$. It is easy to see that Game 1 is computationally indistinguishable from Game 2 under the assumption that PRF.KG is pseudorandom.

Game 3 is defined as Game 2 except for the following change. Let $\left(\mathcal{P}_{\Lambda^{*}}^{*}, \sigma^{*}, \mathbf{m}^{*}\right)$ be the forgery returned by the adversary where $\mathcal{P}_{\Lambda^{*}}^{*}=\left(\mathbf{f}^{*}, \mathcal{L}^{*}\right), \sigma^{*}=\left(\sigma_{\Lambda}^{*}, Z^{*}, R^{*}, S^{*}\right)$ and $\Delta^{*}=\Delta_{\mu}$ for some $\mu \in[Q]$ where $Q$ is the number of distinct datasets asked by $\mathcal{A}$ during the game (note that such $\mu$ must exist at this point since the adversary can win only with a Type-2 forgery). The challenger computes $\hat{S}=$ $\prod_{\tau \in \mathcal{L}^{*}}\left(S_{\tau}\right)^{f_{\tau}^{*}}, \hat{R}=\prod_{\tau \in \mathcal{L}^{*}}\left(R_{\tau}\right)^{f_{\tau}^{*}} \hat{\mathbf{m}}=\sum_{\tau \in \mathcal{L}^{*}} f_{\tau}^{*} \cdot \mathbf{m}_{\tau}$ where $\left\{R_{\tau}, S_{\tau}\right\}_{\tau}$ are the signature components generated by the challenger in all the signing queries $\left(\Delta_{\mu}, \tau, \mathbf{m}_{\tau}\right)$. If the forgery verifies correctly, i.e., $\operatorname{Ver}\left(\mathrm{vk}, \mathcal{P}_{\Delta^{*}}^{*}, \mathbf{m}^{*}, \sigma^{*}\right)=1$, and $\mathbf{m}^{*} \neq \hat{\mathbf{m}}$ and $S^{*}=\hat{S}$, then the challenger sets $\operatorname{bad}_{3} \leftarrow$ true. It is easy to see that $\operatorname{Pr}\left[G_{2}(\mathcal{A})\right]-\operatorname{Pr}\left[G_{3}(\mathcal{A})\right] \leq \operatorname{Pr}\left[\operatorname{Bad}_{3}\right]$. In Lemma 5 we show that any adversary for which $\mathrm{Bad}_{3}$ occurs can be reduced to a solver for the 1-DHI problem. 
Game 4 This game proceeds as Game 3 except for the following change: at the beginning, the challenger chooses a random index $\mu \stackrel{\$}{\leftarrow}[Q]$, where $Q=\operatorname{poly}(\lambda)$ is the number of signing queries made by $\mathcal{A}$ during the game. Let $\Delta_{1}, \ldots, \Delta_{Q}$ be all the datasets queried by $\mathcal{A}$. Then if the dataset $\Delta^{*}$ used by $\mathcal{A}$ in the forgery is not $\Delta_{\mu}$, the challenger sets bad $_{4} \leftarrow$ true. As one can check, we have that $\operatorname{Pr}\left[G_{3}(\mathcal{A})\right]=$ $Q \cdot \operatorname{Pr}\left[G_{4}(\mathcal{A})\right]$.

Game 5 proceeds as Game 4 except that at the end the challenger runs the following additional check: if $\operatorname{Ver}\left(\mathrm{vk}, \mathcal{P}_{A^{*}}^{*}, \mathbf{m}^{*}, \sigma^{*}\right)=1$ and $\mathbf{m}^{*} \neq \hat{\mathbf{m}}$ and $S^{*} \neq \hat{S}$ and $R^{*}=\hat{R}$, then the challenger sets bad b $_{5} \leftarrow$ true. It is easy to see that $\operatorname{Pr}\left[G_{4}(\mathcal{A})\right]-\operatorname{Pr}\left[G_{5}(\mathcal{A})\right] \leq$ $\operatorname{Pr}\left[\mathrm{Bad}_{5}\right]$. In Lemma 7 we show that any adversary for which $\mathrm{Bad}_{5}$ occurs can be reduced to a solver for the $2-\mathrm{DHI}$ problem.

Game 6 proceeds as Game 5 with the following modification. At the very beginning, the challenger chooses the value $z_{\mu} \stackrel{\$}{\leftarrow} \mathbb{Z}_{p}$ that will be used to generate the signatures for $\mu$-th dataset $\Delta_{\mu}$. It sets $Z_{\mu}=g_{2}^{z_{\mu}}$. Second, instead of generating the key pek of the hash function $H$ using H.Gen, the challenger runs (td, pek) $\stackrel{\$}{\leftarrow}$ $H$. TrapGen $\left(1^{\lambda}\right.$, bgp, $\left.g_{1}, g_{1}, g_{2}, Z_{\mu}\right)$ where $H$. TrapGen is the algorithm for which $H$ has $(1, \gamma, \epsilon)$-programmable pseudorandomness. Then the challenger uses $t d$ when it needs to compute $\mathrm{H}(\cdot)$ during the experiment.

If $H$ hash $(1, \gamma, \epsilon)$-programmable pseudorandomness we immediately obtain that Game 5 and Game 6 are within statistical distance $\gamma$, i.e., $\mid \operatorname{Pr}\left[G_{5}(\mathcal{A})\right]-$ $\operatorname{Pr}\left[G_{6}(\mathcal{A})\right] \mid \leq \gamma$.

Game 7 This game is the same as Game 6, except that in the signing queries $(\Delta, \tau, \mathbf{m})$ such that $\Delta$ is the $\mu$-th distinct dataset queried by $\mathcal{A}$, the challenger first computes $\mathbf{c}_{\tau} \leftarrow \mathrm{H}$. TrapEval(td, $\tau$ ) and then generates the signature component $R_{\tau}$ by setting $R_{\tau}=g_{1}^{-c_{\tau, 0}}$, instead of choosing $R_{\tau} \stackrel{\$}{\leftarrow} \mathbb{G}_{1}$ randomly as done up to Game 6 .

As we show in Lemma 8 , Game 6 is computationally indistinguishable from Game 7 under the assumption that $\mathrm{H}$ has programmable pseudorandomness. Moreover, note that due to the previous modifications, Game 7 can output 1 only if the adversary outputs a forgery $\left(\mathcal{P}_{\Delta^{*}}^{*}, \sigma^{*}, \mathbf{m}^{*}\right)$ such that $\operatorname{Ver}\left(\mathbf{v k}, \mathcal{P}_{\Delta^{*}}^{*}, \mathbf{m}^{*}, \sigma^{*}\right)=1$ and $\mathbf{m}^{*} \neq \hat{\mathbf{m}}$ and $S^{*} \neq \hat{S}$ and $R^{*} \neq \hat{R}$. We conclude the proof by showing in Lemma 9 that an adversary that wins in Game 7 can be used to solve the FDHI problem (Definition 4).

We proceed with the proof by formally bounding the difference between each consecutive pair of games, and eventually the probability that an adversary wins in the last game. The proof of Theorem 8 is finally obtained by putting together all the bounds.

Lemma 3 For every PPT $\mathcal{A}$ there exists a PPT forger $\mathcal{F}$ such that $\operatorname{Pr}\left[G_{0}(\mathcal{A})\right]-\operatorname{Pr}\left[G_{1}(\mathcal{A})\right] \leq$ $\operatorname{Adv} \underset{\sigma^{\prime}, \mathcal{F}}{\mathrm{UF}-\mathrm{CMA}}(\lambda)$.

Proof The two games differ only if $\mathrm{Bad}_{1}$ occurs in Game 1, i.e., $\left|\operatorname{Pr}\left[G_{0}(\mathcal{A})\right]-\operatorname{Pr}\left[G_{1}(\mathcal{A})\right]\right| \leq$ $\operatorname{Pr}\left[\mathrm{Bad}_{1}\right]$. However, by the construction of HSig, if Bad $\mathrm{B}_{1}$ occurs, it means that the forgery returned by $\mathcal{A}$ includes a valid signature $\sigma_{\Delta^{*}}$ on $\left(\Delta^{*} \mid Z^{*}\right)$ although no signature on $\left(\Delta^{*} \mid \cdot\right)$ was ever returned by the challenger during the experiment. It is straightforward to show that, for any such a PPT $\mathcal{A}$, there exists a PPT forger algorithm $\mathcal{F}$ that breaks the unforgeability of the regular signature scheme $\Sigma^{\prime}$, i.e., $\operatorname{Pr}\left[\mathrm{Bad}_{1}\right] \leq \mathbf{A d v}_{\sigma^{\prime}, \mathcal{F}}^{\mathrm{UF}-\mathrm{CMA}}(\lambda)$.

Lemma 4 For every PPT $\mathcal{A}$ there exists a PPT distinguisher $\mathcal{D}$ such that $\mid \operatorname{Pr}\left[G_{1}(\mathcal{A})\right]-$ $\operatorname{Pr}\left[G_{2}(\mathcal{A})\right] \mid \leq \mathbf{A d v}_{F, \mathcal{D}}^{P R F}(\lambda)$. 
Proof Game 1 and Game 2 differ just for the fact that the PRF $F$ is replaced by a random function $\mathcal{R}$. It is easy to do a reduction to the security of the PRF to show that for any adversary $\mathcal{A}$ such that $\left|\operatorname{Pr}\left[G_{1}(\mathcal{A})\right]-\operatorname{Pr}\left[G_{2}(\mathcal{A})\right]\right| \geq \epsilon$ is non-negligible it is possible to construct a PPT distinguisher $\mathcal{D}$ that archives advantage $\epsilon$ against the pseudorandomness of $F$.

Lemma 5 If $\mathrm{H}$ is simply (1, $\gamma$ )-programmable, and $\mathrm{H}^{\prime}$ is weakly (poly, 1, 2, $\gamma^{\prime}, \delta^{\prime}$ )-degree-2 programmable, then for every PPT A running in Game 3 there exists a PPT simulator $\mathcal{B}$ such that $\operatorname{Pr}\left[\mathrm{Bad}_{3}\right] \leq\left(T / \delta^{\prime}\right) \cdot \mathbf{A d v}_{\mathcal{B}}^{1-D H I}(\lambda)+\gamma+\gamma^{\prime}$.

Proof Assume there exists a PPT adversary $\mathcal{A}$ such that $\operatorname{Pr}\left[\mathrm{Bad}_{3}\right] \geq \epsilon$. Then we show how to build a PPT simulator $\mathcal{B}$ that breaks the 1-DHI assumption with advantage greater than $\left(\delta^{\prime} \epsilon\right) / T-\gamma-\gamma^{\prime}$.

$\mathcal{B}$ takes as input a tuple $\left(g_{1}, g_{2}, g_{1}^{z}, g_{2}^{z}\right)$, and its goal is to compute $g_{1}^{z^{2}}$. Precisely, here we use the fact that this problem is equivalent to the 1-DHI problem in which the adversary has to compute $g_{1}^{1 / z}$. So, $\mathcal{B}$ proceeds as follows.

Setup: $\quad \mathcal{B}$ starts by sampling a random $y \stackrel{\$}{\leftarrow} \mathbb{Z}_{p}$ and runs (td, pek) $\stackrel{\$}{\leftarrow}$ H.TrapGen $\left(1^{\lambda}, \operatorname{bgp}, g_{1}, g_{1}, g_{2}, g_{2}^{y}\right)$. Note that since $\mathcal{B}$ had set $h_{1}=g_{1}$, the polynomials $c_{X}$ generated by $H$. TrapEval $(\mathrm{td}, X)$ will be univariate polynomials $c_{X}(y)$. Next, it chooses a random index $v \stackrel{S}{\leftarrow}[T]$, which represents a guess on the index where the message vector $\mathbf{m}^{*}$ returned by the adversary in the forgery will differ from the "correct" result $\hat{\mathbf{m}}$. Then $\mathcal{B}$ sets $h_{1}=g_{1}^{z}, h_{2}=g_{2}^{z}$ and runs the trapdoor generation (for weakly degree-2 programmability) of the asymmetric hash function $\mathrm{H}^{\prime}$ - $\left(\mathrm{td}^{\prime}\right.$, pek') $\stackrel{\$}{\leftarrow} \mathrm{H}^{\prime}$.TrapGen $\left(1^{\lambda}\right.$, bgp, $\left.g_{1}, h_{1}, g_{2}, h_{2}, v\right)$ - by providing $v$ as the input on which the coefficient $c_{\nu, 2} \neq 0$. Indeed, notice that by giving $h_{1}=g_{1}^{z}, h_{2}=g_{2}^{z}$ to $\mathrm{H}^{\prime}$.TrapGen, the polynomials generated $\mathrm{H}^{\prime}$.TrapEval $\left(\mathrm{td}^{\prime}, X\right)$ will be univariate polynomials $c_{X}(z)$.

Finally, the simulator generates the keys $\left(\mathrm{sk}^{\prime}, \mathrm{vk}^{\prime}\right)$ of the scheme $\Sigma^{\prime}$, sets $\mathrm{vk}=\left(\mathrm{vk}^{\prime}\right.$, pek, pek'), stores sk', td, td', and returns vk to $\mathcal{A}$.

Signing queries: Let $k \leftarrow 1$ be a counter for the number of datasets queried by $\mathcal{A}$. For every new queried dataset $\Delta, \mathcal{B}$ creates a list $T_{\Delta}$ of tuples $(\tau, \mathbf{m}, \sigma)$, which collects all the label/message pairs queried by the adversary on $\Delta$, and the respectively generated signatures. Moreover, whenever the $k$-th new dataset $\Delta_{k}$ is queried, $\mathcal{B}$ samples a random $\xi_{k} \stackrel{\$}{\leftarrow} \mathbb{Z}_{p}$, computes $Z_{k}=\left(g_{2}^{z}\right)^{\xi_{k}}$ and stores $\xi_{k}$. Note that all the values $\left\{Z_{k}\right\}_{k \in[Q]}$ are random in $\mathbb{G}_{2}$ and thus are distributed exactly as in Game 3 .

Given a signing query $(\Delta, \tau, \mathbf{m})$ such that $\Delta=\Delta_{k}$ is the $k$-th dataset, $\mathcal{B}$ proceeds as follows. First, it runs $\mathbf{c}_{\tau} \leftarrow H$. TrapEval $(\mathrm{td}, \tau)$, and $\mathbf{c}_{j}^{\prime} \leftarrow \mathrm{H}^{\prime}$. TrapEval $\left(\mathrm{td}^{\prime}, j\right)$ for all $j=1$ to $T$. If $c_{j, 2}^{\prime}=0$ then $\mathcal{B}$ continues the simulation as follows, otherwise it aborts.

Therefore, $\mathcal{B}$ samples a random $\rho_{\tau} \stackrel{\$}{\leftarrow} \mathbb{Z}_{p}$ and computes

$R_{\tau}=g_{1}^{-c_{\tau}(y)-\sum_{j=1}^{T} c_{j, 0}^{\prime} m_{j}} \cdot\left(g_{1}^{z}\right)^{\rho_{\tau}}, \quad S_{\tau}=\left(g_{1}^{\rho_{\tau}} \cdot g_{1}^{\sum_{j=1}^{T} c_{j, 1}^{\prime} m_{j}} \cdot\left(g_{1}^{z}\right)^{c_{\nu, 2}^{\prime} m_{\nu}}\right)^{\frac{1}{\xi_{k}}}$ 
As one can see, the value $R_{\tau}$ is a uniformly distributed $\mathbb{G}_{1}$ element as in Game 3. Moreover, $S_{\tau}$ is a correctly distributed signature since

$$
\begin{aligned}
& S_{\tau}=\left(g_{1}^{\rho_{\tau}} \cdot g_{1}^{\sum_{j=1}^{T} c_{j, 1}^{\prime} m_{j}} \cdot\left(g_{1}^{z}\right)^{c_{v, 2}^{\prime} m_{\nu}}\right)^{\frac{1}{\xi k}}=\left(g_{1}^{z \rho_{\tau}} \cdot g_{1}^{\sum_{j=1}^{T} z c_{j, 1}^{\prime} m_{j}} \cdot\left(g_{1}^{z^{2}}\right)^{c_{v, 2}^{\prime} m_{\nu}}\right)^{\frac{1}{\xi_{k}}} \\
& =\left(g_{1}^{c_{\tau}(y)} \cdot g_{1}^{-c_{\tau}(y)-\sum_{j=1}^{T} c_{j, 0}^{\prime} m_{j}} \cdot\left(g_{1}^{z}\right)^{\rho_{\tau}} \cdot g_{1}^{\sum_{j=1}^{T} c_{j, 0}^{\prime} m_{j}} \cdot g_{1}^{\sum_{j=1}^{T} z c_{j, 1}^{\prime} m_{j}} \cdot\left(g_{1}^{z^{2}}\right)^{c_{\gamma, 2}^{\prime} m_{v}}\right)^{\frac{1}{z_{k}}} \\
& =\left(\mathrm{H}(\tau) \cdot R_{\tau} \cdot g_{1}^{\sum_{j=1}^{T}\left(c_{j, 0}^{\prime}+c_{j, 1}^{\prime} z+c_{j, 2}^{\prime} z^{2}\right) m_{j}}\right)^{\frac{1}{z_{k}}} \\
& =\left(\mathrm{H}(\tau) \cdot R_{\tau} \cdot \prod_{j=1}^{T} g_{1}^{c_{j}^{\prime}(z) m_{j}}\right)^{\frac{1}{z_{k}}}=\left(\mathrm{H}(\tau) \cdot R_{\tau} \cdot \prod_{j=1}^{T} \mathrm{H}^{\prime}(j)^{m_{j}}\right)^{\frac{1}{z_{k}}}
\end{aligned}
$$

Finally, $\mathcal{B}$ returns to $\mathcal{A}$ the signature $\sigma=\left(\sigma_{\Delta}, Z_{k}, R_{\tau}, S_{\tau}\right)$, where $\sigma_{\Delta} \stackrel{\$}{\leftarrow}$ $\operatorname{Sign}\left(\mathrm{sk}^{\prime}, \Delta \mid Z_{k}\right)$.

Forgery: $\quad$ Let $\left(\mathcal{P}_{\Delta^{*}}^{*}, \sigma^{*}, \mathbf{m}^{*}\right)$ be the forgery returned by the adversary. $\mathcal{B}$ proceeds exactly as the challenger in Game 3 in order to compute $\hat{R}, \hat{S}, \hat{\mathbf{m}}$. If $\operatorname{Bad}_{3}$ occurs, since $\left(\mathcal{P}_{\Delta^{*}}^{*}, \sigma^{*}, \mathbf{m}^{*}\right)$ verifies correctly the following two equations hold

$$
\begin{gathered}
e\left(S^{*}, Z_{\mu}\right)=\Lambda \cdot e\left(R^{*}, g_{2}\right) \cdot \prod_{j=1}^{T} \mathrm{H} . \text { PubEval' }\left(\text { pek }^{\prime}, j\right)^{m_{j}^{*}}, \\
e\left(\hat{S}, Z_{\mu}\right)=\Lambda \cdot e\left(\hat{R}, g_{2}\right) \cdot \prod_{j=1}^{T} \mathrm{H} . P^{\prime} \operatorname{Puvval}^{\prime}\left(\text { pek }^{\prime}, j\right)^{\hat{m}_{j}}
\end{gathered}
$$

where $\Lambda=\prod_{\tau \in \mathcal{L}^{*}}$ H.PubEval(pek, $\left.\tau\right)^{f_{\tau}^{*}}$. If we divide the two equations and consider that, by definition of $\operatorname{Bad}_{3}$, it holds $S^{*}=\hat{S}$, then we obtain

$$
\frac{\hat{R}}{R^{*}}=\prod_{j=1}^{T} H^{\prime}(j)^{m_{j}^{*}-\hat{m}_{j}}
$$

By correctness of the trapdoor algorithms of $\mathrm{H}^{\prime}$ we know that $\mathrm{H}^{\prime}(j)=$ $g_{1}^{c_{j, 0}^{\prime}+c_{j, 1}^{\prime} z+c_{j, 2}^{\prime} z^{2}}$ where $c_{j, 2}^{\prime}=0$ for all $j \neq \nu$ (since $\mathcal{B}$ did not abort so far). If $c_{\nu, 2}^{\prime}=0$ then $\mathcal{B}$ aborts, otherwise it continues as follows. However, notice that by the weak (poly, $1,2, \gamma^{\prime}, \delta^{\prime}$ ) programmability of $\mathrm{H}^{\prime}$ the event that $c_{j, 2}^{\prime}=0$ for all $j \neq v$ and $c_{v, 2}^{\prime} \neq 0$ holds with probability $\delta^{\prime}$. Therefore, $\mathcal{B}$ does not abort with probability $\delta^{\prime}$. Furthermore, since the simulation provided to $\mathcal{A}$ until its forgery's output is distributed statistically close to the real execution of Game 3 (close by a factor $\gamma+\gamma^{\prime}$ due to the use of TrapGen in $\mathrm{H}$ and $\mathrm{H}^{\prime}$ ), $v$ is information-theoretically hidden to $\mathcal{A}$. Hence,

$$
\begin{aligned}
\frac{\hat{R}}{R^{*}} & =g_{1}^{\sum_{j=1}^{T}\left(c_{j, 0}^{\prime}+c_{j, 1}^{\prime} z+c_{j, 2}^{\prime} z^{2}\right)\left(m_{j}^{*}-\hat{m}_{j}\right)} \\
& =g_{1}^{\sum_{j=1}^{T}\left(c_{j, 0}^{\prime}+c_{j, 1}^{\prime} z\right)\left(m_{j}^{*}-\hat{m}_{j}\right)+c_{v, 2}^{\prime} z^{2}\left(m_{v}^{*}-\hat{m}_{\nu}\right)}
\end{aligned}
$$


Since $\mathbf{m}^{*} \neq \hat{\mathbf{m}}$ there must exist an index $v^{\prime} \in[T]$ such that $m_{v^{\prime}}^{*} \neq \hat{m}_{v^{\prime}}$. If $v^{\prime} \neq v$ then $\mathcal{B}$ aborts, otherwise it computes

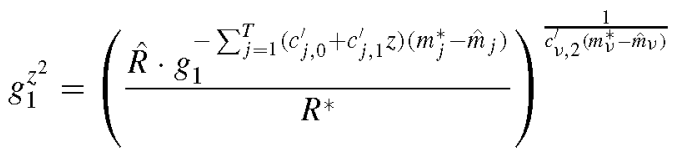

It is easy to see that if $\mathcal{B}$ does not abort, $\mathcal{B}$ is able to compute the solution $g_{1}^{z^{2}}$ of the 1 -DHI problem. The probability that $\mathcal{B}$ does not abort is $\delta^{\prime} \cdot \operatorname{Pr}\left[v^{\prime}=v\right]=\delta^{\prime} / T$ since $v$ is uniformly distributed and completely hidden from the view of $\mathcal{A}$. In conclusion, we have that if $\operatorname{Pr}\left[\mathrm{Bad}_{3}\right] \geq \epsilon$ then $\mathcal{B}$ has advantage at least $\left(\delta^{\prime} \epsilon\right) / T-\gamma-\gamma^{\prime}$.

Lemma $6 \operatorname{Pr}\left[G_{3}(\mathcal{A})\right]=Q \cdot \operatorname{Pr}\left[G_{4}(\mathcal{A})\right]$

Proof First, note that $\operatorname{Pr}\left[G_{4}(\mathcal{A})\right]=\operatorname{Pr}\left[G_{4}(\mathcal{A}) \wedge \operatorname{Bad}_{4}\right]+\operatorname{Pr}\left[G_{4}(\mathcal{A}) \wedge \neg \operatorname{Bad}_{4}\right]=$ $\operatorname{Pr}\left[G_{4}(\mathcal{A}) \mid \neg \mathrm{Bad}_{4}\right] \operatorname{Pr}\left[\neg \mathrm{Bad}_{4}\right]$ since Game 4 outputs 0 whenever Bad 4 occurs. Second, observe that when $\mathrm{Bad}_{4}$ does not occur (i.e., the challenger guesses correctly the query index $\mu$ of the dataset $\Delta^{*}$ ) then the outcome of Game 4 is identical to the one of Game 3, i.e., $\operatorname{Pr}\left[G_{4}(\mathcal{A}) \mid \neg \operatorname{Bad}_{4}\right]=\operatorname{Pr}\left[G_{3}(\mathcal{A})\right]$. Since $\mu$ is chosen uniformly at random and is completely hidden to $\mathcal{A}$ we have that $\operatorname{Pr}\left[\neg \mathrm{Bad}_{4}\right]=1 / Q$, from which the lemma follows.

Lemma 7 If $\mathrm{H}$ is simply $(1, \gamma)$-programmable, and $\mathrm{H}^{\prime}$ is weakly (poly, 1, 2, $\gamma^{\prime}, \delta^{\prime}$ )programmable, then for every PPT $\mathcal{A}$ running in Game 5 there exists a PPT simulator $\mathcal{B}$ such that $\operatorname{Pr}\left[\operatorname{Bad}_{5}\right] \leq\left(T / \delta^{\prime}\right) \cdot \mathbf{A d v}_{\mathcal{B}}^{2-D H I}(\lambda)+\gamma+\gamma^{\prime}$.

Proof Assume there exists a PPT adversary $\mathcal{A}$ such that $\operatorname{Pr}\left[\mathrm{Bad}_{5}\right] \geq \epsilon$. Then we show how to build a PPT simulator $\mathcal{B}$ that breaks the 2-DHI assumption in $\mathbb{G}_{1}$ with advantage greater than $\left(\delta^{\prime} \epsilon\right) / T-\gamma-\gamma^{\prime}$.

$\mathcal{B}$ takes as input a tuple $\left(g_{1}, g_{2}, g_{1}^{z}, g_{2}^{z}, g_{1}^{z^{2}}, g_{2}^{z^{2}}\right)$, and its goal is to compute $g_{1}^{1 / z}$. To do so $\mathcal{B}$ proceeds as follows.

Setup: $\quad \mathcal{B}$ proceeds as the challenger in Game 5 by choosing a random index $\mu \stackrel{\$}{\leftarrow}[Q]$. Second, $\mathcal{B}$ picks a random $y \stackrel{\$}{\leftarrow} \mathbb{Z}_{p}$ and runs (td, pek) $\stackrel{\$}{\leftarrow}$ H. TrapGen $\left(1^{\lambda}, \operatorname{bgp}, g_{1}, g_{1}, g_{2}, g_{2}^{y}\right)$. Note that since $\mathcal{B}$ had set $h_{1}=g_{1}$, the polynomials $c_{X}$ generated by $\mathrm{H}$. TrapEval $(\mathrm{td}, X)$ will be univariate, degree-1, polynomials $c_{X}(y)$. Next, it chooses a random index $\nu \stackrel{\$}{\leftarrow}[T]$, which represents a guess on the index where the message vector $\mathbf{m}^{*}$ returned by the adversary in the forgery will differ from the "correct" result $\hat{\mathbf{m}}$. It runs the trapdoor generation (for weak (poly, 1, 2)programmability) of the asymmetric hash function $\mathrm{H}^{\prime}-\left(\mathrm{td}^{\prime}, \mathrm{pek}^{\prime}\right) \stackrel{\$}{\leftarrow}$ $H^{\prime}$.TrapGen( $\left(1^{\lambda}\right.$, bgp $\left., g_{1}, g_{1}^{z}, g_{2}, g_{2}^{z}, v\right)$ - providing $v$ as the input on which the coefficient $c_{\nu, 0} \neq 0$. Notice that by giving $h_{1}=g_{1}^{z}, h_{2}=g_{2}^{z}$ to $\mathrm{H}^{\prime}$. TrapGen, the polynomials generated $\mathrm{H}^{\prime}$.TrapEval $\left(\mathrm{td}^{\prime}, X\right)$ will be univariate polynomials $c_{X}(z)=c_{X, 0}+c_{X, 1} z+c_{X, 2} z^{2}$.

Finally, it generates the keys $\left(\mathrm{sk}^{\prime}, \mathrm{vk}^{\prime}\right)$ of the scheme $\Sigma^{\prime}$, sets $\mathrm{vk}=$ $\left(\mathrm{vk}^{\prime}\right.$, pek, pek'), stores sk', td, $\mathrm{td}^{\prime}$, and returns vk to $\mathcal{A}$.

Signing queries: $\quad$ Let $k \leftarrow 1$ be a counter for the number of datasets queried by $\mathcal{A}$. For every new queried dataset $\Delta, \mathcal{B}$ creates a list $T_{\Delta}$ of tuples $(\tau, \mathbf{m}, \sigma)$, which collects all the label/message pairs queried by the adversary on $\Delta$ and the respectively generated signatures. Moreover, whenever the $k$-th new 
dataset $\Delta_{k}$ is queried, $\mathcal{B}$ does the following: if $k=\mu$ it samples a random $\xi \stackrel{\$}{\leftarrow} \mathbb{Z}_{p}$, computes $Z_{\mu}=\left(g_{2}^{z}\right)^{\xi}$ and stores $\xi$; if $k \neq \mu, \mathcal{B}$ samples directly a random $z_{k} \stackrel{\$}{\leftarrow} \mathbb{Z}_{p}$, computes $Z_{k}=g_{2}^{z_{k}}$ and stores $z_{k}$. Note that all the values $\left\{Z_{k}\right\}_{k \in[Q]}$ are random in $\mathbb{G}_{2}$ and thus are distributed exactly as in Game 5.

Given a signing query $(\Delta, \tau, \mathbf{m})$ such that $\Delta=\Delta_{k}$ is the $k$-th dataset, $\mathcal{B}$ first computes $\sigma_{\Delta_{k}} \leftarrow \operatorname{Sign}\left(\mathrm{sk}^{\prime}, \Delta_{k}, Z_{k}\right.$ ), and then proceeds as follows.

- If $k \neq \mu, \mathcal{B}$ runs $\mathbf{c}_{\tau} \leftarrow \mathrm{H}$. TrapEval $(\mathrm{td}, \tau)$, and $\mathbf{c}_{j}^{\prime} \leftarrow \mathrm{H}^{\prime}$. TrapEval $\left(\mathrm{td}^{\prime}, j\right)$ for all $j=1$ to $T$. It samples $R_{\tau} \stackrel{\$}{\leftarrow} \mathbb{G}_{1}$, and computes

$$
S_{\tau}=\left(g_{1}^{c_{\tau}(y)} \cdot R_{\tau} \cdot \prod_{j=1}^{T} g_{1}^{c_{j}^{\prime}(z) m_{j}}\right)^{\frac{1}{z_{k}}}
$$

In particular, note that every $g_{1}^{c_{j}^{\prime}(z)}$ can be computed by $\mathcal{B}$ using the values $g_{1}^{z}, g_{1}^{z^{2}}$

- If $k=\mu, \mathcal{B}$ runs $\mathbf{c}_{\tau} \leftarrow \mathrm{H}$. TrapEval (td, $\left.\tau\right)$, and $\mathbf{c}_{j}^{\prime} \leftarrow \mathrm{H}^{\prime}$. TrapEval $\left(\mathrm{td}^{\prime}, j\right)$ for all $j=1$ to $T$. If $c_{j, 0}^{\prime}=0$ for all $j \neq v$, then $\mathcal{B}$ continues the simulation as follows, otherwise it aborts.

Therefore, $\mathcal{B}$ samples a random $\rho_{\tau} \stackrel{\$}{\leftarrow} \mathbb{Z}_{p}$ and computes

$$
R_{\tau}=g_{1}^{-c_{\tau}(y)-c_{\nu, 0} m_{v}} \cdot\left(g_{1}^{z}\right)^{\rho_{\tau}}, \quad S_{\tau}=\left(g_{1}^{\rho_{\tau}} \cdot g_{1}^{\sum_{j=1}^{T}\left(c_{j, 1}^{\prime}+c_{j, 2}^{\prime} z\right) m_{j}}\right)^{\frac{1}{\xi}}
$$

As one can see, the value $R_{\tau}$ is a uniformly distributed $\mathbb{G}_{1}$ element as in Game 5. Moreover, $S_{\tau}$ is a correctly distributed signature since

$$
\begin{aligned}
S_{\tau} & =\left(g_{1}^{\rho_{\tau}} \cdot g_{1}^{\sum_{j=1}^{T}\left(c_{j, 1}^{\prime}+c_{j, 2}^{\prime} z\right) m_{j}}\right)^{\frac{1}{\xi}}=\left(g_{1}^{z \rho_{\tau}} \cdot g_{1}^{\sum_{j=1}^{T}\left(c_{j, 1}^{\prime} z+c_{j, 2}^{\prime} z^{2}\right) m_{j}}\right)^{\frac{1}{z \xi}} \\
& =\left(g_{1}^{c_{\tau}(y)} \cdot g_{1}^{-c_{\tau}(y)-c_{\nu, 0}^{\prime} m_{v}} \cdot g_{1}^{z \rho_{\tau}} \cdot g_{1}^{\sum_{j=1}^{T}\left(c_{j, 0}^{\prime}+z c_{j, 1}^{\prime}+c_{j, 2}^{\prime} z^{2}\right) m_{j}}\right)^{\frac{1}{z \xi}} \\
& =\left(\mathrm{H}(\tau) \cdot R_{\tau} \cdot g_{1}^{\sum_{j=1}^{T}\left(c_{j, 0}^{\prime}+z c_{j, 1}^{\prime}+c_{j, 2}^{\prime} z^{2}\right) m_{j}}\right)^{\frac{1}{z \xi}} \\
& =\left(\mathrm{H}(\tau) \cdot R_{\tau} \cdot \prod_{j=1}^{T} \mathrm{H}^{\prime}(j)^{m_{j}}\right)^{\frac{1}{z \mu}}
\end{aligned}
$$

Finally, $\mathcal{B}$ returns to $\mathcal{A}$ the signature $\sigma=\left(\sigma_{\Lambda_{k}}, Z_{k}, R_{\tau}, S_{\tau}\right)$.

Forgery: Let $\left(\mathcal{P}_{\Lambda^{*}}^{*}, \sigma^{*}, \mathbf{m}^{*}\right)$ be the forgery returned by the adversary. $\mathcal{B}$ proceeds exactly as the challenger in Game 5 in order to compute $\hat{R}, \hat{S}, \hat{\mathbf{m}}$. If $\operatorname{Bad}_{5}$ occurs, since $\left(\mathcal{P}_{\Delta^{*}}^{*}, \sigma^{*}, \mathbf{m}^{*}\right)$ verifies correctly the following two equations hold

$$
e\left(S^{*}, Z_{\mu}\right)=\Lambda \cdot e\left(R^{*}, g_{2}\right) \cdot \prod_{j=1}^{T} \text { H.PubEval' }\left(\text { pek }^{\prime}, j\right)^{m_{j}^{*}}
$$




$$
e\left(\hat{S}, Z_{\mu}\right)=\Lambda \cdot e\left(\hat{R}, g_{2}\right) \cdot \prod_{j=1}^{T} \text { H.PubEval }\left(\text { pek }^{\prime}, j\right)^{\hat{m}_{j}}
$$

where $\Lambda=\prod_{\tau \in \mathcal{L}^{*}} \mathrm{H}$.PubEval(pek, $\left.\tau\right)^{f_{\tau}^{*}}$. If we divide the two equations and consider that by definition of $\operatorname{Bad}_{3}, S^{*} \neq \hat{S}$ but $R^{*}=\hat{R}$ we obtain

$$
\frac{S^{*}}{\hat{S}}=\left(\prod_{j=1}^{T} \mathrm{H}^{\prime}(j)^{m_{j}^{*}-\hat{m}_{j}}\right)^{\frac{1}{2 \xi}}
$$

By using the $\mathrm{H}^{\prime}$.TrapEval algorithm we know that $\mathrm{H}^{\prime}(j)=g_{1}^{c_{j, 0}^{\prime}+z c_{j, 1}^{\prime}+c_{j, 2}^{\prime} z^{2}}$ where $c_{j, 0}^{\prime}=0$ for all $j \neq v$ (since $\mathcal{B}$ did not abort so far). If $c_{\nu, 0}^{\prime}=0$, then $\mathcal{B}$ aborts, otherwise it continues as follows. Nevertheless, notice that by weak (poly, 1,2)programmability of $\mathrm{H}^{\prime}, c_{j, 0}^{\prime}=0$ for all $j \neq \nu$, whereas $c_{v, 0}^{\prime} \neq 0$ holds with probability $\delta^{\prime}$, which means that with probability at least $\delta^{\prime} \mathcal{B}$ does not abort. Hence,

$$
\begin{aligned}
\frac{S^{*}}{\hat{S}} & =\left(g_{1}^{1 /(z \xi)}\right)^{\sum_{j=1}^{T}\left(c_{j, 0}^{\prime}+z c_{j, 1}^{\prime}+c_{j, 2}^{\prime} z^{2}\right)\left(m_{j}^{*}-\hat{m}_{j}\right)} \\
& =\left(g_{1}^{1 /(z \xi)}\right)^{c_{v, 0}^{\prime}\left(m_{\nu}^{*}-\hat{m}_{v}\right)} g_{1}^{\sum_{j=1}^{T}\left(c_{j, 1}^{\prime}+c_{j, 2}^{\prime} z\right)\left(m_{j}^{*}-\hat{m}_{j}\right) / \xi}
\end{aligned}
$$

Since $\mathbf{m}^{*} \neq \hat{\mathbf{m}}$ there must exist an index $v^{\prime} \in[T]$ such that $m_{v^{\prime}}^{*} \neq \hat{m}_{v^{\prime}}$. If $v^{\prime} \neq v$ then $\mathcal{B}$ aborts, otherwise it computes

$$
g_{1}^{1 / z}=\left(\frac{S^{*} \cdot g_{1}^{-\sum_{j=1}^{T}\left(c_{j, 1}^{\prime}+c_{j, 2}^{\prime} z\right)\left(m_{j}^{*}-\hat{m}_{j}\right) / \xi}}{\hat{S}}\right)^{\frac{\xi}{c_{\nu, 0}^{\prime}\left(m_{\nu}^{*}-\hat{m}_{\nu}\right)}}
$$

Note that the simulation of Game 5 provided by $\mathcal{B}$ to $\mathcal{A}$ is statistically close (by a factor $\gamma+\gamma^{\prime}$ due to the use of TrapGen in $\mathrm{H}_{\text {and }} \mathrm{H}^{\prime}$ ) to the real execution of Game 5. Then, it is easy to see that if $\mathcal{B}$ does not abort, it is able to compute the solution of the 2-DHI problem $g_{1}^{1 / z}$. The probability that $\mathcal{B}$ does not abort is $\delta^{\prime} \cdot \operatorname{Pr}\left[v^{\prime}=v\right]=\delta^{\prime} / T$ since $v$ is uniformly distributed and completely hidden from the view of $\mathcal{A}$. In conclusion, we have that if $\operatorname{Pr}\left[\operatorname{Bad}_{5}\right] \geq \epsilon$ then $\mathcal{B}$ has advantage at least $\left(\delta^{\prime} \epsilon\right) / T-\gamma-\gamma^{\prime}$.

Lemma 8 If the asymmetric hash function $\mathrm{H}$ has $(1, \gamma, \epsilon)$-programmable pseudorandomness then $\left|\operatorname{Pr}\left[G_{6}(\mathcal{A})\right]-\operatorname{Pr}\left[G_{7}(\mathcal{A})\right]\right| \leq \epsilon$.

Proof We do the proof by contradiction. Assume there exists a PPT adversary $\mathcal{A}$ such that $\left|\operatorname{Pr}\left[G_{1}\right]-\operatorname{Pr}\left[G_{2}\right]\right| \geq \epsilon$. Then we show how to build a PPT simulator $\mathcal{B}$ that breaks the programmable pseudorandomness of $\mathrm{H}$ with advantage $\epsilon$. We build such a simulator $\mathcal{B}$ as follows:

Setup:

$\mathcal{B}$ first receives the bilinear group parameters bgp, which includes the two generators $g_{1}, g_{2} . \mathcal{B}$ proceeds as the challenger in Game 6 by choosing a random index $\mu \stackrel{\$}{\leftarrow}[Q]$ and a random $z_{\mu} \stackrel{\$}{\leftarrow} \mathbb{Z}_{p}$. It also prepares $Z_{\mu}=g_{2}^{z_{\mu}}$. Then it sets $h_{1}=g_{1} \in \mathbb{G}_{1}, h_{2}=Z_{\mu}$ and returns $\left(h_{1}, h_{2}\right)$ to its challenger. It receives back a public key pek for $\mathrm{H}$, and also gets access to an oracle that on input $\tau$ outputs $\mathrm{H}(\tau)$ and either $g_{1}^{c_{\tau, 0}}$ or $g_{1}^{r_{\tau}}$. 
$\mathcal{B}$ then queries its oracle on all inputs $\tau \in[N]$ and stores all the answers $\left\{Y_{\tau}, C_{\tau}\right\}_{\tau \in[N]}$. Moreover, $\mathcal{B}$ chooses in advance the values $z_{k} \stackrel{\$}{\leftarrow} \mathbb{Z}_{p}, \forall k \in[Q] \backslash\{\mu\}$, and stores $\left\{z_{k}, Z_{k}=g_{2}^{z_{k}}\right\}$. Next, it generates the keys $\left(\mathrm{sk}^{\prime}, \mathrm{vk}^{\prime}\right.$ ) of the scheme $\Sigma^{\prime}$, the keys (sek', pek') of the asymmetric hash $\mathrm{H}^{\prime}$, it sets $v \mathbf{k}=\left(\mathrm{vk}^{\prime}\right.$, pek, pek'), stores $\mathrm{sk}^{\prime}$, sek', and returns vk to $\mathcal{A}$.

Signing queries: $\quad$ Let $k \leftarrow 1$ be a counter for the number of datasets queried by $\mathcal{A}$. For every new queried dataset $\Delta, \mathcal{B}$ creates a list $T_{\Delta}$ of tuples $(\tau, \mathbf{m}, \sigma)$, which collects all the label/message pairs queried by the adversary on $\Delta$ and the respectively generated signatures.

On the $k$-th query $(\Delta, \tau, \mathbf{m}), \mathcal{B}$ proceeds as follows:

- If $k \neq \mu, \mathcal{B}$ first generates the signature $\sigma_{\Delta}$ on $\left(\Delta_{k}, Z_{k}\right)$ using the secret key $s k^{\prime}$. Next, it chooses a random value $R_{\tau} \stackrel{\$}{\leftarrow} \mathbb{G}_{1}$ and computes $S_{\tau}=\left(Y_{\tau} \cdot R_{\tau} \prod_{j=1}^{T} \text { H.PriEval' }\left(\text { sek }^{\prime}, j\right)^{m_{j}}\right)^{1 / z_{k}}$.

- If $k=\mu, \mathcal{B}$ works exactly as above except that it sets $R_{\tau}=C_{\tau}^{-1}$.

Finally, $\mathcal{B}$ returns to $\mathcal{A}$ the signature $\sigma=\left(\sigma_{\Delta}, Z_{k}, R_{\tau}, S_{\tau}\right)$, where $\sigma_{\Delta} \stackrel{\$}{\leftarrow} \operatorname{Sign}\left(\mathrm{sk}^{\prime}, \Delta \mid Z_{k}\right)$.

Forgery: Let $\left(\mathcal{P}_{\Delta^{*}}^{*}, \sigma^{*}, \mathbf{m}^{*}\right)$ be the forgery returned by the adversary. $\mathcal{B}$ proceeds exactly as the challenger in Game 7 in order to determine the outcome of the experiment, and outputs 0 or 1 accordingly.

It is easy to see that if $\mathcal{B}$ receives from its oracle values $C_{\tau}$ with the pseudorandom distribution, then $\mathcal{B}$ is perfectly simulating Game 7 to $\mathcal{A}$. Otherwise, if the values $C_{\tau}$ are random then $\mathcal{B}$ is simulating Game 6. Therefore,

$$
\left|\operatorname{Pr}\left[\operatorname{Exp}_{\mathcal{B}, H}^{P R H-0}=1\right]-\operatorname{Pr}\left[\operatorname{Exp}_{\mathcal{B}, H}^{P R H-1}=1\right]\right|=\left|\operatorname{Pr}\left[G_{7}(\mathcal{A})\right]-\operatorname{Pr}\left[G_{6}(\mathcal{A})\right]\right|=\epsilon
$$

To conclude the proof, we are left with showing that any PPT adversary has negligible probability of winning in Game 7. We show this in the following lemma where we prove that this holds under the Flexible Diffie-Hellman Inversion Assumption (FDHI) given in Definition 4.

Lemma 9 If $\mathrm{H}$ has $(1, \gamma, \epsilon)$-programmable pseudorandomness and $\mathrm{H}^{\prime}$ is (poly, $\left.0,1, \gamma^{\prime}, \delta^{\prime}\right)$ programmable, then for any PPT $\mathcal{A}$ running in Game 7 there is a PPT $\mathcal{B}$ against the FDHI assumption such that $\operatorname{Pr}\left[G_{7}(\mathcal{A})\right]=\mathbf{A d v}_{\mathcal{B}}^{F H I}(\lambda) / \delta^{\prime}+\gamma+\gamma^{\prime}$.

Proof Assume that $\mathcal{A}$ is a PPT adversary such that $\operatorname{Pr}\left[G_{7}(\mathcal{A})\right]=\epsilon$. Then we show how to build a PPT simulator $\mathcal{B}$ which uses $\mathcal{A}$ to solve the FDHI problem with advantage $\epsilon$. $\mathcal{B}$ receives an FDHI instance $\left(g_{1}, g_{2}, g_{2}^{z}, g_{2}^{v}, g_{1}^{\frac{z}{v}}, g_{1}^{r}, g_{1}^{\frac{r}{v}}\right)$ and works as follows.

Setup: $\quad \mathcal{B}$ proceeds as the challenger in Game 6 by choosing a random index $\mu \stackrel{\$}{\leftarrow}[Q]$. Next, it runs the trapdoor generation algorithm for the programmable pseudorandomness of $\mathrm{H}$, (td, pek) $\stackrel{\$}{\leftarrow}$ H. TrapGen $\left(1^{\lambda}\right.$, bgp, $\left.g_{1}, g_{1}, g_{2}, g_{2}^{z}\right)$, and the trapdoor generation algorithm for the (poly, 0, 1)-programmability of $\mathrm{H}^{\prime}$, (td' $\mathrm{td}^{\prime}$ pek $) \stackrel{\$}{\leftarrow}$ $H^{\prime}$.TrapGen $\left(1^{\lambda}\right.$, bgp, $\left.g_{1}, g_{1}, g_{2}, g_{2}^{z}\right)$.

Finally, it generates the keys $\left(\mathbf{s k}^{\prime}, \mathrm{vk}^{\prime}\right)$ of the scheme $\Sigma^{\prime}$, sets $\mathrm{vk}=\left(\mathrm{vk}^{\prime}\right.$, pek, pek' $)$, stores sk', td, $\mathrm{td}^{\prime}$, and returns vk to $\mathcal{A}$. 
Let $k \leftarrow 1$ be a counter for the number of datasets queried by $\mathcal{A}$. For every new queried dataset $\Delta, \mathcal{B}$ creates a list $T_{\Delta}$ of tuples $(\tau, \mathbf{m}, \sigma)$, which collects all the label/message pairs queried by the adversary on $\Delta$ and the respectively generated signatures.

Moreover whenever the $k$-th new dataset $\Delta_{k}$ is queried, $\mathcal{B}$ does the following: if $k=\mu$ it samples a random $\xi_{\mu} \stackrel{\$}{\leftarrow} \mathbb{Z}_{p}$, computes $Z_{\mu}=$ $\left(g_{2}^{z}\right)^{\xi_{\mu}}$ and stores $Z_{\mu}, \xi_{\mu}$; if $k \neq \mu, \mathcal{B}$ samples a random $\xi_{k} \stackrel{\$}{\leftarrow} \mathbb{Z}_{p}$, and computes $Z_{k}=\left(g_{2}^{v}\right)^{\xi_{k}}$ and stores $Z_{k}, \xi_{k}$. Note that all the values $\left\{Z_{k}\right\}_{k \in[Q]}$ are random in $\mathbb{G}_{2}$ and thus are distributed exactly as in Game 7.

Given a signing query $(\Delta, \tau, \mathbf{m})$ such that $\Delta=\Delta_{k}$ is the $k$-th dataset, $\mathcal{B}$ first computes $\sigma_{\Delta_{k}} \leftarrow \operatorname{Sign}\left(\mathbf{s k}^{\prime}, \Delta_{k}, Z_{k}\right)$, and then proceeds as follows.

- If $k \neq \mu: \mathcal{B}$ runs $\mathbf{c}_{\tau} \leftarrow \mathrm{H}$.TrapEval (td, $\left.\tau\right)$, and $\mathbf{c}_{j}^{\prime} \leftarrow \mathrm{H}^{\prime}$. TrapEval $\left(\operatorname{td}^{\prime}, j\right)$ for all $j=1$ to $T$. Notice that by the $(1, \gamma, \epsilon)$ programmability of $\mathrm{H}$ we have that $\mathbf{c}_{\tau}$ is a degree-1 polynomial in $z: c_{\tau}(z)=c_{\tau, 0}+c_{\tau, 1} z$. Similarly, the polynomials $\mathbf{c}_{j}^{\prime}$ generated by $\mathrm{H}^{\prime}$.TrapEval are also of degree 1 in the sole variable $z$; If $c_{j, 0}^{\prime}=0$, then $\mathcal{B}$ continues the simulation as follows, otherwise it aborts. Notice that, by (poly, 0, 1)-programmability of $\mathrm{H}^{\prime}$, the event that $\forall j \in[T]: c_{j, 0}^{\prime}=0$, i.e., $c_{j}^{\prime}(z)=c_{j, 1}^{\prime} z$ holds with probability at least $\delta^{\prime}$. Therefore, $\mathcal{B}$ does not abort with probability at least $\delta^{\prime}$.

Next, it samples $\rho_{\tau} \stackrel{\$}{\leftarrow} \mathbb{Z}_{p}$, computes

$$
R_{\tau}=g_{1}^{-c_{\tau, 0}} \cdot\left(g_{1}^{r}\right)^{\rho_{\tau}}, \quad S_{\tau}=\left(\left(g_{1}^{\frac{z}{v}}\right)^{c_{\tau, 1}} \cdot\left(g_{1}^{\frac{r}{v}}\right)^{\rho_{\tau}} \cdot\left(g_{1}^{\frac{z}{v}}\right)^{\sum_{j=1}^{T} c_{j, 1}^{\prime} m_{j}}\right)^{\frac{1}{\xi_{k}}}
$$

and returns $\sigma=\left(Z_{k}, \sigma_{\Delta_{k}}, R_{\tau}, S_{\tau}\right)$ to $\mathcal{A}$.

Note that the signature is correctly distributed as in Game 7, since $R_{\tau}$ is a uniformly distributed $\mathbb{G}_{1}$ element, and

$$
\begin{aligned}
S_{\tau} & =\left(\left(g_{1}^{\frac{z}{v}}\right)^{c_{\tau, 1}} \cdot\left(g_{1}^{\frac{r}{v}}\right)^{\rho_{\tau}} \cdot\left(g_{1}^{\frac{z}{v}}\right)^{\sum_{j=1}^{T} c_{j, 1}^{\prime} m_{j}}\right)^{\frac{1}{\xi_{k}}} \\
& =\left(\left(g_{1}^{z}\right)^{c_{\tau, 1}} \cdot\left(g_{1}^{r}\right)^{\rho_{\tau}} \cdot\left(g_{1}^{z}\right)^{\sum_{j=1}^{T} c_{j, 1}^{\prime} m_{j}}\right)^{\frac{1}{v \xi_{k}}} \\
& =\left(g_{1}^{c_{\tau}, 0} \cdot\left(g_{1}^{z}\right)^{c_{\tau, 1}} \cdot g_{1}^{-c_{\tau, 0}} \cdot\left(g_{1}^{r}\right)^{\rho_{\tau}} \cdot g_{1}^{\sum_{j=1}^{T}\left(c_{j, 1}^{\prime} z\right) m_{j}}\right)^{\frac{1}{z_{k}}} \\
& =\left(\mathrm{H}(\tau) \cdot R_{\tau} \cdot g_{1}^{\sum_{j=1}^{T} c_{j}^{\prime}(z) m_{j}}\right)^{\frac{1}{z_{k}}} \\
& =\left(\mathrm{H}(\tau) \cdot R_{\tau} \cdot \prod_{j=1}^{T} g_{1}^{c_{j}^{\prime}(z) m_{j}}\right)^{\frac{1}{z_{k}}} \\
& =\left(\mathrm{H}(\tau) \cdot R_{\tau} \cdot \prod_{j=1}^{T} \mathrm{H}^{\prime}(j)^{m_{j}}\right)^{\frac{1}{z_{k}}}
\end{aligned}
$$


- If $k=\mu: \mathcal{B}$ runs $\mathbf{c}_{\tau} \leftarrow \mathrm{H}$.TrapEval(td, $\left.\tau\right)$, and $\mathbf{c}_{j}^{\prime} \leftarrow \mathrm{H}^{\prime}$. TrapEval (td', $j$ ) for all $j=1$ to $T$. It sets $R_{\tau}=g_{1}^{-c_{\tau, 0}}$ and computes

$$
S_{\tau}=\left(g_{1}^{c_{\tau, 1}} \cdot g_{1}^{\sum_{j=1}^{T} c_{j, 1}^{\prime} m_{j}}\right)^{\frac{1}{\xi \mu}}
$$

and returns $\sigma=\left(Z_{\mu}, \sigma_{\Delta_{\mu}}, R_{\tau}, S_{\tau}\right)$ to $\mathcal{A}$.

As one can check, such signature is distributed as a signature in Game 7: $R_{\tau}=g_{1}^{-c_{\tau, 0}}$ as in the definition of Game 7 (for the $\mu$-th dataset) while for $S_{\tau}$ we have

$$
\begin{aligned}
S_{\tau} & =\left(g_{1}^{c_{\tau, 1}} \cdot g_{1}^{\sum_{j=1}^{T} c_{j, 1}^{\prime} m_{j}}\right)^{\frac{1}{\xi \mu}}=\left(g_{1}^{z c_{\tau, 1}} \cdot g_{1}^{z \sum_{j=1}^{T} c_{j, 1}^{\prime} m_{j}}\right)^{\frac{1}{z \xi \mu}} \\
& =\left(g_{1}^{c_{\tau, 0}} \cdot g_{1}^{z c_{\tau, 1}} \cdot g_{1}^{-c_{\tau, 0}} \cdot g_{1}^{\sum_{j=1}^{T}\left(c_{j, 1}^{\prime} z\right) m_{j}}\right)^{\frac{1}{z \mu}} \\
& =\left(\mathrm{H}(\tau) \cdot R_{\tau} \cdot g_{1}^{\sum_{j=1}^{T} c_{j}^{\prime}(z) m_{j}}\right)^{\frac{1}{z \mu}} \\
& =\left(\mathrm{H}(\tau) \cdot R_{\tau} \cdot \prod_{j=1}^{T} g_{1}^{c_{j}^{\prime}(z) m_{j}}\right)^{\frac{1}{z \mu}} \\
& =\left(\mathrm{H}(\tau) \cdot R_{\tau} \cdot \prod_{j=1}^{T} \mathrm{H}^{\prime}(j)^{m_{j}}\right)^{\frac{1}{z \mu \nu}}
\end{aligned}
$$

Forgery: Let $\left(\mathcal{P}_{\Lambda^{*}}^{*}, \sigma^{*}, \mathbf{m}^{*}\right)$ be the forgery returned by the adversary. $\mathcal{B}$ proceeds exactly as the challenger in Game 7 in order to compute $\hat{R}, \hat{S}, \hat{\mathbf{m}}$.

By definition, if Game 7 outputs 1 , since $\left(\mathcal{P}_{\Lambda^{*}}^{*}, \sigma^{*}, \mathbf{m}^{*}\right)$ verifies correctly, the following two equations hold

$$
\begin{gathered}
e\left(S^{*}, Z_{\mu}\right)=\Lambda \cdot e\left(R^{*}, g_{2}\right) \cdot \prod_{j=1}^{T} \mathrm{H} . \mathrm{PubEval}^{\prime}\left(\text { pek }^{\prime}, j\right)^{m_{j}^{*}}, \\
e\left(\hat{S}, Z_{\mu}\right)=\Lambda \cdot e\left(\hat{R}, g_{2}\right) \cdot \prod_{j=1}^{T} \mathrm{H} . \mathrm{PubEval}^{\prime}\left(\text { pek }^{\prime}, j\right)^{\hat{m}_{j}}
\end{gathered}
$$

where $\Lambda=\prod_{\tau \in \mathcal{L}^{*}}$ H.PubEval(pek, $\left.\tau\right)^{f_{\tau}^{*}}$. If we divide the two equations and consider that by definition of Game 7, it must be $S^{*} \neq \hat{S}$ and $R^{*} \neq \hat{R}$, then we obtain:

$$
\begin{aligned}
\frac{S^{*}}{\hat{S}} & =\left(\frac{R^{*}}{\hat{R}} \cdot \prod_{j=1}^{T} H^{\prime}(j)^{m_{j}^{*}-\hat{m}_{j}}\right)^{\frac{1}{z \xi \mu}}=\left(\frac{R^{*}}{\hat{R}} \cdot \prod_{j=1}^{T} g_{1}^{c_{j, 1}^{\prime} z\left(m_{j}^{*}-\hat{m}_{j}\right)}\right)^{\frac{1}{z \xi \mu}} \\
& =\left(\frac{R^{*}}{\hat{R}}\right)^{\frac{1}{z \xi \mu}} \cdot\left(\prod_{j=1}^{T} g_{1}^{c_{j, 1}^{\prime}\left(m_{j}^{*}-\hat{m}_{j}\right)}\right)^{\frac{1}{\xi \mu}}
\end{aligned}
$$


Therefore $\mathcal{B}$ can compute

$$
W=\frac{R^{*}}{\hat{R}}, \quad W^{\prime}=\left(\frac{S^{*}}{\hat{S}}\right)^{\xi_{\mu}} \cdot g_{1}^{\sum_{j=1}^{T} c_{j, 1}^{\prime}\left(\hat{m}_{j}-m_{j}^{*}\right)}
$$

and returns $\left(W, W^{\prime}\right)$ as a solution for the FDHI assumption.

To see that $\left(W, W^{\prime}\right)$ is a solution for the FDHI assumption, i.e., $W^{\prime}=W^{1 / z}$, observe that by Eq. (2) it holds

$$
\begin{aligned}
\left(W^{\prime}\right)^{z} & =\left(\frac{S^{*}}{\hat{S}}\right)^{z \xi_{\mu}} \cdot g_{1}^{\sum_{j=1}^{T} z c_{j, 1}^{\prime}\left(\hat{m}_{j}-m_{j}^{*}\right)} \\
& =\left[\left(\frac{R^{*}}{\hat{R}}\right)^{\frac{1}{z \xi_{\mu}}} \cdot\left(\prod_{j=1}^{T} g_{1}^{c_{j, 1}^{\prime}\left(m_{j}^{*}-\hat{m}_{j}\right)}\right)^{\frac{1}{\xi \mu}}\right]^{z \xi_{\mu}} \cdot g_{1}^{\sum_{j=1}^{T} z c_{j, 1}^{\prime}\left(\hat{m}_{j}-m_{j}^{*}\right)} \\
& =\frac{R^{*}}{\hat{R}}=W
\end{aligned}
$$

Note that the simulation of Game 7 provided by $\mathcal{B}$ to $\mathcal{A}$ is statistically close (by a factor $\gamma+\gamma^{\prime}$ due to the use of TrapGen in $\mathrm{H}_{\text {and }} \mathrm{H}^{\prime}$ ) to the real execution of Game 7. Then, it is easy to see that if Game 7 outputs $1, \mathcal{B}$ is able to compute the solution of the FDHI problem, as described above. In conclusion, if $\operatorname{Pr}\left[G_{7}(\mathcal{A})\right] \geq \epsilon$ then $\mathcal{B}$ has advantage at least $\left(\delta^{\prime} \epsilon\right)-\gamma-\gamma^{\prime}$ in solving FDHI.

Finally, we note that when instantiated with our $\mathrm{APHF} \mathrm{H}_{\text {sqr }}$ from Sect. 3.3, the programmability properties stated in the theorem hold with probability $\delta^{\prime}=1$ while the property of statistically-close trapdoor keys holds in a perfect sense, i.e., $\gamma=0$ and $\gamma^{\prime}=0$.

\subsection{Context-hiding security}

In this section, we prove the context-hiding security of our linearly homomorphic signature scheme.

Proof (Theorem 9) First notice that since in our case there is no hiding procedure (i.e., one can see Hide as the identity function and HVerify as Ver), correctness and unforgeability follow trivially.

In order to prove context-hiding security, we construct below a simulator and then show that its signatures are perfectly indistinguishable from the ones obtained through a run of the Eval algorithm.

Simulator $\operatorname{Sim}\left(\mathbf{s k}, \mathcal{P}_{\Delta}, \tilde{\mathbf{m}}\right)$. Parse the simulator's input as sk $=\left(\mathbf{s k}^{\prime}, K, \hat{K}\right.$, sek, sek'), $\mathcal{P}_{\Delta}=\left(\left(f_{1}, \ldots, f_{\ell}\right), \tau_{1}, \ldots, \tau_{\ell}, \Delta\right)$ and $\tilde{\mathbf{m}}=\left(\tilde{m}_{1}, \ldots, \tilde{m}_{T}\right)$. With this information, the simulator computes the following values:

- $\left\{R_{i}=g_{1}^{\mathrm{PRF} . \mathrm{KG}_{\hat{\mathrm{K}}}\left(\Delta \mid \tau_{i}\right)}\right\}_{i=1}^{\ell}$ where $\left\{\tau_{i}\right\}_{i=1}^{\ell}$ are the labels in $\mathcal{P}_{\Lambda}$.

- $\left\{\mathcal{H}_{\tau_{i}}=: \text { H.PriEval }\left(\text { sek, } \tau_{i}\right)\right\}_{i=1}^{\ell}$ and $\left\{\tilde{\mathcal{H}}_{j}=: \text { H.PriEval' }\left(\text { sek }^{\prime}, j\right)\right\}_{j=1}^{T}$.

$-Z=g_{2}^{z}$, where $z \leftarrow$ PRF.KG $\mathrm{KG}_{K}(\Delta)$.

$-\sigma_{\Delta} \leftarrow \operatorname{Sign}^{\prime}\left(\mathrm{sk}^{\prime}, \Delta \mid Z\right)$.

- $R=\prod_{i=1}^{\ell} R_{i}^{f_{i}}$. 


$$
-S=\left(\prod_{i=1}^{\ell} \mathcal{H}_{\tau_{i}}^{f_{i}} \cdot R \cdot \prod_{j=1}^{T} \tilde{\mathcal{H}}_{j}^{\tilde{m}_{j}}\right)^{1 / z} .
$$

The simulator finally outputs a signature $\sigma^{1}=\left(\sigma_{\Delta}, Z, R, S\right)$.

Indistinguishability of signatures Here we show that our simulator allows for context hiding security. Fix any choice of $(\mathrm{sk}, \mathrm{vk}) \in \operatorname{KeyGen}\left(1^{\lambda}, \mathcal{L}\right), \mathcal{P}_{\Delta}=\left(\left(f_{1}, \ldots, f_{\ell}\right), \tau_{1}, \ldots, \tau_{\ell}, \Delta\right)$, and $\mathbf{m}_{1}, \ldots, \mathbf{m}_{\ell} \in \mathcal{M}^{T}$. For all $i=1$ to $\ell$, we have $\sigma_{i}=\operatorname{Sign}\left(\mathrm{sk}, \Delta, \tau_{i}, \mathbf{m}_{i}\right)$ with $\sigma_{i}=\left(\sigma_{\Delta, i}, Z_{i}, R_{i}, S_{i}\right)$, and notice that since the scheme $\Sigma^{\prime}$ is deterministic fixing $\mathrm{sk}^{\prime}, \Delta, \tau_{1}, \mathbf{m}_{1}, \ldots, \tau_{\ell}, \mathbf{m}_{\ell}$ fixes also all the signatures $\sigma_{1}, \ldots, \sigma_{\ell}$.

Let $\sigma^{0} \leftarrow \operatorname{Eval}\left(\mathrm{vk}, \sigma_{1}, \ldots, \sigma_{\ell}\right)$ and recall that by construction the signature $\sigma^{0}=$ $\left(\sigma_{\Delta}^{\prime}, Z^{\prime}, R^{\prime}, S^{\prime}\right)$ consists of:

$-Z^{\prime}=Z_{1}=g_{2}^{z}$, where $z \leftarrow$ PRF.KG $K_{K}(\Delta)$.

$-\sigma_{\Delta}^{\prime}=\sigma_{\Delta, 1} \leftarrow \operatorname{Sign}^{\prime}\left(\mathrm{sk}^{\prime}, \Delta \mid Z\right)$.

- $R^{\prime}=\prod_{i=1}^{\ell} R_{i}^{f_{i}}$, where $R=g_{1}^{r_{i}}$ and $r_{i} \leftarrow$ PRF.KG $\hat{K}_{\hat{K}}\left(\Delta \mid \tau_{i}\right)$.

$-S^{\prime}=\prod_{i=1}^{\ell} S_{i}^{f_{i}}$.

Let us now assume that we have a distinguisher $\mathcal{D}$ which is given $I=\left(\mathrm{sk}, \mathrm{vk}, \mathcal{P}_{\Delta}, \mathbf{m}_{1}\right.$, $\left.\sigma_{1}, \ldots, \mathbf{m}_{\ell}, \sigma_{\ell}\right)$ and either one of $\sigma^{0} \leftarrow \operatorname{Eval}\left(\mathrm{vk}, f,\left\{\sigma_{i}\right\}_{i=1}^{\ell}\right)$ or $\sigma^{1} \leftarrow \operatorname{Sim}\left(s k, \mathcal{P}_{\Delta}, \tilde{\mathbf{m}}\right)$.

In what follows we show that $\sigma^{0}$ and $\sigma^{1}$ are distributed identically, that is any $\mathcal{D}$ has advantage 0 in distinguishing the two cases. To prove this, we are going to compare the two signatures $\sigma^{0}, \sigma^{1}$ element by element.

$$
\sigma^{0}=\sigma_{\Delta, 1} \quad \begin{gathered}
\sigma^{1} \\
\sigma_{\Delta} \leftarrow \operatorname{Sign}^{\prime}\left(\mathrm{sk}^{\prime}, \Delta \mid Z\right)
\end{gathered}
$$

Since the scheme $\Sigma^{\prime}$ is deterministic and by construction of $\sigma_{\Delta, 1}$ (see above), one can see that $\sigma_{\Delta}$ and $\sigma_{\Delta}^{\prime}$ are equal.

$$
Z^{\prime}=Z_{1}=g_{2}^{z} \text {, where } z \leftarrow F_{K}(\Delta) \quad \mid \quad Z=g_{2}^{z} \text {, where } z \leftarrow F_{K}(\Delta)
$$

The two elements are clearly equal because of the PRF ( $z$ is uniquely determined given $K, \Delta)$.

$$
R^{\prime}=\prod_{i=1}^{\ell} R_{i}^{f_{i}} \quad R=\prod_{i=1}^{\ell} R_{i}^{f_{i}}
$$

By the construction of the simulator the elements $\left\{R_{i}\right\}_{i=1}^{\ell}$ are the same on both sides since in both cases are generated deterministically using the PRF $F$ with seed $\hat{K}$. This implies $R=R^{\prime}$.

$$
S^{\prime}=\prod_{i=1}^{\ell} S_{i}^{f_{i}}
$$

$$
S=\left(\prod_{i=1}^{\ell} \mathcal{H}_{\tau_{i}}^{f_{i}} \cdot R \cdot \prod_{j=1}^{T} \tilde{\mathcal{H}}_{j}^{\tilde{m}_{j}}\right)^{1 / z}
$$

We claim that $S^{\prime}=S$. To see this, let us recall how each $S_{i}$ is defined: by the signing algorithm we have $S_{i}=\left(\mathcal{H}_{\tau_{i}} \cdot R_{i} \cdot \prod_{j=1}^{T} \tilde{\mathcal{H}}_{j}^{m_{i, j}}\right)^{1 / z}$. Therefore

$$
S^{\prime}=\prod_{i=1}^{\ell}\left(\mathcal{H}_{\tau_{i}}^{f_{i}} \cdot R_{i}^{f_{i}} \cdot \prod_{j=1}^{T} \tilde{\mathcal{H}}_{j}^{f_{i} m_{i, j}}\right)^{1 / z}=\left(\prod_{i=1}^{\ell} \mathcal{H}_{\tau_{i}}^{f_{i}} \cdot R \cdot \prod_{j=1}^{T} \tilde{\mathcal{H}}_{j}^{\sum_{i=1}^{\ell} f_{i} m_{i, j}}\right)^{1 / z}=S
$$

where the last equality follows from the fact that $\tilde{\mathbf{m}}=: \sum_{i=0}^{\ell} f_{i} \mathbf{m}_{i}$. 


\section{Short signatures with shorter public keys from bilinear maps}

In this section we describe how to use APHFs to construct in a generic fashion standard-model signature schemes over bilinear groups. We propose two constructions that are provablysecure under the $q$-Strong Diffie-Hellman [10] and the $q$-Diffie-Hellman [9] assumptions. These constructions are the analogues of the schemes in $[30,32]$ respectively. The basic idea behind the constructions is to replace a standard $(m, 1)$-PHF with an $(m, 1, d)$-APHF. In fact, in this context, having a secretly-computable $\mathrm{H}$ does not raise any issue when using $\mathrm{H}$ in the signing procedure as the signer already uses a secret key. At the same time, for verification purposes, computing the (public) isomorphic copy of $\mathrm{H}$ in the target group is also sufficient. Our proof confirms that the $(m, 1, d)$-programmability can still be used to control the size of the randomness in the same way as in $[30,32]$. One difference in the security proof is that the schemes in $[30,32]$ are based on the $q$-(S)DH assumption, where $q$ is the number of signing queries made by the adversary, whereas ours have to rely on the $(q+d-1)$-(S)DH problem. Since our instantiations use $d=2$, the difference (when considering concrete security) is very minor.

When plugging into these generic constructions our new APHF, $\mathrm{H}_{\mathrm{acts}}$, described in Sect. 3.2, which is $(m, 1,2)$-programmable, we obtain schemes that, for signing $\ell$-bits messages, allow for public keys of length $O(m \sqrt{\ell})$ as in [39].

We describe the scheme based on $q-\mathrm{SDH}$ in Sect.5.1, and the one based on $q-\mathrm{DH}$ in Sect. 5.2. As discussed in [32], the advantage of the scheme from $q$-DH compared to the one from $q-\mathrm{SDH}$ is to be based on a weaker assumption.

\subsection{A $q$-strong Diffie-Hellman based solution}

In this section we revisit the $q$-SDH based solution of [30]. The signature $\Sigma_{\mathrm{qSDH}}=$ (KeyGen, Sign, Ver) is as follows:

KeyGen $\left(1^{\lambda}\right)$. Let $\lambda$ be the security parameter, and let $\ell=\ell(\lambda)$ and $\rho=\rho(\lambda)$ be arbitrary polynomials. Our scheme can sign messages in $\{0,1\}^{\ell}$ using randomness in $\{0,1\}^{\rho}$. The key generation algorithm works as follows:

- Run bgp $\stackrel{\$}{\leftarrow} \mathcal{G}\left(1^{\lambda}\right)$ to generate the bilinear groups parameters bgp $=\left(p, \mathbb{G}_{1}, \mathbb{G}_{2}, \mathbb{G}_{T}\right.$, $\left.g_{1}, g_{2}, e\right)$ where $\mathbb{G}_{1}, \mathbb{G}_{2}$ and $\mathbb{G}_{T}$ are asymmetric groups of prime order $p \approx 2^{\lambda}, g_{1} \in \mathbb{G}_{1}$, $g_{2} \in \mathbb{G}_{2}$ are generators and $e: \mathbb{G}_{1} \times \mathbb{G}_{2} \rightarrow \mathbb{G}_{T}$ is an efficiently computable, nondegenerate bilinear map.

- Run (sek, pek) $\stackrel{\$}{\leftarrow}$ H.Gen $\left(1^{\lambda}\right.$, bgp) to generate the keys of the asymmetric hash function.

- Choose a random $x \stackrel{\$}{\leftarrow} \mathbb{Z}_{p}^{*}$ and set $X \leftarrow g_{2}^{x}$. Return vk $=$ (bgp, pek, $X$ ) and sk $=$ (sek, $x$ ).

Sign (sk, $M$ ). The signing algorithm takes as input the secret key sk, and a message $M \in\{0,1\}^{\ell}$. It starts by generating a random $r \stackrel{\$}{\leftarrow}\{0,1\}^{\rho}$. Next, it computes $\sigma=$ H.PriEval(sek, $M)^{\frac{1}{x+r}}$ and outputs $(\sigma, r)$.

$\operatorname{Ver}(\mathrm{vk}, M,(\sigma, r))$. To check that $(\sigma, r)$ is a valid signature, check that $r$ is of length $\rho$ and that

$$
e\left(\sigma, X \cdot g_{2}^{r}\right)=\text { H.PubEval }(\text { pek, } M)
$$

We state the security of the scheme in the following theorem (whose proof appears in the full version). We note that for simplicity our proof assumes an $(m, 1, d)$-APHF for $d=2$, which 
matches our realization. A generalization of the theorem for a generic $d$ can be immediately obtained, in which case one would rely on the $(q+d-1)$-SDH assumption.

Theorem 12 Assume that $\mathcal{G}$ is a bilinear group generator such that the $(q+1)$-SDH assumption holds in $\mathbb{G}_{1}$ and $\mathrm{H}$ is $(m, 1,2, \gamma, \delta)$-programmable, then $\Sigma_{\mathrm{qSDH}}$ is a secure signature scheme. More precisely, let $\mathcal{B}$ be an efficient (probabilistic) algorithm that runs in time $t$, asks (up to) q signing queries and produces a valid forgery with probability $\epsilon$, then there exists an equally efficient algorithm.A that confutes the $(q+1)-S D H$ assumption with probability

$$
\epsilon^{\prime} \geq \frac{\delta}{q}\left(\epsilon-\gamma-\frac{q}{p}-\frac{q^{m+1}}{2^{\rho m}}\right)
$$

Proof This proof is almost identical to the corresponding one from [30], we rewrite it here mainly to show how to use APHFs in place of standard PHFs.

Let $\mathcal{B}$ be an adversary against the signature scheme. Assuming that $\mathcal{B}$ asks (up to) $q$ signing queries, and denote with $M_{i}$ the $i$-th queried message and with $\left(\sigma_{i}, r_{i}\right)$ the corresponding signature. Also, let $M^{*},\left(\sigma^{*}, r^{*}\right)$ be the produced forgery. We distinguish two types of forgeries:

Type I forgery: It holds that $r^{*}=r_{i}$ for some $i \in[q]$.

Type II forgery: It holds that $r^{*} \neq r_{i} \forall i \in[q]$.

Notice that these two cases are mutually exclusive and completely cover the set of possible forgeries. Now we show that both types of forgeries can be used to violate the $(q+1)$-SDH assumption.

Lemma 10 (Type I forgeries) Let $\mathcal{B}$ be a type I forger that breaks the signature scheme with advantage $\epsilon_{1}$ (and making up to $q$ signature queries). Then there exists an (equally efficient) adversary $\mathcal{A}$ that breaks the $(q+1)-S D H$ assumption with advantage $\epsilon^{\prime}$, where

$$
\epsilon^{\prime} \geq \frac{\delta}{q}\left(\epsilon_{1}-\gamma-\frac{q}{p}-\frac{q^{m+1}}{2^{\rho m}}\right)
$$

We prove the lemma via a sequence of games. We denote with $G_{i}$ the event that Game $i$ outputs 1, i.e., that $\mathcal{B}$ (successfully) forges in Game $i$.

Game 0 This game is the standard existential unforgeability experiment $\operatorname{Exp}_{\mathcal{B}, \Sigma}^{\text {UF-CMA }}$. Clearly,

$$
\operatorname{Pr}\left[G_{0}\right]=\epsilon_{1}
$$

Game 1 This is the same as the previous game but the parameter of the APHF are generated using H.TrapGen (rather than H.Gen). More precisely, $\mathcal{A}$ runs H.TrapGen $\left(1^{\lambda}, g_{1}\right.$, $h_{1}, g_{2}, h_{2}$ ), where $g_{1}, g_{2}$ are, randomly chosen, generators and $h_{1}=g_{1}^{\alpha}, h_{2}=g_{2}^{\alpha}$ for a randomly chosen $\alpha \stackrel{\$}{\leftarrow} \mathbb{Z}_{p}$. By the $\gamma$-closeness of the trapdoor keys, we have:

$$
\operatorname{Pr}\left[G_{1}\right] \geq \operatorname{Pr}\left[G_{0}\right]-\gamma
$$

Game 2 In this game we do the following changes. First, we choose the $r_{i}$ 's used to answer signing queries all in advance (rather than one by one when needed). Since the $r_{i}$ 's are chosen at random and independently anyway this change cannot affect $\mathcal{B}$ 's advantage at all. Second, we modify the way $g_{1}, h_{1}, g_{2}, h_{2}$ are chosen when executing H.TrapGen. Specifically, let $g_{1}$ be a generator of $\mathbb{G}_{\wedge}$ and $g_{2}$ be a generator of $\mathbb{G}_{2}$. We choose $i^{*} \in_{R}$

$\{1, \ldots, q\}$ and we set $r^{*}=r_{i *}, R=\cup_{i=1}^{q} r_{i}, R^{*}=R \backslash\left\{r^{*}\right\}$ 
and $R^{*, i}=R \backslash\left\{r^{*}, r_{i}\right\}$. Next, we define the polynomials $p^{*}(z)=\prod_{r \in R^{*}}(z+$ $r) \bmod p$ and $p(z)=p^{*}(z)\left(z+r^{*}\right) \bmod p$. Notice that both polynomials are of degree $\leq q$. Thus, from $\hat{g}_{1}, \hat{g}_{1}^{x}, \ldots, \hat{g}_{1}^{x^{q}}$ it is possible to compute $g_{1}=\hat{g}_{1}^{p^{*}(x)}$ and $h_{1}=\hat{g}_{1}^{p(x)}$. Next we set $g_{2}=\hat{g}_{2}, X=g_{2}^{x}$ and $h_{2}=g_{2}^{\left(x+r^{*}\right)}$. The distribution of $g_{1}, g_{2}$ is identical to the one in Game 1 . The only difference might occur in the case $p(x)=0$, as in this case $g_{1}, h_{1}$ would not be generators. By the Schwartz-Zippel lemma $[37,40]$, however, this happens only with probability at most $q / p$. Thus

$$
\operatorname{Pr}\left[G_{2}\right] \geq \operatorname{Pr}\left[G_{1}\right]-\frac{q}{p}
$$

Game $3 \mathrm{Let} \mathrm{Bad}_{3}$ be the event that the same $r_{i}$ is used to sign more than $m$ different messages. This means that if $\mathrm{Bad}_{3}$ occurs there are, at least, $m+1$ indices $i_{1}, \ldots, i_{m+1}$ such that $r_{i_{1}}=\ldots=r_{i_{m+1}}$. On $q$ signing queries there might be up to $\left(\begin{array}{c}q \\ m+1\end{array}\right) \leq q^{m+1}$ such tuples. Moreover, a given tuple is of the form $r_{i_{1}}=\ldots=r_{i_{m+1}}$ with probability $2^{\rho} / 2^{\rho(m+1)}$. This means that

$$
\operatorname{Pr}\left[\mathrm{Bad}_{3}\right] \leq \frac{q^{m+1}}{2^{\rho m}}
$$

We modify Game 2, by letting that the simulation aborts if $\mathrm{Bad}_{3}$ occurs. Thus,

$$
\operatorname{Pr}\left[G_{3}\right] \geq \operatorname{Pr}\left[G_{2}\right]-\frac{q^{m+1}}{2^{\rho m}}
$$

Game 4 Let $\mathrm{Bad}_{4}$ be the event that $\mathcal{B}$ outputs a value $r^{*}$ such that $r^{*}=r_{i}$, but $i \neq i^{*}$. We modify the previous game by imposing that the simulation aborts if $\mathrm{Bad}_{4}$ occurs. Thus,

$$
\operatorname{Pr}\left[G_{4}\right]=\operatorname{Pr}\left[G_{4} \wedge \neg \mathrm{Bad}_{4}\right]=\frac{1}{q} \operatorname{Pr}\left[G_{3}\right]
$$

Game 5 Let $\mathrm{Bad}_{5}$ be the event that either there is an index $i \in[q]$ such that $r_{i}=r^{*}$ such that $c_{M_{i}, 0} \neq 0$, or it occurs $c_{M^{*}, 0}=0$. Game 5 proceeds as Game 4 except that it aborts if $\mathrm{Bad}_{5}$ occurs. Using the programmability of $\mathrm{H}$, we can bound the probability of $\mathrm{Bad}_{5}$. Precisely, we have that $\operatorname{Pr}\left[\neg \mathrm{Bad}_{5}\right] \geq \delta$, from which we have

$$
\operatorname{Pr}\left[G_{5}\right]=\operatorname{Pr}\left[G_{5} \wedge \neg \mathrm{Bad}_{5}\right]=\delta \operatorname{Pr}\left[G_{4}\right]
$$

Game 6 We further modify the simulation by using the alternative signing mechanism, from [10]. In particular, to sign the $i$-th queried message $M_{i}$, one proceeds as follows. First, compute $\mathbf{c}_{M_{i}} \leftarrow \mathrm{H}$. TrapEval(td, $M_{i}$ ). Notice that by the setting of $h_{1}, h_{2}$ and by the definition of TrapEval, $\mathbf{c}_{M_{i}}$ is a degree-2 polynomial $c_{M_{i}}\left(x+r^{*}\right)$. Let us write $c_{M_{i}}\left(x+r^{*}\right)=c_{M_{i}, 0}+c_{M_{i}}^{\prime}\left(x+r^{*}\right)$, where $c_{M_{i}}^{\prime}\left(x+r^{*}\right)$ is the degree-2 polynomial obtained by simply removing the degree-0 term from $c_{M_{i}}\left(x+r^{*}\right)$. Hence, one computes

$$
\begin{aligned}
\sigma_{i} & =\text { H.PriEval }\left(\text { sek, } M_{i}\right)^{\frac{1}{x+r_{i}}}=\left(g_{1}^{c_{M_{i}, 0}+c_{M_{i}}^{\prime}\left(x+r^{*}\right)}\right)^{\frac{1}{x+r_{i}}} \\
& =\left(\hat{g}_{1}^{p^{*}(x) c_{M_{i}, 0}} \hat{g}_{1}^{\left.p^{*}(x) c_{M_{i}^{\prime}}^{\left(x+r^{*}\right)}\right)^{\frac{1}{x+r_{i}}}}\right. \\
& =\hat{g}_{1}^{c_{M_{i}, 0} \prod_{r \in R^{*, i}}(x+r)} \hat{g}_{1}^{c_{M_{i}}^{\prime}\left(x+r^{*}\right) \prod_{r \in R^{*, i}}(x+r)}
\end{aligned}
$$


Moreover, for all the signing queries that do not cause $\mathrm{Bad}_{5}$, notice that $c_{M_{i}, 0}=0$ and thus such signing queries can be answered without any explicit knowledge of $x$. As a consequence,

$$
\operatorname{Pr}\left[G_{6}\right]=\operatorname{Pr}\left[G_{5}\right]
$$

Notice also that in Game 6, we are assuming that neither $\operatorname{Bad}_{5}$ nor $\operatorname{Bad}_{4}$ occur. This means that, for the the forged signature $\left(M^{*}, \sigma^{*}, r^{*}\right)$ one has that H.PriEval(sek, $\left.M^{*}\right)=g_{1}^{c_{M^{*}}\left(x+r^{*}\right)}$ and $c_{M^{*}, 0} \neq 0$. Using the same notation as above, using the $q$-SDH instance we can compute

$$
y=g_{1}^{\frac{c_{m_{i}}^{\prime}\left(x+r^{*}\right)}{x+r^{*}}}
$$

as $c_{m_{i}}^{\prime}\left(x+r^{*}\right)$ is a polynomial of degree $\leq 2$ without the constant term, i.e, $c_{m_{i}}^{\prime}(x+$ $\left.r^{*}\right)$ is divisible by $\left(x+r^{*}\right)$. We set

$$
\sigma^{\prime}=\left(\sigma^{*} \cdot y^{-1}\right)^{1 / c_{m^{*}, 0}}=g_{1}^{\frac{1}{x+r^{*}}}=\hat{g}_{1}^{\frac{p^{*}(x)}{x+r^{*}}}
$$

Using standard techniques $[10,30], \sigma^{\prime}$ can be used to extract the required $\hat{g}_{1}^{\frac{1}{x+r^{*}}}$. This means that $\operatorname{Pr}\left[G_{6}\right] \leq \epsilon$. Finally, putting together the bounds from the games above yields the lemma.

Lemma 11 (Type II forgeries) Let $\mathcal{B}$ be a type II forger that breaks the signature scheme with advantage $\epsilon_{2}$ (and making up to $q$ signature queries). Then there exist (equally efficient) adversaries $\mathcal{A}_{1}$, that breaks the $(q+1)-S D H$ assumption with advantage $\epsilon$, and $\mathcal{A}_{2}$ that breaks the discrete logarithm problem with advantage $\epsilon_{\mathrm{DL}}$, where

$$
\epsilon+\epsilon_{\mathrm{DL}} \geq \epsilon_{2}-q / p-\gamma
$$

Again we prove the lemma via a sequence of games, and use $G_{i}$ to denote the event that $\mathcal{B}$ (successfully) forges in Game $i$.

Game 0 This game is the standard existential unforgeability experiment $\operatorname{Exp}_{\mathcal{B}, \Sigma}^{\mathrm{UF}-\mathrm{CMA}}$. Clearly,

$$
\operatorname{Pr}\left[G_{0}\right]=\epsilon_{2}
$$

Game 1 This is the same as Game 1 above, (i.e. the parameter of the programmable hash function are generated using H.TrapGen (rather than H.Gen). Thus,

$$
\operatorname{Pr}\left[G_{1}\right] \geq \operatorname{Pr}\left[G_{0}\right]-\gamma
$$

Game 2 In this game we do the following changes. First, we choose the $r_{i}$ 's used to answer signing queries all in advance (rather than one by one when needed). Second, we modify the way $g_{1}, h_{1}, g_{2}, h_{2}$ are chosen when executing $H$. TrapGen. Specifically, let $\hat{g}_{1}$ be a generator of $\mathbb{G}_{1}$ and $\hat{g}_{2}$ be a generator of $\mathbb{G}_{2}$. We we set $R=\cup_{i=1}^{q} r_{i}$. Next we define the $($ degree- $q)$ polynomial $p(z)=\prod_{r \in R}(z+r) \bmod p$. From $\hat{g}_{1}, \hat{g}_{1}^{x}, \ldots, \hat{g}_{1}^{x^{q}}$ it is possible to compute $g_{1}=\hat{g}_{1}^{p(x)}$ and $h_{1}=g_{1}=\hat{g}_{1}^{p(x)}$. Next we set $g_{2}=\hat{g}_{2}, X=g_{2}^{x}$, and $h_{2}=g_{2}^{\alpha}$ (for random $\alpha \stackrel{\$}{\leftarrow} \mathbb{Z}_{p}$ ). Note that the distribution of $g_{1}, g_{2}$ is identical with respect to Game 1 . Again, the only difference might occur in the case when $p(x)=0$, as in this case $g_{1}$ would not be a generator. Thus

$$
\operatorname{Pr}\left[G_{2}\right] \geq \operatorname{Pr}\left[G_{1}\right]-\frac{q}{p}
$$


Game 3 Let $M^{*}$ be the message used in the forgery, and let $c_{M^{*}} \leftarrow \mathrm{H}$. TrapEval(td, $\left.M^{*}\right)$. We define $\mathrm{Bad}_{3}$ as the event that $c_{M^{*}}(\alpha)=0$. Then, if $\mathrm{Bad}_{3}$ happens Game 3 aborts. It is not hard to show, that if $\mathrm{Bad}_{3}$ occurs, then one can break the discrete log assumption in the group $\mathbb{G}_{2}$ (which in turn is implied by the $(q+1)$-SDH assumption). Indeed, $c_{M^{*}}(\alpha)=c_{M^{*}, 0}+c_{M_{1}^{*} \alpha}$ is here a degree-1 polynomial in the variable $\alpha$, and the elements $g_{2}, g_{2}^{\alpha}$ are given as part of a challenge $\left(g_{1}, g_{1}^{\alpha}, \ldots, g_{1}^{\alpha^{q}}, g_{2}, g_{2}^{\alpha}\right)$ of our $(q+1)-\mathrm{SDH}$ assumption. The unknown discrete $\log \alpha$ can be then easily computed as $\alpha=-c_{M^{*}, 0} / c_{M^{*}, 1}$.

$$
\operatorname{Pr}\left[G_{3}\right] \geq \operatorname{Pr}\left[G_{2}\right]-\epsilon_{\mathrm{DL}}
$$

Game 4 We further modify the simulation by using the alternative signing mechanism, from [10]. In particular, to sign the $i$-th message $M_{i}$ one obtains $c_{M_{i}} \leftarrow \mathrm{H}$. TrapEval $\left(\mathrm{td}, M_{i}\right)$ and then computes

$$
\begin{aligned}
\sigma_{i} & =\text { H.PriEval }\left(\text { sek, } M_{i}\right)^{\frac{1}{x+r_{i}}}=\left(g_{1}^{c_{M_{i}}(\alpha)}\right)^{\frac{1}{x+r_{i}}}=\left(\hat{g}_{1}^{p(x) c_{M_{i}}(\alpha)}\right)^{\frac{1}{x+r_{i}}} \\
& =\hat{g}_{1}^{c_{M_{i}}(\alpha) \prod_{\left.r \in R \backslash r_{i}\right\}}(x+r)}
\end{aligned}
$$

Since all the signing queries, can be answered without any explicit knowledge of $x$ we have that

$$
\operatorname{Pr}\left[G_{4}\right]=\operatorname{Pr}\left[G_{3}\right]
$$

Notice also that since we are assuming that $\mathrm{Bad}_{3}$ does not occur we have that, from the produced forgery on $M^{*}$ we can extract

$$
\sigma^{\prime}=\left(\sigma^{*}\right)^{1 / c_{M^{*}}(\alpha)}=g_{1}^{\frac{1}{x+r^{*}}}=\hat{g}_{1}^{\frac{p(x)}{x+r^{*}}}
$$

Again, by using standard techniques $[10,30], \sigma^{\prime}$ can be used to extract the required $\hat{g}_{1}^{\frac{1}{x+r^{*}}}$. Hence, $\operatorname{Pr}\left[G_{4}\right] \leq \epsilon$. Finally, putting together the bounds from the games above yields the lemma.

\subsection{A $q$-Diffie-Hellman based solution}

In this section we show how to revisit the $q$-DH based scheme of [32] in order to work with APHFs. Our construction uses a standard PHF as an additional building block. We construct a signature $\Sigma_{\mathrm{qDH}}=$ (KeyGen, Sign, Ver) as follows:

KeyGen $\left(1^{\lambda}\right)$. Let $\lambda$ be the security parameter, and let $\ell=\ell(\lambda)$ and $\rho=\rho(\lambda)$ be arbitrary polynomials. The scheme can sign messages in $\{0,1\}^{\ell}$ using randomness in $\{0,1\}^{\rho}$. The key generation algorithm works as follows:

- Run bgp $\stackrel{\$}{\leftarrow} \mathcal{G}\left(1^{\lambda}\right)$ to generate the bilinear groups parameters bgp $=\left(p, \mathbb{G}_{1}, \mathbb{G}_{2}, \mathbb{G}_{T}\right.$, $\left.g_{1}, g_{2}, e\right)$ where $\mathbb{G}_{1}, \mathbb{G}_{2}$ and $\mathbb{G}_{T}$ are groups of prime order $p \approx 2^{\lambda}, g_{1} \in \mathbb{G}_{1}, g_{2} \in \mathbb{G}_{2}$ are generators and $e: \mathbb{G}_{1} \times \mathbb{G}_{2} \rightarrow \mathbb{G}_{T}$ is an efficiently computable, non-degenerate bilinear map.

- Run (sek, pek) $\stackrel{\$}{\leftarrow}$ H.Gen $\left(1^{\lambda}\right.$, bgp) to generate the keys of the asymmetric hash function.

- Let $D=$ (PHF.Gen, PHF.Eval) be a group hash function [30] over $\mathbb{G}_{2}$ with input length $\rho$ such that $D$ is programmable using the algorithms (PHF. TrapGen, PHF.TrapEval). Run $(\kappa, \tau) \leftarrow$ PHF. TrapGen $\left(1^{\lambda}, g_{1}, g_{1}^{y}\right)$, for a random $y \stackrel{\$}{\leftarrow} \mathbb{Z}_{p}$. 
- Return vk $=(\operatorname{bgp}$, pek, $\kappa)$ and $\mathrm{sk}=(\mathrm{sek}, \tau, y)$. In what follows, we use the same notation of [32], and use $\mathrm{d}(r)$ as a shorthand for $(a, b) \leftarrow \operatorname{PHF}$.TrapEval $(\tau, r), \mathrm{d}(r)=$ $a+y b$.

Sign(sk, $M$ ). The signing algorithm takes as input the secret key sk, and a message $M \in\{0,1\}^{\ell}$. It starts by generating a random $r \in\{0,1\}^{\rho}$. Next, it computes $\sigma=\mathrm{H}$.PriEval (sek, $M)^{\frac{1}{\mathrm{~d}(r)}}$ and outputs $(\sigma, r)$.

$\operatorname{Ver}(\mathrm{vk}, M,(\sigma, r))$. To verify that $(\sigma, r)$ is a valid signature, check that $r$ is of length $\rho$, that $\mathrm{d}(r) \neq 0$ and that

$$
e(\sigma, \operatorname{PHF} . \operatorname{Eval}(r))=\mathrm{H} . \operatorname{PubEval}(\text { pek, } M)
$$

We prove the security of the scheme in the following theorem. We note that for simplicity our proof assumes an $(m, 1, d)$-APHF for $d=2$, which matches our realization. A generalization of the theorem for a generic $d$ can be immediately obtained, in which case one would rely on the $(q+d-1)$-DH assumption.

Theorem 13 Assume that $\mathcal{G}$ is a bilinear group generator such that the $(q+1)$-DH assumption holds in $\mathbb{G}_{1}, \mathrm{H}$ is an asymmetric $(m, 1,2, \gamma, \delta)$-programmable hash function, $\mathrm{D}$ is a (1, poly, $\left.\gamma^{\prime}, \delta^{\prime}\right)$ programmable hash function over $\mathbb{G}_{2}$ then $\Sigma_{\mathrm{qDH}}$ is a secure signature scheme. More precisely let $\mathcal{B}$ be an efficient (probabilistic) algorithm that runs in time t, asks (up to) $q$ signing queries and produces a valid forgery with probability $\epsilon_{1}$, then there exists an equally efficient algorithm $\mathcal{A}$ that confutes the $(q+1)$-DH assumption with probability

$$
\epsilon^{\prime} \geq \delta \delta^{\prime}\left(\frac{\epsilon_{1}}{q}-\gamma-\frac{q^{m}}{2^{\rho m}}\right)
$$

Proof Again the proof is almost identical to the corresponding one from [30], we rewrite it here for completeness. Let $\mathcal{B}$ be an adversary against the signature scheme. Assuming that $\mathcal{B}$ asks (up to) $q$ signing queries we denote with $M_{i}$ the $i$-th queried message and with $\left(\sigma_{i}, r_{i}\right)$ the corresponding signature. Also, let $M^{*},\left(\sigma^{*}, r^{*}\right)$ be the produced forgery. We distinguish two types of forgeries

Type I forgery : It holds that $r^{*}=r_{i}$ for some $i \in[q]$.

Type II forgery : It holds that $r^{*} \neq r_{i} \forall i \in[q]$.

Notice that these two cases are mutually exclusive and completely cover the set of possible forgeries. Now we show that both types of forgeries can be used to violate the $(q+1)$-DH assumption.

Lemma 12 (Type I forgeries) Let $\mathcal{B}$ be a type I forgery that breaks the signature scheme with advantage $\epsilon_{1}$ (and making up to $q$ signature queries). Then there exists an (equally efficient) adversary $\mathcal{A}$ that breaks the $(q+1)-D H$ assumption with advantage $\epsilon^{\prime}$, where

$$
\epsilon^{\prime} \geq \delta \delta^{\prime}\left(\frac{\epsilon_{1}}{q}-\gamma-\frac{q^{m}}{2^{\rho m}}\right)
$$

Again we prove the lemma via a sequence of games, and use $G_{i}$ to denote the event that $\mathcal{B}$ (successfully) forges in Game $i$.

Game 0 This game is the standard existential unforgeability experiment $\operatorname{Exp}_{\mathcal{B}, \Sigma}^{\mathrm{UF}-\mathrm{CMA}}$. Clearly,

$$
\operatorname{Pr}\left[G_{0}\right]=\epsilon_{1}
$$


Game 1 Let $\mathrm{Bad}_{1}$ be the event that the same $r_{i}$ is used more than $m$ times. We change the simulation by forcing an abort if $\mathrm{Bad}_{1}$ occurs. As done in Lemma 10 we have that

$$
\operatorname{Pr}\left[G_{1}\right] \geq \operatorname{Pr}\left[G_{0}\right]-\frac{q^{m+1}}{2^{\rho m}}
$$

Game 2 In this game we do the following changes. First, we choose the $r_{i}$ 's used to answer signing queries all in advance (rather than one-by-one when needed). Since the $r_{i}$ 's are chosen randomly and independently anyway, this change cannot affect $\mathcal{B}$ 's advantage at all. Second, we guess the index $i$ such that $i=i^{*}$ and we abort if this does not happen (i.e. $\mathcal{B}$ outputs an $r^{*} \neq r_{i}$ ). Clearly,

$$
\operatorname{Pr}\left[G_{2}\right] \geq \frac{1}{q} \operatorname{Pr}\left[G_{1}\right]
$$

Game 3 In this game we do the following changes. First, the parameter of the asymmetric programmable hash function are generated using H.TrapGen (rather than H.Gen). Next, we modify the way $g_{1}, h_{1}, g_{2}, h_{2}$ are chosen when executing H.TrapGen. Specifically, let $\hat{g}_{1}$ be a generator of $\mathbb{G}_{1}$ and $\hat{g}_{2}$ be a generator of $\mathbb{G}_{2}$. Let $R=\cup_{i=1}^{q} r_{i}$, $R^{*}=R \backslash\left\{r^{*}\right\}$ and $R^{*, i}=R \backslash\left\{r^{*}, r_{i}\right\}$. We set

$$
g_{1}=\hat{g}_{1}^{\prod_{r \in R^{*}} \mathrm{~d}(r)} \quad h_{1}=\hat{g}_{1}^{\prod_{r \in R} \mathrm{~d}(r)} \quad g_{2}=\hat{g}_{2} \quad h_{2}=\hat{g}_{2} \mathrm{~d}\left(r^{*}\right)
$$

The $\gamma$-statistical closeness of H's trapdoor keys implies

$$
\operatorname{Pr}\left[G_{3}\right] \geq \operatorname{Pr}\left[G_{2}\right]-\gamma
$$

Game 4 Let $\mathrm{Bad}_{4}$ be the event that, letting $\mathbf{c}_{M_{i}} \leftarrow \mathrm{H}$. TrapEval(td, $M_{i}$ ), the following happens. Either $c_{M_{i}, 0} \neq 0$ for some $i$ for which $r_{i}=r^{*}$, or $c_{M^{*}, 0}=0$ (where $\mathbf{c}_{M^{*}} \leftarrow \mathrm{H}$. TrapEval(td, $\left.M^{*}\right)$ ). Game 4 proceeds as Game 3 except that it it aborts if $\mathrm{Bad}_{4}$ occurs. The programmability of $\mathrm{H}$ implies that

$$
\operatorname{Pr}\left[G_{4}\right]=\operatorname{Pr}\left[G_{3} \wedge \neg \mathrm{Bad}_{4}\right] \geq \delta \operatorname{Pr}\left[G_{3}\right]
$$

Game 5 Now we change the way signing queries are answered. Whenever a message $M_{i}$ is queried, the simulator computes $c_{M_{i}} \leftarrow \mathrm{H}$. TrapEval(td, $\left.m_{i}\right)$ and sets

$$
\sigma_{i}=\hat{g}_{1}^{c_{M_{i}}\left(\mathbf{d}\left(r^{*}\right)\right) \prod_{r \in R^{+, i}} \mathrm{~d}(r)}
$$

Notice that it is possible to sign all the received signing queries as, by Game 4, for all $r_{i}=r^{*}$, it holds $c_{M_{i}, 0}=0$. Game 5 is perfectly indistinguishable from Game 4 , from $\mathcal{B}$ 's perspective, i.e.,

$$
\operatorname{Pr}\left[G_{5}\right]=\operatorname{Pr}\left[G_{4}\right]
$$

Game 6 Now for each $r_{i}$ we compute $\left(a_{i}, b_{i}\right) \leftarrow$ PHF.TrapEval $\left(\tau, r_{i}\right)$ (for the received forgery we would get $\left.\left(a^{*}, b^{*}\right)\right)$. Let $\operatorname{Bad}_{6}$ be the event that, either $a_{i} \equiv 0 \bmod p$ for some $i$ such that $r^{*}=r_{i}$, or $a^{*} \neq 0$. If Bad $_{6}$ occurs, Game 6 aborts. By the (1, poly, $\left.\gamma^{\prime}, \delta^{\prime}\right)$ programmability of $\mathrm{D}$

$$
\operatorname{Pr}\left[G_{6}\right] \geq \delta^{\prime} \operatorname{Pr}\left[G_{5}\right]
$$

Now we embed the received $(q+1)$-DH challenge $\left(\hat{g}_{1}, \hat{g}_{1}^{y}, \ldots, \hat{g}_{1}^{y^{q+1}}, \hat{g}_{2}, \hat{g}_{2}^{y}\right)$ as input, and we proceed as before (but using the fact that we do not explicitly know $y$ ). It is easy to check that all signing queries can be answered. Moreover, once 
the forgery $\left(M^{*}, \sigma^{*}, r^{*}\right)$ is produced, we can extract a solution of the $(q+1)$-DH challenge as follows. First, since by Game $4 c_{M^{*}, 0} \neq 0$, we can write

$$
z=\left(\frac{\sigma^{*}}{\hat{g}_{1}^{c^{\prime} M^{*}\left(\mathrm{~d}\left(r^{*}\right)\right)} \prod_{r \in R^{*}}\left(a_{r}+y b_{r}\right)}\right)^{b^{*} / c_{M^{*}, 0}}
$$

where, $c_{M_{*}}^{\prime}\left(\mathrm{d}\left(r^{*}\right)\right)$ is the polynomial obtained from $c_{M^{*}}\left(\mathrm{~d}\left(r^{*}\right)\right)$ by removing the constant term $c_{M^{*}, 0}$ and dividing by $\left.\mathrm{d}\left(r^{*}\right)\right)$.

$$
\begin{aligned}
z & =\left(\frac{\text { H.PriEval }\left(\text { sek, } M^{*}\right)^{\frac{1}{\mathrm{~d}\left(r^{*}\right)}}}{\hat{g}_{1}^{c_{M^{*}}^{\prime}\left(\mathrm{d}\left(r^{*}\right)\right) \prod_{r \in R^{*}}\left(a_{r}+y b_{r}\right)}}\right)^{b^{*} / c_{M^{*}, 0}}=\left(\frac{g_{1}^{c_{M^{*}}\left(\mathrm{~d}\left(r^{*}\right)\right) / \mathrm{d}\left(r^{*}\right)}}{\hat{g}_{1}^{c_{M^{*}}\left(\mathrm{~d}\left(r^{*}\right)\right) \prod_{r \in R^{*}}\left(a_{r}+y b_{r}\right)}}\right)^{b^{*} / c_{m^{*}, 0}} \\
& =\left(\frac{g_{1}^{c_{M^{*}, 0} / \mathrm{d}\left(r^{*}\right)} \hat{g}_{1}^{c_{M^{*}}^{\prime}\left(\mathrm{d}\left(r^{*}\right)\right) \prod_{r \in R^{*}}\left(a_{r}+y b_{r}\right)}}{\hat{g}_{1}^{c^{\prime} M^{*}}\left(\mathrm{~d}\left(r^{*}\right)\right) \prod_{r \in R^{*}}\left(a_{r}+y b_{r}\right)}\right)^{b^{*} / c_{M^{*}, 0}}=\left(g_{1}^{c_{M^{*}, 0} / \mathrm{d}\left(r^{*}\right)}\right)^{b^{*} / c_{M^{*}, 0}} \\
& =\left(g_{1}^{c_{M^{*}, 0} /\left(y b^{*}\right)}\right)^{b^{*} / c_{M^{*}, 0}}=g_{1}^{1 / y}
\end{aligned}
$$

Finally, by using techniques from [10] one can easily get the desired result $\hat{g}_{1}^{1 / y}$.

Lemma 13 (Type II forgeries) Let $\mathcal{B}$ be a type II forger that breaks the signature scheme with advantage $\epsilon_{2}$ (and making up to $q$ signature queries). Then there exists an (equally efficient) adversary $\mathcal{A}$ that breaks the $(q+1)-D H$ assumption with advantage $\epsilon^{\prime}$ and an adversary that breaks the discrete logarithm assumption with advantage $\epsilon^{\prime \prime}$ where

$$
\epsilon^{\prime}+\delta^{\prime} \epsilon^{\prime \prime} \geq \delta^{\prime}\left(\epsilon_{2}-\gamma\right)
$$

This lemma can be proved by easily adapting the proof of Lemma 11 to this setting.

Acknowledgements The research of Dario Fiore and Luca Nizzardo is partially supported by the Spanish Ministry of Economy under Project References TIN2015-70713-R (DEDETIS), RTC-2016-4930-7 (DataMantium), and by the Madrid Regional Government under Project N-Greens (Ref. S2013/ICE-2731). Dario Fiore is also supported by a Juan de la Cierva fellowship from the Spanish Ministry of Economy.

\section{Appendix A: Digital signatures}

A digital signature scheme consists of three algorithms $\Sigma=$ (KeyGen, Sign, Ver) such that:

$\operatorname{KeyGen}\left(1^{\lambda}\right)$ the key generation takes as input a security parameter $\lambda$ and returns a secret key sk and a public verification key vk.

Sign(sk, $m$ ) on input a secret key sk and a message $m$, the signing algorithm generates a signature $\sigma$.

$\operatorname{Ver}(\mathrm{vk}, m, \sigma)$ given a triple $\mathrm{vk}, m, \sigma$ the verification algorithm outputs 1 (accept) if $\sigma$ is a valid signature on $m$ for verification key $v k$, and 0 (reject) otherwise.

The security of a signature scheme, called existential unforgeability against chosen message attacks (UF-CMA) is defined via the following experiment: 
Experiment $\operatorname{Exp}_{\mathcal{A}, \Sigma}^{\mathrm{UF}-\mathrm{CMA}}(\lambda)$

(sk, vk) $\stackrel{\$}{\leftarrow}$ KeyGen $\left(1^{\lambda}\right)$

$\left(m^{*}, \sigma^{*}\right) \stackrel{\$}{\leftarrow} \mathcal{A}^{\text {Sign }(\mathrm{sk}, \cdot)}(\mathrm{vk})$

If Ver(vk, $\left.m^{*}, \sigma^{*}\right)=1$ and $m^{*} \neq m_{i}$ for all $m_{i}$ queried to Sign(sk, $\left.\cdot\right)$, output 1

Else Output 0

The advantage of $\mathcal{A}$ in breaking the UF-CMA-security of $\Sigma$ is $\operatorname{Adv}_{\mathcal{A}, \Sigma}^{\mathrm{UF}-\mathrm{CMA}}(\lambda)=$ $\operatorname{Pr}\left[\operatorname{Exp}_{\mathcal{A}, \Sigma}^{\mathrm{UF}-\mathrm{CMA}}(\lambda)=1\right]$. Then we say that $\mathcal{A}(t, Q, \epsilon)$-breaks the UF-CMA-security of $\Sigma$ if $\mathcal{A}$ runs in time $t$, makes at most $Q$ signature queries, and $\operatorname{Adv}_{\mathcal{A}, \Sigma}^{\mathrm{UF}-\mathrm{CMA}}(\lambda)=\epsilon$.

A digital signature scheme $\Sigma$ is UF-CMA-secure if for any $\operatorname{PPT} \mathcal{A}, \operatorname{Ad} \mathbf{v}_{\mathcal{A}, \Sigma}^{\mathrm{UF}-\mathrm{CMA}}(\lambda)$ is negligible.

\section{Appendix B: On the hardness of the FDHI assumption}

To gain confidence in the FDHI assumption we show that FDHI is implied by the following decisional assumption:

Definition 12 (Decisional Assumption 1) Let $\mathcal{G}$ be a generator of asymmetric bilinear groups, let bgp $=\left(p, \mathbb{G}_{1}, \mathbb{G}_{2}, \mathbb{G}_{T}, g_{1}, g_{2}, e\right) \stackrel{\$}{\leftarrow}\left(1^{\lambda}\right)$ where $g_{1}, g_{2}$ are two random generators. The Decisional Assumption 1 is $\epsilon$-hard for $\mathcal{G}$ if for every PPT adversary $\mathcal{A}$ :

$$
\left|\operatorname{Pr}\left[\mathcal{A}\left(g_{1}, g_{2}, g_{2}^{z}, g_{2}^{v}, g_{1}^{\frac{z}{v}}, g_{1}^{r}, g_{1}^{\frac{r}{v}}, g_{2}^{1 / z}\right)\right]-\operatorname{Pr}\left[\mathcal{A}\left(g_{1}, g_{2}, g_{2}^{z}, g_{2}^{v}, g_{1}^{\frac{z}{v}}, g_{1}^{r}, g_{1}^{\frac{r}{v}}, g_{2}^{t}\right)\right]\right| \leq \epsilon
$$

where $z, v, r, t \stackrel{\$}{\leftarrow} \mathbb{Z}_{p}$.

Proposition 2 For any $\mathcal{A}$ which $\epsilon$-breaks the FDHI assumption, there is $\mathcal{B}$ which $\epsilon^{\prime}$-breaks Assumption 1 where $\epsilon^{\prime} \geq \epsilon-1 / p$

Proof (Sketch) Let $\left(g_{1}, g_{2}, g_{2}^{z}, g_{2}^{v}, g_{1}^{\frac{z}{v}}, g_{1}^{r}, g_{1}^{\frac{r}{v}}, T\right)$ be the input of $\mathcal{B}$ where $T$ can be either $g_{2}^{1 / z}$ or $g_{2}^{t}$ for a random and independent $t$. $\mathcal{B}$ runs $(W, Y) \leftarrow \mathcal{A}\left(g_{1}, g_{2}, g_{2}^{z}, g_{2}^{v}, g_{1}^{\frac{z}{v}}, g_{1}^{r}, g_{1}^{\frac{r}{v}}\right)$. If $e\left(Y, g_{2}^{Z}\right)=e\left(W, g_{2}\right)$ (i.e., $\mathcal{A}$ succeeds), then $\mathcal{B}$ returns 1 if $e(W, T)=e\left(Y, g_{2}\right)$ holds, and 0 otherwise.

Clearly, if $T=g_{2}^{1 / z}, e(W, T)=e\left(W, g_{2}^{1 / z}\right)=e\left(W^{1 / z}, g_{2}\right)=e\left(Y, g_{2}\right)$. Instead, if $T$ is random and independent, the equation holds only with negligible probability $1 / p$.

As a next step, we show that Assumption 1 can be equivalently re-written in the following Assumption 2 without rational exponents:

Definition 13 (Decisional Assumption 2) Let $\mathcal{G}$ be a generator of asymmetric bilinear groups, let bgp $=\left(p, \mathbb{G}_{1}, \mathbb{G}_{2}, \mathbb{G}_{T}, g_{1}, g_{2}, e\right) \stackrel{\$}{\leftarrow} \mathcal{G}\left(1^{\lambda}\right)$. Let $h_{1} \in \mathbb{G}_{1}, h_{2} \in \mathbb{G}_{2}$ be two random generators. The Decisional Assumption 2 is $\epsilon$-hard for $\mathcal{G}$ if for every PPT adversary $\mathcal{A}$ :

$$
\left|\operatorname{Pr}\left[\mathcal{A}\left(h_{1}, h_{2}, h_{2}^{x}, h_{2}^{u}, h_{1}^{u}, h_{1}^{r u}, h_{1}^{r x}, h_{2}^{x^{2}}\right)\right]-\operatorname{Pr}\left[\mathcal{A}\left(h_{1}, h_{2}, h_{2}^{x}, h_{2}^{u}, h_{1}^{u}, h_{1}^{r u}, h_{1}^{r x}, h_{2}^{t}\right)\right]\right| \leq \epsilon
$$

where $x, u, r, t \stackrel{\$}{\leftarrow} \mathbb{Z}_{p}$. 
Proof The equivalence between the assumptions is obtained by setting the following equalities:

$$
g_{1}=h_{1}^{u}, g_{2}=h_{2}^{x}, g_{2}^{z}=h_{2}, g_{2}^{v}=h_{2}^{u}, g_{1}^{z / v}=h_{1}, g_{1}^{r}=h_{1}^{r u}, g_{1}^{r / v}=h_{1}^{r x}, T=T
$$

Finally, it is not hard to see that Assumption 2 is hard in the generic bilinear group model. When framing the assumption according to the master theorem in [13], the polynomial $x^{2}$ (in the group $\mathbb{G}_{2}$ ) is in fact linearly-independent from the other polynomials representing the instance of the assumption. To confirm the validity of Assumption 2, we also automatically tested it using the generic group tool of [7]. ${ }^{7}$

\section{Appendix C: Programmable hash functions [30,31]}

Let $\mathbb{G}$ be a cyclic group and $\lambda \in \mathbb{N}$ be a security parameter. A group hash function $H$ with input length $\ell=\ell(\lambda)$ is defined by a couple of PPT algorithms $H=$ (PHF.Gen, PHF.Eval). Given the security parameter $\lambda$, PHF.Gen outputs a key $K \stackrel{\$}{\leftarrow}$ PHF.Gen $\left(1^{\lambda}\right)$ which is used for deterministically evaluate $H$ as $y \leftarrow \operatorname{PHF}$. Eval $(K, X) \in \mathbb{G}$, for any $x \in\{0,1\}^{\ell}$. We write $H(X)=$ PHF.Eval $(K, X)$.

A group hash function $H$ is an $(m, n, \gamma, \delta)$-programmable hash function if there exist two PPT algorithms PHF.TrapGen and PHF.TrapEval such that:

Syntactics:

Correctness:

Statistically-close trapdoor keys: For all generators $g, h \in \mathbb{G}$ and for $K \stackrel{\$}{\leftarrow}$ PHF.Gen $\left(1^{\lambda}\right)$ and $\left(K^{\prime}, t\right) \stackrel{\$}{\$}$ PHF. TrapGen $\left(1^{\lambda}, g, h\right)$, the keys $K$ and $K^{\prime}$ are statistically $\gamma$-close: $K \equiv^{\gamma} K$.

Well distributed logarithms: $\quad$ For all generators $g, h \in \mathbb{G}$ and all possible $K^{\prime}$ in the range of (the first component of ) PHF.TrapGen $\left(1^{\lambda}, g, h\right)$, for all $X_{1}, \ldots, X_{m}, Z_{1}, \ldots, Z_{n} \in\{0,1\}^{\ell}$ such that $X i \neq Z_{j}$ for any $i, j$, and for the corresponding $\left(a_{X_{i}}, b_{X_{i}}\right) \leftarrow$ PHF.TrapEval $\left(t, X_{i}\right)$ and $\left(a_{Z_{i}}, b_{Z_{i}}\right) \leftarrow$ PHF.TrapEval $\left(t, Z_{i}\right)$, we have

$$
\operatorname{Pr}\left[a_{X_{1}}=\cdots=a_{X_{m}}=0 \wedge a_{Z_{1}}, \ldots, a_{Z_{n}} \neq 0\right] \geq \delta
$$

where the probability is over the trapdoor $t$ that was produced along with $K^{\prime}$.

7 The simple script describing the assumption is available upon request. 


\section{References}

1. Abe M., Groth J., Ohkubo M., Tibouchi M.: Structure-preserving signatures from type II pairings. In: Garay J.A., Gennaro R. (eds.) CRYPTO 2014, Part I. LNCS, vol. 8616, pp. 390-407. Springer (2014).

2. Ahn J.H., Boneh D., Camenisch J., Hohenberger S., Shelat A., Waters B.: Computing on authenticated data. In: Cramer R. (ed.) TCC 2012. LNCS, vol. 7194, pp. 1-20. Springer (2012).

3. Attrapadung N., Libert B.: Homomorphic network coding signatures in the standard model. In: Catalano D., Fazio N., Gennaro R., Nicolosi A. (eds.) PKC 2011. LNCS, vol. 6571, pp. 17-34. Springer (2011).

4. Attrapadung N., Libert B., Peters T.: Computing on authenticated data: new privacy definitions and constructions. In: Wang X., Sako K. (eds.) ASIACRYPT 2012. LNCS, vol. 7658, pp. 367-385. Springer (2012).

5. Attrapadung N., Libert B., Peters T.: Efficient completely context-hiding quotable and linearly homomorphic signatures. In: Kurosawa K., Hanaoka G. (eds.) PKC 2013. LNCS, vol. 7778, pp. 386-404. Springer (2013).

6. Backes M., Fiore D., Reischuk R.M.: Verifiable delegation of computation on outsourced data. In: Sadeghi A.-R., Gligor V.D., Yung M. (eds.) ACM CCS 13, pp. 863-874. ACM Press (2013).

7. Barthe G., Fagerholm E., Fiore D., Mitchell J.C., Scedrov A., Schmidt B.: Automated analysis of cryptographic assumptions in generic group models. In: Garay J.A., Gennaro R. (eds.) CRYPTO 2014, Part I. LNCS, vol. 8616, pp. 95-112. Springer (2014).

8. Boneh D., Franklin M.K.: Identity-based encryption from the Weil pairing. In: Kilian J. (ed.) CRYPTO 2001. LNCS, vol. 2139, pp. 213-229. Springer (2001).

9. Boneh D., Boyen X.: Efficient selective-ID secure identity based encryption without random oracles. In: Cachin C., Camenisch J., (eds.) EUROCRYPT 2004. LNCS, vol. 3027, pp. 223-238. Springer (2004).

10. Boneh D., Boyen X.: Short signatures without random oracles. In: Cachin C., Camenisch J. (eds). EUROCRYPT 2004. LNCS, vol. 3027, pp. 56-73. Springer (2004).

11. Boneh D., Freeman D.M.: Homomorphic signatures for polynomial functions. In: Paterson K.G. (ed.) EUROCRYPT 2011. LNCS, vol. 6632, pp. 149-168. Springer (2011).

12. Boneh D., Freeman D.M.: Linearly homomorphic signatures over binary fields and new tools for latticebased signatures. In: Catalano D., Fazio N., Gennaro R., Nicolosi A. (eds.) PKC 2011. LNCS, vol. 6571, pp. 1-16. Springer (2011).

13. Boneh D., Boyen X., Goh E.-J.: Hierarchical identity based encryption with constant size ciphertext. In: Cramer R. (ed.) EUROCRYPT 2005. LNCS, vol. 3494, pp. 440-456. Springer (2005).

14. Boneh D., Freeman D., Katz J., Waters B.: Signing a linear subspace: signature schemes for network coding. In: Jarecki S., Tsudik G. (eds.) PKC 2009. LNCS, vol. 5443, pp. 68-87. Springer (2009).

15. Boyen X., Fan X., Shi E.: Adaptively secure fully homomorphic signatures based on lattices. Cryptology ePrint Archive, Report 2014/916. http://eprint.iacr.org/2014/916 (2014).

16. Catalano D., Fiore D., Warinschi B.: Adaptive pseudo-free groups and applications. In: Paterson K.G. (ed.) EUROCRYPT 2011. LNCS, vol. 6632, pp. 207-223. Springer (2011).

17. Catalano D., Fiore D., Warinschi B.: Efficient network coding signatures in the standard model. In: Fischlin M., Buchmann J., Manulis M. (eds.) PKC 2012. LNCS, vol. 7293, pp. 680-696. Springer (2012).

18. Catalano D., Fiore D., Gennaro R., Vamvourellis K.: Algebraic (trapdoor) one-way functions and their applications. In: Sahai A. (ed.) TCC 2013. LNCS, vol. 7785, pp. 680-699. Springer (2013).

19. Catalano D., Fiore D., Nizzardo L.: Programmable hash functions go private: constructions and applications to (homomorphic) signatures with shorter public keys. In: CRYPTO 2015. Springer (2015).

20. Catalano D., Fiore D., Warinschi B.: Homomorphic signatures with efficient verification for polynomial functions. In: Garay J.A., Gennaro R. (eds.) CRYPTO 2014, Part I. LNCS, vol. 8616, pp. 371-389. Springer (2014).

21. Erdös P., Frankel P., Furedi Z.: Families of finite sets in which no set is covered by the union of $r$ others. Isr. J. Math. 51, 79-89 (1985).

22. Freeman D.M.: Improved security for linearly homomorphic signatures: a generic framework. In: Fischlin M., Buchmann J., Manulis M. (eds.) PKC 2012. LNCS, vol. 7293, pp. 697-714. Springer (2012).

23. Freire E.S.V., Hofheinz D., Paterson K.G., Striecks C.: Programmable hash functions in the multilinear setting. In: Canetti R., Garay J.A. (eds.) CRYPTO 2013, Part I. LNCS, vol. 8042, pp. 513-530. Springer (2013).

24. Gennaro R., Katz J., Krawczyk H., Rabin T.: Secure network coding over the integers. In: Nguyen P.Q., Pointcheval D. (eds.) PKC 2010. LNCS, vol. 6056, pp. 142-160. Springer (2010).

25. Gennaro R., Wichs D.: Fully homomorphic message authenticators. In: Sako K., Sarkar P. (eds.) ASIACRYPT 2013, Part II. LNCS, vol. 8270, pp. 301-320. Springer (2013).

26. Gorbunov S., Vaikuntanathan V., Wichs D.: Leveled fully homomorphic signatures from standard lattices. In: 47th ACM STOC. ACM Press (2015). 
27. Green M., Hohenberger S.: Practical adaptive oblivious transfer from simple assumptions. In: Ishai Y. (ed.) TCC 2011. LNCS, vol. 6597, pp. 347-363. Springer (2011).

28. Hanaoka G., Matsuda T., Schuldt J.C.N.: On the impossibility of constructing efficient key encapsulation and programmable hash functions in prime order groups. In: Safavi-Naini R., Canetti R. (eds.) CRYPTO 2012. LNCS, vol. 7417, pp. 812-831. Springer (. 2012).

29. Haralambiev K., Jager T., Kiltz E., Shoup V.: Simple and efficient public-key encryption from computational Diffie-Hellman in the standard model. In: Nguyen P.Q., Pointcheval D. (eds.) PKC 2010. LNCS, vol. 6056, pp. 1-18. Springer (2010).

30. Hofheinz D., Kiltz E.: Programmable hash functions and their applications. In: Wagner D. (ed.) CRYPTO 2008. LNCS, vol. 5157, pp. 21-38. Springer (2008).

31. Hofheinz D., Kiltz E.: Programmable hash functions and their applications. J. Cryptol. 25(3), 484-527 (2012).

32. Hofheinz D., Jager T., Kiltz E.: Short signatures from weaker assumptions. In: Lee D.H., Wang X. (eds.) ASIACRYPT 2011. LNCS, vol. 7073, pp. 647-666. Springer (2011).

33. Johnson R., Molnar D., Song D.X., Wagner D.: Homomorphic signature schemes. In: Preneel B. (ed.) CT-RSA 2002. LNCS, vol. 2271, pp. 244-262. Springer (2002).

34. Kumar R., Rajagopalan S., Sahai A.: Coding constructions for blacklisting problems without computational assumptions. In: Wiener M.J. (ed.) CRYPTO'99. LNCS, vol. 1666, pp. 609-623. Springer (1999).

35. Libert B., Peters T., Joye M., Yung M.: Linearly homomorphic structure-preserving signatures and their applications. In: Canetti R., Garay J.A. (eds.) CRYPTO 2013, Part II. LNCS, vol. 8043, pp. 289-307. Springer (2013).

36. Mitsunari S., Saka R., Kasahara M.: A new traitor tracing. IEICE Trans. E85-A(2), 481-484 (2002).

37. Schwartz J.T.: Fast probabilistic algorithms for verification of polynomial identities. J. ACM 27, 701-717 (1980).

38. Waters B.R.: Efficient identity-based encryption without random oracles. In: Cramer R. (ed.) EUROCRYPT 2005. LNCS, vol. 3494, pp. 114-127. Springer (2005).

39. Yamada S., Hanaoka G., Kunihiro N.: Two-dimensional representation of cover free families and its applications: short signatures and more. In: Dunkelman O. (ed.) CT-RSA 2012. LNCS, vol. 7178, pp. 260-277. Springer (2012).

40. Zippel R.: Probabilistic algorithms for sparse polynomials. In: Ng E.W. (ed.) EUROSM '79. Lecture Notes in Computer Science, vol. 72, pp. 216-226. Springer (1979).

41. Zhang J., Chen Y., Zhang Z.: Programmable hash functions from lattices: Short signatures and IBEs with small key sizes. In: Robshaw M., Katz J. (eds.) Advances in Cryptology—CRYPTO 2016. Lecture Notes in Computer Science, vol. 9816. Springer, Berlin (2016). 